\title{
TOROIDAL AND REDUCTIVE BOREL-SERRE COMPACTIFICATIONS OF LOCALLY SYMMETRIC SPACES
}

\author{
By Mark Goresky and Yung-Sheng TAI
}

\begin{abstract}
By "Hermitian locally symmetric space" we mean an arithmetic quotient of a bounded symmetric domain. Both the toroidal and the reductive Borel-Serre compactifications of such a space come equipped with canonical mappings to the Baily-Borel Satake compactification. In this article we show that there is a mapping from the toroidal compactification to the reductive Borel-Serre compactification, whose composition with the projection to the Baily-Borel compactification agrees with the canonical projection up to an arbitrarily small homotopy. We also consider arithmetic quotients of a self-adjoint homogeneous cone. There is a canonical mapping from the reductive Borel-Serre compactification to the standard compactification of such a locally symmetric cone. We show that this projection, when restricted to the closure of a polyhedral cone, has contractible fibers.
\end{abstract}

Introduction. During the last twenty years, several topological approaches have been developed in order to study the action of Hecke operators on the cohomology of arithmetic groups and of Shimura varieties. Let $X=\Gamma \backslash G / K$ be a Hermitian locally symmetric space (where $G=\mathbf{G}(\mathbb{R})$ are the real points of a semisimple algebraic group $\mathbf{G}$ defined over $\mathbb{Q}, K \subset G$ is a maximal compact subgroup, $\Gamma \subset \mathbf{G}(\mathbb{Q})$ is a neat arithmetic subgroup, and $G / K$ is assumed to carry a $G$-invariant complex structure). A central object of study is the BailyBorel Satake compactification $\bar{X}^{B B}$ : it is a complex algebraic variety which may be highly singular. One approach to understanding the topology of this space is through the toroidal compactification $g: \bar{X}_{\Sigma}^{t o r} \rightarrow \bar{X}^{B B}$ of [AMRT]. It is not unique but involves a choice $\Sigma$ of $\Gamma$-equivariant polyhedral cone decomposition of certain self adjoint homogeneous cones; if $\Sigma$ is chosen sufficiently fine then the toroidal compactification is a resolution of singularities of $\bar{X}^{B B}$. Essential use of this resolution was made, for example, by E. Looijenga [L] in his proof of the Zucker conjecture.

A second approach to understanding the Baily-Borel compactification involves the reductive Borel-Serre compactification ([Z1] $\S 4$ ), $\mu: \bar{X}^{R B S} \rightarrow \bar{X}^{B B}$. This compactification is neither complex algebraic, nor is it smooth. Nevertheless, its singularities are easily understood. In a series of papers ([GM1], [GM2], [GHM], [GKM]) it was shown that Arthur's $L^{2}$ Lefschetz formula for Hecke correspondences on $X$ may be interpreted term for term as the Lefschetz fixed point formula for the weighted cohomology of $\bar{X}^{R B S}$. One is therefore led to

Manuscript received December 9, 1997; revised December 23, 1998.

Research of the first author supported in part by NSF Grants \#DMS9304580 and \#DMS9626616.

American Journal of Mathematics 121 (1999), 1095-1151. 
the problem of comparing these two "resolutions" of $\bar{X}^{B B}$. In [Ji], L. Ji showed that there is little hope in comparing these two compactifications, because their greatest common quotient is the Baily-Borel compactification. (Ji's paper was motivated by the paper [HZ] of M. Harris and S. Zucker in which they conjectured that the Baily-Borel compactification is also the greatest common quotient of the Borel-Serre compactification and the toroidal compactification. In the same article [Ji], Ji determined the G.C.Q. of the Borel-Serre and toroidal compactifications and completely determined the conditions under which it coincides with the Baily-Borel compactification.)

In this paper we revive hopes for comparing the two compactifications $\bar{X}^{R B S}$ and $\bar{X}_{\Sigma}^{\text {tor }}$. Let $\hat{X} \subset \bar{X}^{R B S} \times \bar{X}_{\Sigma}^{\text {tor }}$ denote the closure of the diagonal embedding of $X$ in these two compactifications. Let $\theta_{1}$ and $\theta_{2}$ denote the projections to the first and second factor. In $\S 7.2$ we prove the following:

THEOREM A. If the polyhedral decomposition $\Sigma$ is chosen sufficiently fine then the fibers of the projection $\theta_{2}: \hat{X} \rightarrow \bar{X}_{\Sigma}^{\text {tor }}$ are contractible.

In $§ 7.3$ we conclude:

CoRollary. The resolution $g: \bar{X}_{\Sigma}^{t o r} \rightarrow \bar{X}^{B B}$ is homotopic to a mapping $g^{\prime}$ which factors through $\bar{X}^{R B S}$.

It follows, for example, that the compact support cohomology of $X$, and the cohomology of $X$ and its compactifications are all related in a single sequence of compatible homomorphisms,

$$
H_{c}^{*}(X) \rightarrow H^{*}\left(\bar{X}^{B B}\right) \rightarrow H^{*}\left(\bar{X}^{R B S}\right) \rightarrow H^{*}\left(\bar{X}_{\Sigma}^{t o r}\right) \rightarrow H^{*}(X)
$$

Further applications to automorphic vectorbundles are described in $\S 9$.

The mapping $g^{\prime}: \bar{X}_{\Sigma}^{t o r} \rightarrow \bar{X}_{B B}$ is obtained as the composition

$$
\bar{X}_{\Sigma}^{\text {tor }} \stackrel{\tau}{\longrightarrow} \hat{X} \stackrel{\theta_{1}}{\longrightarrow} \bar{X}^{R B S} \stackrel{\mu}{\longrightarrow} \bar{X}^{B B}
$$

where $\tau$ is a homotopy inverse to $\theta_{2}$. In $\oint 8$ we show that the homotopy inverse $\tau$ may be chosen to be the identity on the complement of an arbitrarily small regular neighborhood of the boundary. Although it is not possible to find a homotopy inverse $\tau$ so that $\theta_{2} \tau$ is the identity, it is possible to guarantee that, for each stratum $S$ of $\bar{X}^{t o r}, \theta_{2} \tau(\bar{S})=\bar{S}$, and hence $g(\bar{S})=g^{\prime}(\bar{S})$.

Theorem A is complementary to the closely related results of $\mathrm{M}$. Harris and S. Zucker [HZ]. Theorem A was conjectured by R. MacPherson and M. Rapoport [R] in 1991 and was verified by them in the case that the rational rank of $\mathbf{G}$ is 1 . In this case, if a point $x \in \bar{X}_{\Sigma}^{\text {tor }}$ corresponds to a (closed) polyhedral cone $\sigma \in \Sigma$, 
then the fiber $\theta_{2}^{-1}(x)$ is not only contractible but it may be canonically identified with the quotient $[\sigma]$ of $\sigma-\{0\}$ under homotheties, i.e., it is homeomorphic to a convex polyhedron. The case of higher rank turned out to be much more difficult than we expected: the fiber $\theta_{2}^{-1}(x)$ is obtained from the convex polyhedron $[\sigma]$ by a sequence of "real" blowups.

There are three main steps in the proof of Theorem A. First, suppose that $C \subset$ $V$ is a (rationally defined) self-adjoint open homogeneous cone in some rationally defined real vectorspace $V$, and suppose that $\sigma \subset V$ is a rational polyhedral cone whose interior $\sigma^{o} \subset C$ is contained in $C$. Let $\left[\sigma^{o}\right] \subset[C] \subset \mathbb{P}(V)$ denote the quotient under homotheties. Theorem B (§2.8) states that the closure (which we denote by $[\sigma]^{R B S}$ ) of $\left[\sigma^{o}\right]$ in the reductive Borel-Serre partial compactification $\overline{[C]}^{R B S}$ of $[C]$ is contractible. In fact, this closure is a "blowup" of the convex polyhedron $[\sigma]$. The fibers of the projection $[\sigma]^{R B S} \rightarrow[\sigma]$ are again sets of the form $[\tau]^{R B S}$. (This, in turn, is a consequence of Lemma 2.6.2 which describes the relationship between convergence in $\overline{[C]}^{R B S}$ and convergence in the "standard" Satake compactification $\bar{C}^{\text {std }}$ of $C$.)

Theorem B was proven by $\mathrm{M}$. McConnell [Mc] in the case that $C$ is the self adjoint cone of positive definite $3 \times 3$ matrices; in fact, he shows that the set $[\sigma]^{R B S}$ is homeomorphic to a convex polyhedron, and that the same is true for the closure of $[\sigma]$ in any Satake compactification of $[C]$. It seems possible that this result may hold in general, i.e., for any rationally defined self adjoint homogeneous cone $C$ and for any rational polyhedral cone $\sigma \subset C$, the closure $[\sigma]^{S}$ of $\left[\sigma^{o}\right]$ in any Satake partial compactification $\overline{[C]}^{S}$ may be homeomorphic to a convex polyhedron.

The second step in the proof of Theorem A is to guarantee that the appropriate discrete group $\Gamma_{\ell}$ does not introduce any identifications on $[\sigma]^{R B S}$ under the projection $\overline{[C]}^{R B S} \rightarrow \Gamma_{\ell} \backslash \overline{[C]}^{R B S}$. This may be accomplished by assuming that the polyhedral cone $\sigma$ is sufficiently small $(\S 3)$. We show that any $\Gamma_{\ell^{-}}$-equivariant admissible rational polyhedral decomposition $\Sigma$ of $C$ admits a $\Gamma_{\ell}$-equivariant admissible refinement, each of whose cones is sufficiently small. The existence of equivariant refinements of polyhedral cone decompositions is not very carefully treated in [AMRT], so we quickly review the relevant techniques in $\S 3$.

Finally we show that the set $[\sigma]^{R B S}$ appears as the fiber of the projection $\theta_{2}: \hat{X} \rightarrow \bar{X}_{\Sigma}^{t o r}$. In order to prove this, one is forced to relate convergence in $\bar{X}^{R B S}$ with the linear structure of the polyhedral cone $\sigma \subset V$, which is accomplished in Theorem $C$ ( $\S 4.2)$. The proof turns out to involve some delicate estimates $(\S 5,6)$ involving the explicit descriptions of the roots of $G$.

Acknowledgments. We are happy to thank R. MacPherson and M. Rapoport for useful conversations. We have also profited from discussions with A. Borel and L. Ji. We are especially grateful to the referee and to S. Zucker (if separate thanks be needed) for a series of very helpful comments which have considerably improved this paper. We would like to thank Dale Husemoller for bringing us 
together. The first author would like to thank the Institute for Advanced Study in Princeton, NJ, for its hospitality and support, as well as the Ellentuck Foundation and the National Science Foundation.

1. Symmetric spaces. In this section we review some basic results of Borel and Serre $[\mathrm{BS}]$ and establish the notation which we will use throughout this paper.

1.1. Parabolic subgroups. Algebraic groups will be designated by boldface type $(\mathbf{G}, \mathbf{P}$, etc.). If an algebraic group is defined over the real numbers then its group of real points will be in Roman $(G=\mathbf{G}(\mathbb{R}), P=\mathbf{P}(\mathbb{R})$, etc.). The connected component of the identity is denoted with a superscript $0\left(\mathbf{G}^{0}, \mathbf{P}^{0}\right.$, etc. $)$. If $\mathbf{S}$ is an algebraic torus then the identity component of the group of real points will be denoted by $A=\mathbf{S}(\mathbb{R})^{0}$, and may be inaccurately referred to as a torus. If $\mathbf{G}$ is a reductive algebraic group which is defined over the rational numbers $\mathbb{Q}$, we denote by $\mathbf{S}_{\mathbf{G}}$ the greatest $\mathbb{Q}$-split torus in the center of $\mathbf{G}$, and set $A_{G}=\mathbf{S}_{\mathbf{G}}(\mathbb{R})^{0}$. Then the group of real points splits as a direct product

$$
G=A_{G} \times{ }^{0} \mathbf{G}(\mathbb{R})
$$

where

$$
{ }^{0} \mathbf{G}=\bigcap_{\chi} \operatorname{ker}\left(\chi^{2}\right)
$$

denotes the intersection of the kernels of all the algebraically defined rational characters $\chi \in \operatorname{Mor}\left(\mathbf{G}, G L_{1}\right)$. The group ${ }^{0} G \subset G$ contains all compact and arithmetic subgroups of $G$.

For any parabolic subgroup $\mathbf{P} \subset \mathbf{G}$ we denote by $\mathcal{U}_{\mathbf{P}}$ the unipotent radical of $\mathbf{P}$, and by $\nu_{P}: \mathbf{P} \rightarrow \mathbf{L}_{\mathbf{P}}=\mathbf{P} / \mathcal{U}_{\mathbf{P}}$ the projection to the Levi quotient. We denote by

$$
M_{P}={ }^{0} L_{P}
$$

If $\mathbf{S}_{\mathbf{P}} \subset \mathbf{L}_{\mathbf{P}}$ denotes the greatest $\mathbb{Q}$-split torus in the center of $\mathbf{L}_{\mathbf{P}}$ then $L_{P}=A_{P} M_{P}$ splits as a (commuting) direct product. Any lift $i: \mathbf{L}_{\mathbf{P}} \rightarrow \mathbf{P}$ of $\mathbf{L}_{\mathbf{P}}$ determines a Langlands decomposition (which is a semi-direct product),

$$
P=\mathcal{U}_{P} \cdot i\left(A_{P} M_{P}\right)
$$

Choose a minimal rational parabolic subgroup $\mathbf{Q}_{0} \subset \mathbf{G}$ and call it standard. Choose a rationally defined lift $i: \mathbf{L}_{\mathbf{Q}_{0}} \rightarrow \mathbf{Q}_{0}$ and let $\mathbf{S}=i\left(\mathbf{S}_{\mathbf{Q}_{0}}\right)$ be the resulting lift of the greatest $\mathbb{Q}$-split torus in the center of $\mathbf{L}\left(\mathbf{Q}_{0}\right)$, so that $\mathbf{S} \subset \mathbf{Q}_{0} \subset \mathbf{G}$. Then $\mathbf{S}$ is a maximal $\mathbb{Q}$-split torus in $\mathbf{G}$. The root system $\Phi(\mathbf{S}, \mathbf{G})$ admits a linear order so that the positive roots $\Phi^{+}(\mathbf{S}, \mathbf{G})$ are those occurring in $\mathcal{U}_{\mathbf{P}}$. Let $\Delta=\Delta(\mathbf{S}, \mathbf{G})$ 
denote the resulting set of simple positive roots. The elements $\phi \in \Delta$ are trivial on $\mathbf{S}_{G}$ and form a basis for the character module $\chi\left(\mathbf{S} / \mathbf{S}_{G}\right) \otimes_{\mathbb{Z}} \mathbb{Q}$.

The rational parabolic subgroups which contain $\mathbf{Q}_{0}$ are in one-to-one correspondence with subsets $I \subset \Delta$. For a given subset $I \subset \Delta$ define

$$
\mathbf{S}(I)=\left(\bigcap_{\phi \in I} \operatorname{ker}(\phi)\right)^{0}
$$

If $Z$, then the corresponding parabolic subgroup is

$$
\mathbf{P}=\mathbf{P}(I)=Z(\mathbf{S}(I)) \mathcal{U}_{\mathbf{Q}_{0}}=Z(\mathbf{S}(I)) \mathcal{U}_{\mathbf{P}}
$$

(the latter decomposition being a semidirect product). Then $\mathbf{S}(I)=\mathbf{S}_{P(I)}$ and $Z(\mathbf{S}(I))$ is a lift of the Levi quotient $\mathbf{L}_{\mathbf{P}(I)}$. We denote by

$$
\Delta_{P}=\{\phi \mid \mathbf{S}(I)\}_{\phi \in \Delta-I}
$$

the restrictions of the remaining simple roots to the torus $\mathbf{S}(I)$; they form a rational basis for the character module $\chi\left(\mathbf{S}(I) / \mathbf{S}_{G}\right) \otimes \mathbb{Q}$. If $I \subset J \subset \Delta$ then $\mathbf{S}(I) \supset \mathbf{S}(J)$ and $\mathbf{P}(I) \subset \mathbf{P}(J)$.

Let $K \subset G$ be a maximal compact subgroup and define $D=G / K$. The space $D$ is referred to as a "generalized symmetric space." If $\mathbf{S}_{G}$ is not trivial, then we denote by $[D]=G / K A_{G}$ the quotient of $D$ under the identity component of this central torus. There is a unique basepoint $x_{0} \in D$ with $K=\operatorname{Stab}_{G}\left(x_{0}\right)$. This choice also determines the following data:

(1) A maximal compact subgroup $K_{P}=K_{P}\left(x_{0}\right)=K \cap P$ and a diffeomorphism $P / K_{P} \rightarrow D$.

(2) A Cartan involution $\theta: G \rightarrow G$ with $G^{\theta}=K$.

(3) A unique lifting $i_{x_{0}}: \mathbf{L}_{\mathbf{P}} \rightarrow \mathbf{P}$ of the Levi quotient whose image $L_{P}\left(x_{0}\right)$ is $\theta$-stable. For any subset $B \subseteq L_{P}$ we denote its lift by $B\left(x_{0}\right)=i_{x_{0}}(B)$. The basepoint $x_{0} \in D$ is rational for $P$ if the $\operatorname{lift} \mathbf{L}_{\mathbf{P}}\left(x_{0}\right) \subset \mathbf{G}$ is a rationally defined algebraic subgroup.

(4) A diffeomorphism

$$
D_{P}=P / K_{P} A_{P} \mathcal{U}_{P} \cong M_{P} / K_{P}
$$

given by $m K_{P} \mapsto i_{x_{0}}(m) K_{P} A_{P} \mathcal{U}_{P}$.

(5) A canonical rational Langlands' decomposition

$$
P=\mathcal{U}_{P} A_{P}\left(x_{0}\right) M_{P}\left(x_{0}\right) .
$$


(6) A diffeomorphism

$$
\mathcal{U}_{P} \times A_{P} \times D_{P} \rightarrow D
$$

given by $\left(u, a, m K_{P}\right) \rightarrow u i_{x_{0}}(a) i_{x_{0}}(m) K_{P}\left(x_{0}\right)$ (where $\left.m K_{P} \in M_{P} / K_{P} \cong D_{P}\right)$.

(7) Projections $u_{P}: D \rightarrow \mathcal{U}_{P}, \alpha_{P}: D \rightarrow A_{P}$ and $\Phi_{P}: D \rightarrow D_{P}$ to the first, second, and third factors. (The projection $\Phi_{P}$ is actually independent of the basepoint.)

In the coordinates given by (1.1.10), the action of $g \in P$ on $D$ and the geodesic action (see below) of $b \in A_{P}$ on $D$ are given by

$$
g\left(u, a, m K_{P}\right) \bullet b=\left(g u i_{x_{0}} \nu_{P}\left(g^{-1}\right), a b c, x m K_{P}\right)
$$

where $\nu_{P}(g)=c x \in A_{P} M_{P}=L_{P}$.

The canonical Langlands decompositions (1.1.9) of two parabolic subgroups $\mathbf{Q} \subset \mathbf{P}$ are related in the following way. The image $\bar{Q}=\nu_{P}(Q) \subset L_{P}$ is parabolic in $L_{P}$. Set $\mathcal{U}_{\bar{Q}}\left(x_{0}\right)=i_{x_{0}}\left(\mathcal{U}_{\bar{Q}}\right)$ and $A^{\prime}\left(x_{0}\right)=i_{x_{0}}\left(A_{Q}\right) \cap M_{P}\left(x_{0}\right)$. Then

$$
\begin{aligned}
Q & =\left(\mathcal{U}_{P} \mathcal{U}_{\bar{Q}}\left(x_{0}\right)\right)\left(A_{P}\left(x_{0}\right) A^{\prime}\left(x_{0}\right)\right) M_{Q}\left(x_{0}\right) \\
& =\mathcal{U}_{P} A_{P}\left(x_{0}\right)\left(\mathcal{U}_{\bar{Q}}\left(x_{0}\right) A^{\prime}\left(x_{0}\right) M_{Q}\left(x_{0}\right)\right) .
\end{aligned}
$$

The first is the canonical Langlands decomposition of $Q$ while the second is the decomposition of $Q$ which is induced from the canonical Langlands decomposition of $P$.

1.2. Borel-Serre partial compactification. Throughout this section we suppose that $\mathbf{G}$ is a reductive algebraic group defined over $\mathbb{Q}$ with $\mathbf{S}_{G}=\{1\}$. Fix a basepoint $x_{0} \in D$ in the associated generalized symmetric space, with stabilizer $K=K\left(x_{0}\right)=\operatorname{Stab}_{G}\left(x_{0}\right)$. Let $\mathbf{P} \subset \mathbf{G}$ be a rational parabolic subgroup. The torus $A_{P} \subset L_{P}$ acts on $D$ in two ways. Write $D=P / K_{P}$. The action from the left is given by $a \cdot g K_{P}:=i_{x_{0}}(a) g K_{P}$ (for any $g \in P$ ), while the action from the right (which is the geodesic action of Borel and Serre) is given by $\left(g K_{P}\right) \bullet a:=g i_{x_{0}}(a) K_{P}$. The geodesic action is well defined since $A_{P}\left(x_{0}\right)=i_{x_{0}}\left(A_{P}\right)$ commutes with $K_{P}=i_{x_{0}}\left(\nu_{P}\left(K_{P}\right)\right)$, and it is even independent of the choice of basepoint. The quotient $e_{P}=D / A_{P}$ is called the Borel-Serre boundary component or the Borel-Serre stratum corresponding to $P$.

The characters $\beta \in \Delta_{P}$ determine a diffeomorphism $A_{P} \cong\left(\mathbb{R}_{>0}\right)^{\Delta_{P}}$ and we denote by $\overline{A_{P}}$ the partial compactification obtained by adding the point at infinity to each copy of $\mathbb{R}_{>0}$, i.e.

$$
\overline{A_{P}} \cong(0, \infty]^{\Delta_{P}}
$$

Then the "corner" associated to $P$ is the (noncompact) smooth manifold with 
corners, $D(P)=D \times_{A_{P}} \overline{A_{P}}$; it is a disjoint union

$$
D(P)=D \cup \coprod_{P^{\prime} \supseteq P} e_{P^{\prime}}
$$

and it is an open neighborhood of $e_{P}$ in the Borel-Serre partial compactification $\bar{D}^{B S}$ of $D$. The canonical projection

$$
\theta_{P}: D(P) \rightarrow D / A_{P}=e_{P}
$$

is the unique continuous extension of the mapping $D=P / K_{P} \rightarrow P / K_{P} A_{P}=e_{P}$.

Each $\beta \in \Delta_{P}$ determines a root function $f_{\beta}^{P}: D \rightarrow \mathbb{R}_{>0}$ by $f_{\beta}^{P}(x)=\beta\left(\alpha_{P}(x)\right)$. In other words, write $x=\operatorname{uam} K_{P}$ by (1.1.9); then $f_{\beta}^{P}(x)=\beta(a)$. The root function $f_{\beta}^{P}$ is equivariant with respect to the $P$ action and the geodesic action on $D$ in the following sense: If $g^{\prime}=u^{\prime} a^{\prime} m^{\prime} \in \mathcal{U}_{P} A_{P}\left(x_{0}\right) M_{P}\left(x_{0}\right)$ and if $b^{\prime} \in A_{P}$ then

$$
f_{\beta}^{P}\left(g^{\prime} x \bullet b^{\prime}\right)=f_{\beta}^{P}(x) \beta\left(a^{\prime} b^{\prime}\right)
$$

which follows from (1.1.11). It follows that $f_{\beta}^{P}$ extends to a function (which we also denote in the same way),

$$
f_{\beta}^{P}: D(P) \rightarrow(0, \infty]
$$

hence the mapping

$$
D(P) \rightarrow e_{P} \times(0, \infty]^{\Delta_{P}}
$$

given by $x \mapsto\left(\theta_{P}(x),\left\{f_{\beta}^{P}(x)\right\}_{\beta \in \Delta_{P}}\right)$ is a diffeomorphism of manifolds with corners. The following lemma characterizes convergent sequences in the Borel-Serre partial compactification of $D$.

LeMma 1.2.7. Let $\mathbf{Q}_{0}$ be a minimal parabolic subgroup of $\mathbf{G}$ and let $\mathbf{P}=\mathbf{P}(\mathbf{I})$ be a standard parabolic subgroup corresponding to a subset I $\subset \Delta$. Let $x_{\infty} \in$ $e_{P}$. Then a sequence of points $\left\{x_{k}\right\} \subset D$ converges (in the Borel-Serre partial compactification) to $x_{\infty}$ iff the following two conditions hold:

(1) $\theta_{P}\left(x_{k}\right) \in e_{P}$ converges in $e_{P}$ to $x_{\infty}$

(2) $f_{\beta}^{Q_{0}}\left(x_{k}\right) \rightarrow \infty$ for all $\beta \in \Delta-I$.

Proof 1.2.8. Let us consider the convergence of the sequence $\left\{x_{k}\right\}$ in the open corner $D\left(Q_{0}\right)$. By (1.2.6) (with $P$ replaced by $Q_{0}$ ), we have a diffeomorphism $D\left(Q_{0}\right) \cong e_{Q_{0}} \times(0, \infty]^{\Delta}$ which takes $e_{P} \cong e_{Q_{0}} \times(0, \infty)^{I} \times\{\infty\}^{\Delta-I}$. So we need 
to show that

(1) $\theta_{Q_{0}}\left(x_{k}\right)$ converges to $\theta_{Q_{0}}\left(x_{\infty}\right)$,

(2) $f_{\alpha}^{Q_{0}}\left(x_{k}\right) \rightarrow f_{\alpha}^{Q_{0}}\left(x_{\infty}\right)$ for all $\alpha \in I$,

(3) $f_{\beta}^{Q_{0}}\left(x_{k}\right) \rightarrow \infty$ for all $\beta \in \Delta-I$.

Items (1) and (3) follow immediately from the hypotheses. Let us consider item (2). For any $a \in A_{P}=A_{P(I)}$ and for any $\alpha \in I \subset \Delta$ we have $f_{\alpha}^{Q_{0}}\left(x_{k} \bullet a\right)=$ $f_{\alpha}^{Q_{0}}\left(x_{k}\right) \cdot \alpha(a)=f_{\alpha}^{Q_{0}}\left(x_{k}\right)$ by (1.2.4) and (1.1.5). But $\theta_{P}\left(x_{k}\right)=x_{k}\left(\bmod A_{P}\right)$ so

$$
f_{\alpha}^{Q_{0}}\left(x_{k}\right)=f_{\alpha}^{Q_{0}}\left(\theta_{P}\left(x_{k}\right)\right) \rightarrow f_{\alpha}^{Q_{0}}\left(x_{\infty}\right)
$$

as claimed.

1.2.9. The action of $\mathbf{G}(\mathbb{Q})$ on $D$ extends continuously to the partial compactification $\bar{D}^{B S}$. If $\Gamma \subset \mathbf{G}(\mathbb{Q})$ is a neat arithmetic group then the quotient $\bar{X}^{B S}=\Gamma \backslash \bar{D}^{B S}$ is the Borel-Serre compactification of $X=\Gamma \backslash D$. It is a manifold with corners; the image of each boundary component $e_{P}$ is called a boundary stratum.

1.3. Reductive Borel-Serre partial compactification. If $\mathbf{P} \subset \mathbf{G}$ is a rational parabolic subgroup, define the reductive Borel-Serre boundary component $D_{P}=P / K_{P} A_{P} \mathcal{U}_{P} \cong M_{P} / K_{P}$. Let

$$
\Phi_{P}: D=P / K_{P} \rightarrow P / K_{P} A_{P} \mathcal{U}_{P}=D_{P}
$$

denote the projection. Then $D_{P}$ is a "generalized symmetric space" (for the group $\left.M_{P}\right)$ and it inherits a basepoint $\Phi_{P}\left(x_{0}\right)$. The reductive Borel-Serre partial compactification ([Z1] $\S 4) \bar{D}^{R B S}$ is obtained from the Borel-Serre partial compactification by collapsing the fibers of the projection $e_{P}=P / K_{P} A_{P} \rightarrow D_{P}=P / K_{P} A_{P} \mathcal{U}_{P}$ to points (for every proper rational parabolic subgroup $\mathbf{P} \subset \mathbf{G}$ ). It is a Hausdorff topological space. The closure of $D_{P}$ in $\bar{D}^{R B S}$ is the reductive Borel-Serre partial compactification ${\overline{D_{P}}}^{R B S}$ of $D_{P}=M_{P} / K_{P}$. The projections $e_{P} \rightarrow D_{P}$ fit together to give a $\mathbf{G}(\mathbb{Q})$ equivariant continuous surjection $\bar{D}^{B S} \rightarrow \bar{D}^{R B S}$ which extends the identity mapping on $D$. Denote by $D[P] \subset \bar{D}^{R B S}$ the image of the corner $D(P)$ under this surjection, so that $D[P]=D \cup \coprod_{Q \supseteq P} D_{Q}$. Then $D[P]$ is an open neighborhood in $\bar{D}^{R B S}$ of the stratum $D_{P}$.

Suppose $\mathbf{Q} \subset \mathbf{P}$ are standard rational parabolic subgroups corresponding to subset $J \subset I \subset \Delta$ of the simple rational roots, respectively. Zucker's vexatious point [Z1] (3.19) is that the root functions $f_{\beta}^{P}$ and $f_{\beta}^{Q}$ usually do not agree. Let $\overline{\mathbf{Q}}=\nu_{P}(\mathbf{Q}) \subset \mathbf{L}_{\mathbf{P}}$ be the parabolic subgroup of $\mathbf{L}_{\mathbf{P}}$ which is determined by $\mathbf{Q}$. For $x \in D$ use (1.1.12) to write $x=u$ wabm $\cdot x_{0}$ with $u \in \mathcal{U}_{P}, w \in i_{x_{0}}\left(\mathcal{U}_{\bar{Q}}\right), a \in A_{P}\left(x_{0}\right)$, 
$b \in A^{\prime}=A_{Q}\left(x_{0}\right) \cap M_{P}\left(x_{0}\right)$, and $m \in M_{Q}\left(x_{0}\right)$. Then for all $\beta \in \Delta$ we have

$$
f_{\beta}^{Q}(x)=\beta(a b), \text { and } f_{\beta}^{P}(x)=\beta(a)
$$

(but $\beta \in I \Longrightarrow \beta(a)=1$ while $\beta \in J \Longrightarrow \beta(a)=\beta(b)=1$; see also Lemma 1.3.6 $\left(2^{\prime}\right)$ ). Since $\Phi_{P}(x)=w b m \cdot \Phi_{P}\left(x_{0}\right) \in M_{P} / K_{P}$, we have (for all $\beta \in I$ ),

$$
f_{\beta}^{\bar{Q}}\left(\Phi_{P}(x)\right)=\beta(b)=f_{\beta}^{Q}(x)
$$

It follows from (1.2.4) and (1.3.3) that, for all $\beta \in \Delta$ the root function $f_{\beta}^{Q}$ admits a unique well-defined continuous extension $f_{\beta}^{Q}: D[Q] \rightarrow(0, \infty]$ by defining, for any $y \in D_{P(I)}$

$$
f_{\beta}^{Q}(y)= \begin{cases}\infty & \text { for } \quad \beta \in \Delta-I \\ f_{\beta}^{\bar{Q}}(y) & \text { for } \quad \beta \in I-J \\ 1 & \text { for } \beta \in J .\end{cases}
$$

Similarly, the projection $\Phi_{Q}: D \rightarrow D_{Q}$ factors,

$$
\Phi_{Q}(x)=\Phi_{\bar{Q}} \circ \Phi_{P}(x)=m \cdot \Phi_{Q}\left(x_{0}\right) \in D_{Q} \cong M_{Q} / K_{Q}
$$

so it also has a unique continuous extension to the neighborhood $D[Q]$ which we denote by the same symbol, $\Phi_{Q}: D[Q] \rightarrow D_{Q}$.

From Lemma 1.2.7, we obtain a characterization for convergence in the reductive Borel-Serre compactification:

LEMMA RBS 1.3.6. Let $\mathbf{Q}_{0}$ be the standard minimal parabolic subgroup of $\mathbf{G}$ and let $\mathbf{P}=\mathbf{P}(\mathbf{I})$ be the standard parabolic subgroup corresponding to a subset $I \subset \Delta$. Let $x_{\infty} \in D_{P}$. Then a sequence of points $\left\{x_{k}\right\} \subset D$ converges (in the reductive Borel-Serre compactification) to $x_{\infty}$ iff the following two conditions hold:

(1) $\Phi_{P}\left(x_{k}\right) \in D_{P}$ converges in $D_{P}$ to $x_{\infty}$.

(2) $f_{\beta}^{Q_{0}}\left(x_{k}\right) \rightarrow \infty$ for all $\beta \in \Delta-I$.

Moreover, in the presence of (1), condition (2) is equivalent to the condition

$\left(2^{\prime}\right) \quad f_{\beta}^{P}\left(x_{k}\right) \rightarrow \infty$ for all $\beta \in \Delta-I$.

Proof 1.3.7 This is because, within any set $\Phi_{P}^{-1}$ (compact), the ratio $f_{\beta}^{Q_{0}}: f_{\beta}^{P}$ is bounded. To be precise, use (1.1.12) to write $x_{k}=u_{k} w_{k} a_{k} b_{k} m_{k} \cdot x_{0}$ with $u_{k} \in \mathcal{U}_{P}$, $w_{k} \in i_{x_{0}}\left(\mathcal{U}_{\bar{Q}_{0}}\right), a_{k} \in A_{P}\left(x_{0}\right), b_{k} \in A_{Q_{0}}\left(x_{0}\right) \cap M_{P}\left(x_{0}\right)$, and $m_{k} \in M_{Q_{0}}\left(x_{0}\right)$. Then $\Phi_{P}\left(x_{k}\right)=w_{k} b_{k} m_{k} \cdot \Phi_{P}\left(x_{0}\right)$ which converges; hence the $b_{k}$ converge. So by (1.3.2) we conclude that $f_{\beta}^{P}\left(x_{k}\right)=\beta\left(a_{k}\right) \rightarrow \infty$ iff $f_{\beta}^{Q_{0}}\left(x_{k}\right)=\beta\left(a_{k}\right) \beta\left(b_{k}\right) \rightarrow \infty$. 
We shall also need the following consequence of (1.3.5) and Lemma RBS 1.3.6,

CONSEQUENCE 1.3.8. Let $\mathbf{P}=\mathbf{P}(\mathbf{I})$ be the standard parabolic subgroup corresponding to a subset $I \subset \Delta$, and let $x_{\infty} \in \bar{D}_{P}^{R B S} \subset \bar{D}^{R B S}$. Suppose $\left\{x_{k}\right\} \subset D$ is a sequence which converges in $\bar{D}^{R B S}$ to $x_{\infty}$. Then the sequence $\left\{\Phi_{P}\left(x_{k}\right)\right\} \subset D_{P}$ also converges to $x_{\infty}$ in $\bar{D}^{R B S}$.

1.3.9. The action of $\mathbf{G}(\mathbb{Q})$ extends continuously to the partial compactification $\bar{D}^{R B S}$. For any arithmetic group $\Gamma \subset \mathbf{G}(\mathbb{Q})$ the quotient $\bar{X}^{R B S}=\Gamma \backslash \bar{D}^{R B S}$ is called the reductive Borel-Serre compactification of $X=\Gamma \backslash D$. The image $X_{P}$ of the boundary component $D_{P}$ is called a boundary stratum, of which there are finitely many: one for each $\Gamma$-conjugacy class of rational parabolic subgroups $\mathbf{P} \subset \mathbf{G}$. If $\Gamma$ is neat then $\bar{X}^{R B S}$ is (Whitney-)stratified by these boundary strata. The projection $\bar{D}^{B S} \rightarrow \bar{D}^{R B S}$ passes to a surjective stratum preserving mapping $\bar{X}^{B S} \rightarrow \bar{X}^{R B S}$ whose restriction to $X$ is the identity.

2. Linear symmetric spaces. The main result in this section is Theorem $B$ (§2.8) which describes the topology of the reductive Borel-Serre compactification of certain convex polyhedral cones.

2.1. Throughout this section, $\mathbf{G}$ denotes a connected reductive algebraic group defined over $\mathbb{Q}$, and $\rho: \mathbf{G} \rightarrow G L(\mathbf{V})$ denotes a faithful rational representation of $\mathbf{G}$ on some rational vectorspace $\mathbf{V}$. Let $G=\mathbf{G}(\mathbb{R})^{0}$ denote the connected component of the group of real points. We assume that $G$ acts with an open orbit $C \subset V=\mathbf{V} \otimes_{\mathbb{Q}} \mathbb{R}$ such that the stabilizer $K=\operatorname{Stab}_{G}(e)$ of a chosen basepoint $e \in C$ is a maximal compact subgroup of $G$. Then we may identify the group $G \cong \operatorname{Aut}^{0}(C) \subset G L(V)$ with the connected component of the group of linear automorphisms of $V$ which preserve the orbit $C$. The vectorspace $V$ admits a rational inner product $\langle\cdot, \cdot\rangle$ so that

$$
\check{C}=\{x \in V \mid\langle x, c\rangle>0 \forall c \in \bar{C}-\{0\}\}
$$

coincides with $C$ (and $G=G^{t} \subset G L(V)$ ). Then $C$ is a self adjoint homogeneous rational cone in $V$. We shall assume for simplicity that $C$ is irreducible over $\mathbb{Q}$ which implies that the split component $A_{G}=\mathbf{S}_{\mathbf{G}}(\mathbb{R})^{0}$ is 1-dimensional, and acts on $V$ by homotheties. (The results of this chapter easily extend to the case that $C$ is reducible, and will eventually be applied to the group $\mathbf{G}_{\ell}$ of Section 4.)

Fix once and for all a basepoint $e \in C$ which is rational, $e \in \mathbf{V}(\mathbb{Q})$. Let $\mathfrak{g}=\mathfrak{k} \oplus \mathfrak{p}$ denote the Cartan decomposition corresponding to the choice of maximal compact subgroup $K=\operatorname{Stab}_{G}(e)$ and let $\phi: \mathfrak{g} \rightarrow \operatorname{End}(V)$ denote the differential of $\rho$. Then $\phi$ determines an isomorphism $\mathfrak{p} \rightarrow V$ by $x \mapsto \phi(x)(e)$ whose inverse 
we denote by $a \mapsto T_{a} \in \mathfrak{p}$. The vectorspace $V$ admits a Jordan algebra structure such that, for any $x \in \mathfrak{p}$, the mapping $\phi(x) \in \operatorname{End}(V)$ is Jordan multiplication by the element $\phi(x)(e) \in V$. In other words, $a \bullet b=\phi\left(T_{a}\right) b$ for all $a, b \in V$. It is customary to drop the explicit mention of $\phi$ and to write $a \bullet b=T_{a}(b)$. The basepoint $e \in C \subset V$ is the identity element of the Jordan algebra. For all $a, b, x \in V$ and for all $s \in \mathbb{R}$ we have $T_{a+s b}(x)=T_{a}(x)+s T_{b}(x)$. The cone $C$ is given by $C=\left\{x^{2} \mid x \in V\right.$ is invertible $\}$ and its closure is $\bar{C}=\left\{x^{2} \mid x \in V\right\}$.

2.2. Standard partial compactification. The standard partial compactification of $C$ is the Satake partial compactification which corresponds to the representation $\rho$. It may be explicitly described as follows.

For each idempotent $\epsilon \in V$ the associated endomorphism (given by Jordan multiplication) $T_{\epsilon}: V \rightarrow V$ is semisimple with eigenvalues $0, \frac{1}{2}$, and 1 . The "Peirce decomposition" $V \cong V_{0} \oplus V_{\frac{1}{2}} \oplus V_{1}$ is the corresponding eigenspace decomposition. Define boundary components $C_{0}(\epsilon)=\operatorname{int}\left(\bar{C} \cap V_{0}\right)$ and $C_{1}(\epsilon)=$ $C_{0}(e-\epsilon)=\operatorname{int}\left(\bar{C} \cap V_{1}\right)$. These boundary components are rational if the corresponding idempotents $\epsilon$ and $e-\epsilon$ are rational elements of $V$. The subgroup $P=\operatorname{Norm}_{G}\left(C_{1}(\epsilon)\right)$ which preserves $C_{1}(\epsilon)$ is a maximal parabolic subgroup of $G$. If $\epsilon$ is rational, then $P$ is the real points of a rationally defined maximal parabolic subgroup $\mathbf{P}$ of $\mathbf{G}$. Conversely, every maximal rational parabolic subgroup $P \subset G$ preserves a unique rational boundary component.

The closure $\bar{C} \subset V$ is the disjoint union of $C$ and all its boundary components. Let $C^{*} \subset V$ denote the union of $C$ and all its rational boundary components. Define the Satake topology on $C^{*}$ to be the unique topology so that: (1) for any Siegel set $\Omega \subset C$ its closure $\bar{\Omega} \subset V$ in $V$ coincides with its closure $\bar{\Omega}^{\text {Sat }}$ in the Satake topology, and (2) if $y \in C^{*}$ is a point on the boundary, then for any arithmetic group $\Gamma \subset \mathbf{G}(\mathbb{Q})$, there exists a basis of neighborhoods $U$ of $y$ (in the Satake topology) such that $\Gamma_{y} U=U$ where $\Gamma_{y}$ denotes the stabilizer of $y$ in $\Gamma$. The existence of such a topology is essentially proven in [S1] $\S 2$, [BB] Thm. 4.9, or [Z2] p. 340, although their proofs must be modified slightly so as to apply to our $\mathbf{G}$ which is reductive, rather than semisimple. The standard partial compactification $\bar{C}^{\text {std }}$ of $C$ is the space $C^{*}$ with the Satake topology. For any rational idempotent $\epsilon \in V$ the closure of $C_{1}(\epsilon)$ in $\bar{C}^{s t d}$ is the standard partial compactification of $C_{1}(\epsilon)$.

Throughout $\S 2$ and $\S 3$ we let $D=C / A_{G}$ denote the quotient of $C$ under homotheties. Denote also by $\bar{D}^{s t d}=\bar{C}^{s t d} / A_{G}$ (with the Satake topology) the quotient under homotheties. For any subset $S \subset C$ we denote by $[S] \subset D$ its image in $D$. The group $\mathbf{G}(\mathbb{Q})$ of rational points acts on the partial compactification $\bar{D}^{\text {std }}$.

Proposition 2.2.1. For any arithmetic subgroup $\Gamma \subset G$ the quotient $\Gamma \backslash \bar{D}^{\text {std }}$ is compact. If $\Gamma$ is neat, then $\Gamma \backslash \bar{D}^{\text {std }}$ is a stratified space with one stratum $\Gamma \cap$ $P(\epsilon) \backslash C_{1}(\epsilon) / A_{G}$ for each $\Gamma$ conjugacy class of rational boundary components $C_{1}(\epsilon)$. 
2.3. Roots. Throughout the rest of this section we fix a "standard" minimal rational parabolic subgroup $\mathbf{Q}_{0} \subset \mathbf{G}$. This corresponds to a choice of a complete set of mutually orthogonal rational idempotents $\left\{\epsilon_{1}, \epsilon_{2}, \ldots, \epsilon_{r+1}\right\}$ (in other words, $\epsilon_{i} \bullet \epsilon_{j}=0$ for $i \neq j, \epsilon_{i}^{2}=\epsilon_{i}$, and $\left.\epsilon_{1}+\epsilon_{2}+\cdots+\epsilon_{r+1}=e\right)$. The $\epsilon_{i}$ may be ordered so that $Q_{0}$ is the normalizer of the "complete" flag of rational boundary components

$$
\bar{C}_{1}\left(\epsilon_{1}\right) \subset \bar{C}_{1}\left(\epsilon_{1}+\epsilon_{2}\right) \subset \cdots \subset \bar{C}_{1}\left(\epsilon_{1}+\cdots+\epsilon_{r+1}\right)=\bar{C} .
$$

The canonical lift of $A_{Q_{0}}$ (determined by the basepoint $e \in C$ ) is $A_{Q_{0}}(e)=$ $\exp \left(\sum \mathbb{R} T_{\epsilon_{i}}\right)$. If $\gamma_{i} \in \operatorname{Hom}\left(A_{Q_{0}}, \mathbb{R}\right)$ denotes the dual basis to the elements $\exp \left(T_{\epsilon_{i}}\right)$ then the rational roots $\Phi\left(A_{Q_{0}}, G\right)$ of $G$ are $\left\{\frac{1}{2}\left(\gamma_{i}-\gamma_{j}\right)\right\}$ for $i \neq j$ and the simple roots $\Delta=\Delta_{Q_{0}}$ (appearing in the unipotent radical of $Q_{0}$ ) are

$$
\alpha_{i}=\frac{1}{2}\left(\gamma_{i}-\gamma_{i+1}\right) \text { for } 1 \leq i \leq r
$$

The Dynkin diagram for $G$ is linear and it corresponds to the ordering $\alpha_{1}, \alpha_{2}, \ldots$, $\alpha_{r}$ of the simple roots (or to the ordering $\epsilon_{1}, \epsilon_{2}, \ldots, \epsilon_{r}, \epsilon_{r+1}$ of the idempotents).

2.4. Reductive Borel-Serre boundary components. The immediate goal is Lemma 2.4.11 which describes the closure of a single boundary component in the reductive Borel-Serre compactification. Throughout $\$ 2.4$ we fix a standard parabolic subgroup $\mathbf{Q} \supset \mathbf{Q}_{0}$ corresponding to a subset $I \subset \Delta$ of the simple roots as in (1.1.6). Write $\Delta-I=\left\{\alpha_{m_{1}}, \alpha_{m_{2}}, \ldots, \alpha_{m_{q}}\right\}$ (where $1 \leq m_{1}<m_{2}<\cdots<$ $\left.m_{q} \leq r\right)$. Then we obtain orthogonal idempotents

$$
\begin{aligned}
d_{1} & =\epsilon_{1}+\epsilon_{2}+\cdots+\epsilon_{m_{1}} \\
d_{2} & =\epsilon_{m_{1}+1}+\epsilon_{m_{1}+2}+\cdots+\epsilon_{m_{2}} \\
\cdots & \\
d_{q+1} & =\epsilon_{m_{q}+1}+\epsilon_{m_{q}+2}+\cdots+\epsilon_{r+1}
\end{aligned}
$$

so that $Q$ is the normalizer of the (partial rational) flag of boundary components

$$
\bar{C}_{1}\left(d_{1}\right) \subset \bar{C}_{1}\left(d_{1}+d_{2}\right) \subset \cdots \subset \bar{C}_{1}\left(d_{1}+\cdots+d_{q+1}\right)=\bar{C} .
$$

The canonical lift $A_{Q}(e)$ of the torus $A_{Q}$ may be parametrized by elements

$$
\lambda_{Q}\left(t_{1}, t_{2}, \ldots, t_{q+1}\right)=\exp \left(s_{1} T_{d_{1}}+s_{2} T_{d_{2}}+\cdots+s_{q+1} T_{d_{q+1}}\right)
$$

where $t_{i}=e^{\frac{1}{2} s_{i}}$. The torus $A_{G}=\mathbf{Z}_{\mathbf{G}}(\mathbb{R})^{0}$ is given by $\lambda_{Q}(t, t, \ldots, t)$. It follows from(2.3.2) that, when viewed as characters on $A_{Q}$, the restrictions of the simple 
roots $\Delta-I=\left\{\alpha_{m_{1}}, \alpha_{m_{2}}, \ldots, \alpha_{m_{q}}\right\}$ to $A_{Q}$ are given by

$$
\alpha_{m_{j}}\left(\lambda_{Q}\left(t_{1}, t_{2}, \ldots, t_{q+1}\right)\right)=t_{j} t_{j+1}^{-1} \text { for } 1 \leq j \leq q
$$

Let

$$
V=\bigoplus_{1 \leq i \leq j \leq q+1} V_{i j}
$$

denote the (simultaneous) Peirce decomposition of $V$ relative to this collection $d_{1}+d_{2}+\cdots+d_{q+1}=e$ of idempotents, where $V_{i i}=V_{1}\left(d_{i}\right)$ and $V_{i j}=V_{\frac{1}{2}}\left(d_{i}\right) \cap$ $V_{\frac{1}{2}}\left(d_{j}\right)$ for $i \neq j$ (cf. [AMRT] II, 3.8, p. 92 or [FK] Thm. IV.2.1, p. 68). Then $\lambda_{Q}^{2}\left(t_{1}, t_{2}, \ldots, t_{q+1}\right)$ acts on $V_{i j}$ with eigenvalue $t_{i} t_{j}$ for $1 \leq i, j \leq q+1$. The Jordan algebra structure on $V$ restricts to a rationally defined Jordan algebra structure on each $V_{i i}=V_{1}\left(d_{i}\right) \subset V$ with identity element $d_{i}$ and with self adjoint homogeneous cone $C_{1}\left(d_{i}\right)=\bar{C} \cap V_{1}\left(d_{i}\right)$ (for $\left.1 \leq i \leq q+1\right)$. Let $G_{i}=\operatorname{Aut}^{0}\left(C_{1}\left(d_{i}\right), V_{i i}\right)$. Then $G_{i}$ are the real points of a rationally defined algebraic group $\mathbf{G}_{i}$ and $C_{1}\left(d_{i}\right) \cong G_{i} / K_{i}$ where $K_{i}$ is the isotropy subgroup in $G_{i}$ of the basepoint $d_{i}$. Let $p_{i}: V \rightarrow V_{i i}$ denote the linear projection which is determined by the Peirce decomposition. Define

$$
\psi_{i}: L_{Q}(e) \rightarrow G_{i}
$$

by $\psi_{i}(g)=g \mid C_{1}\left(d_{i}\right)$. Thus there are two projections $C \rightarrow C_{1}\left(d_{i}\right)$ : one given by the linear projection $p_{i}$ and the second, $\phi_{i}$, given by the composition $C=$ $Q / K_{Q} \rightarrow L_{Q} / K_{Q} \rightarrow G_{i} / K_{i}$. The following lemma says that these projections agree on points $x=g \cdot e$ which are in the orbit of the Levi subgroup $L_{Q}(e)$; the discrepancy between these two projections is analyzed in the proof of Proposition 2.6.2.

LEMMA 2.4.7. For all $g \in L_{Q}(e)$ and for all $i(1 \leq i \leq q+1)$ we have

$$
p_{i}(g \cdot e)=\phi_{i}(g \cdot e)=\psi_{i}(g) \cdot d_{i} \in C_{1}\left(d_{i}\right)
$$

Proof 2.4.8. Since $L_{Q}(e)$ is the centralizer of $A_{Q}(e)$ it follows that each $V_{i i}$ is preserved by $L_{Q}(e)$ and that the projection $p_{i}$ commutes with the action of $g \in L_{Q}(e)$ on $V_{i i}$. Furthermore, $p_{i}(e)=d_{i}$.

The mapping

$$
\psi_{Q}: L_{Q}(e) \rightarrow G_{1} \times G_{2} \times \cdots \times G_{q+1}
$$


given by

$$
\psi_{Q}(g)=\left(g\left|C_{1}\left(d_{1}\right), g\right| C_{1}\left(d_{2}\right), \ldots, g \mid C_{1}\left(d_{q+1}\right)\right)
$$

is surjective with compact kernel ([AMRT] II, 3.9 Prop. 10) and it induces diffeomorphisms $C_{Q}=L_{Q} / K_{Q} \cong C_{1}\left(d_{1}\right) \times \cdots \times C_{1}\left(d_{q+1}\right)$ and

$$
\Psi_{Q}: D_{Q} \cong D_{1} \times \cdots \times D_{q+1}
$$

where $D_{i}=C_{1}\left(d_{i}\right) /$ homotheties for $1 \leq i \leq q+1$.

LEMMA 2.4.11. The mapping $\psi_{Q}$ induces a homeomorphism (which is smooth on each boundary component) between the reductive Borel-Serre partial compactifications,

$$
\bar{\Psi}_{Q}: \bar{D}_{Q}^{R B S} \cong \bar{D}_{1}^{R B S} \times \bar{D}_{2}^{R B S} \times \cdots \times \bar{D}_{q+1}^{R B S}
$$

Proof 2.4.13. Each parabolic subgroup of $G_{1} \times G_{2} \times \cdots \times G_{q+1}$ is of the form $R_{1} \times R_{2} \times \cdots \times R_{q+1}$ with $R_{i}$ parabolic in $G_{i}$.

2.4.14. The next immediate goal is Corollary 2.6.3 which describes the projection from the reductive Borel-Serre compactification to the standard compactification. To describe this projection we must associate to each rational parabolic subgroup of $\mathbf{G}$ a maximal rational parabolic subgroup.

The RBS boundary component $D_{Q}$ appears in $\bar{D}_{P}^{R B S}$ for every parabolic subgroup $P \supset Q$. However the ordering of the roots determines an ordering of the maximal parabolic subgroups containing $Q$. Define

$$
P=Q^{\dagger}=\operatorname{Norm}\left(C_{1}\left(d_{1}\right)\right)
$$

to be the first maximal parabolic subgroup in this ordering. It corresponds to the single idempotent $d_{1}$. Set $C_{0}=C_{1}\left(e-d_{1}\right), G_{0}=\operatorname{Aut}\left(C_{0}, V_{0}\left(d_{1}\right)\right), D_{0}=$ $C_{0} /$ homotheties (and $C_{1}=C_{1}\left(d_{1}\right), G_{1}=\operatorname{Aut}\left(C_{1}, V_{1}\left(d_{1}\right)\right), D_{1}=C_{1} /$ homotheties). As in (2.4.9) and Lemma 2.4.11 set $\psi_{P}: L_{P} \rightarrow G_{1} \times G_{0}$ and $\bar{\Psi}_{P}: \bar{D}_{P}^{R B S \cong}$ $\bar{D}_{1}^{R B S} \times \bar{D}_{0}^{R B S}$.

Lemma 2.4.16. Suppose $Q^{\dagger}=P=\mathcal{U}_{P} G_{1} G_{0}$ as above. There exists a rational parabolic subgroup $H \subset G_{0}$ with corresponding reductive Borel-Serre boundary component $D_{0, H} \subset \bar{D}_{0}^{R B S}$ so that

$$
\bar{\Psi}_{P}\left(D_{Q}\right)=D_{1} \times D_{0, H} \subset D_{1} \times \bar{D}_{0}^{R B S} .
$$


Proof 2.4.17. The image of $Q$ under the composition

$$
Q \subset P \underset{\nu_{P}}{\longrightarrow} L_{P} \underset{\psi_{P}}{\longrightarrow} G_{1} \times G_{0}
$$

is of the form $G_{1} \times H$ for some parabolic subgroup $H \subset G_{0}$.

2.4.18. These spaces and mappings fit together in the following diagram. The composition across the top row is $\phi_{i}$ and the composition across the middle row is $\Phi_{i}$.

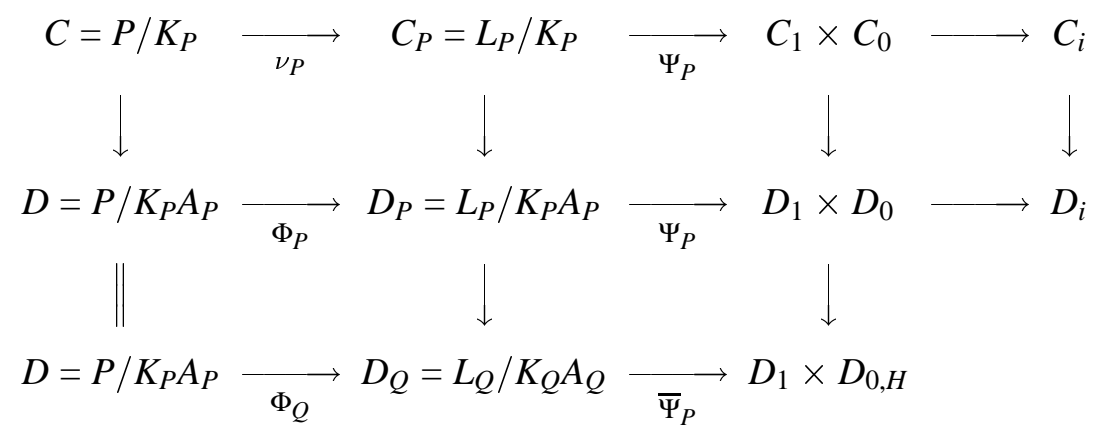

2.5. Peirce coordinates. The Peirce decomposition gives rise to a coordinate system on $C$ which is analogous to the Siegel coordinate system of PiatetskiShapiro for the case of Hermitian symmetric spaces. Let $\mathbf{P} \supset \mathbf{Q}_{0}$ be a standard maximal rational parabolic subgroup of $\mathbf{G}$, (see (2.3.2)) say, $\mathbf{P}=\mathbf{P}\left(\Delta-\left\{\alpha_{k}\right\}\right.$ ) for some simple root $\alpha_{k} \in \Delta=\Delta_{Q_{0}}$. Then $P=\operatorname{Norm}_{G}\left(C_{1}(\epsilon)\right)$ where $\epsilon=\epsilon_{1}+\epsilon_{2}+\cdots+\epsilon_{k}$. Let $V=V_{1}(\epsilon) \oplus V_{\frac{1}{2}}(\epsilon) \oplus V_{0}(\epsilon)$ denote the resulting Peirce decomposition of $V$ (with into eigenspaces of $\left.T_{\epsilon}\right)$. Let $\lambda_{P}\left(t_{1}, t_{0}\right)=\exp \left(s_{1} T_{\epsilon}+s_{0} T_{e-\epsilon}\right)$ denote the parametrization of $A_{P}$ as in (2.4.3), with $t_{i}=e^{\frac{1}{2} s_{i}}$ for $i=1,0$. Let $\mathrm{V}=\left(\mathrm{V}_{1}, \mathrm{v}_{\frac{1}{2}}, \mathrm{v}_{0}\right) \in$ $V_{1} \oplus V_{\frac{1}{2}} \oplus V_{0}$.

LEMMA 2.5.1. In these coordinates, the action of $P$ on $V$ is given by

$$
g \cdot \mathrm{v}=\left(\begin{array}{ccc}
A & M & N \\
0 & C & D \\
0 & 0 & B
\end{array}\right)\left(\begin{array}{c}
\mathrm{v}_{1} \\
\mathrm{v}_{\frac{1}{2}} \\
\mathrm{v}_{0}
\end{array}\right)
$$

(where $A, M, N, C, D$, and $B$ are linear mappings which depend on $g$.) Furthermore,

(1) $g \in L_{P}$ iff $M=0, N=0, D=0$.

(2) The element $g=\lambda_{P}\left(t_{1}, t_{0}\right) \in A_{P}$ is given by

$$
\lambda_{P}\left(t_{1}, t_{0}\right)=\left(\begin{array}{ccc}
t_{1}^{2} & 0 & 0 \\
0 & t_{1} t_{0} & 0 \\
0 & 0 & t_{0}^{2}
\end{array}\right) .
$$


(3) The root function $f_{\alpha_{k}}^{P}$ is given by

$$
f_{\alpha_{k}}^{P}\left(u \lambda_{P}\left(t_{1}, t_{0}\right) m e\right)=t_{1} t_{0}^{-1}
$$

for any $u \in \mathcal{U}_{P}, \lambda_{P}\left(t_{1}, t_{0}\right) \in A_{P}$ and $m \in M_{P}$.

(4) If $g \in \mathcal{U}_{P}$ then $A=I$ and $B=I$.

(5) The orbit of the basepoint $e=(\epsilon, 0, e-\epsilon)$ under $L_{P}$ is the product $C_{1}(\epsilon) \times$ $\{0\} \times C_{1}(e-\epsilon)$.

Moreover, there exists a family of bilinear forms $h_{t}: V_{\frac{1}{2}}(\epsilon) \times V_{\frac{1}{2}}(\epsilon) \rightarrow V_{0}(\epsilon)$ depending smoothly on a parameter $t \in C_{1}(\epsilon)$ so that the cone $C$ is given by

$$
C=\left\{(t, w, z) \in V_{1} \oplus V_{\frac{1}{2}} \oplus V_{0} \mid t \in C_{1} \text { and } z-h_{t}(w, w) \in C_{0}(\epsilon)\right\}
$$

in analogy with the well-known inequality (cf. (7.5.2)) which, in the case of a Hermitian symmetric space, defines a Siegel domain.

Proof 2.5.2. The 1-parameter group $\lambda(t)=\lambda\left(t_{1}, 1\right)=\exp \left(s_{1} T_{\epsilon}\right)$ is in the center of the Levi quotient $L_{P}$ and it has the property that (cf. [AMRT] II, 3.3 and $[\mathrm{MF}]$ Ch. $2 \S 2$ ),

$$
P=\left\{g \in G \mid \lim _{t \rightarrow 0} \lambda(t) g \lambda(t)^{-1} \text { exists }\right\}
$$

Then $\lambda(t) \cdot\left(\mathrm{v}_{1}, \mathrm{v}_{\frac{1}{2}}, \mathrm{v}_{0}\right)=\left(t^{2} \mathrm{v}_{1}, t^{1} \mathrm{v}_{\frac{1}{2}}, \mathrm{v}_{0}\right)$. Writing

$$
g=\left(\begin{array}{lll}
A & M & N \\
U & C & D \\
V & W & B
\end{array}\right)
$$

we see that

$$
\lambda(t) g \lambda(t)^{-1}=\left(\begin{array}{ccc}
A & t M & t^{2} N \\
t^{-1} U & C & t D \\
t^{-2} V & t^{-1} W & B
\end{array}\right)
$$

which implies that $U=0, V=0$, and $W=0$. Moreover, $g \in L_{P}(e)=Z_{P}(\lambda(t))$ if and only if $\lambda(t) g \lambda(t)^{-1}=g$ for all $t$, which implies that $M=0, N=0$, and $D=0$. This proves parts (1) and (5). Part (2) follows from the definition of $\lambda\left(t_{1}, t_{0}\right)$ and 
part (3) is a restatement of (2.4.4). Part (4) follows from the observation that the projection

$$
\left(\begin{array}{ccc}
A & M & N \\
0 & C & D \\
0 & 0 & B
\end{array}\right) \mapsto(A, B)
$$

is a group homomorphism $P \rightarrow G_{1} \times G_{0}$ whose restriction to $L_{P}$ agrees with $\psi$ (cf. Lemma 2.4.7). Since $G_{1} \times G_{0}$ is reductive, the unipotent radical $\mathcal{U}_{P}$ is in the kernel of this mapping.

The "moveover" part of this theorem will not be needed in this paper and is included for completeness. It is proven in [T]. The bilinear form $h_{t}(\mathrm{v}, \mathrm{v})$ is in fact given by the "quadratic representation" $[\mathrm{FK}] h_{t}(\mathrm{~V}, \mathrm{v})=P(\mathrm{v})(t)=2 \mathrm{~V}(\mathrm{~V} t)-\mathrm{v}^{2} t$.

2.6. Convergence. Fix any point $w \in \bar{D}^{R B S}$. Then $w$ lies in some RBS boundary component, say $D_{Q}$, with normalizing parabolic group $Q=\mathbf{Q}(\mathbb{R})$. Let $\mathbf{P}=\mathbf{Q}^{\dagger}$ (cf. (2.4.15)) be the maximal parabolic subgroup which is first among the ordering of all maximal parabolic subgroups containing $\mathbf{Q}$. Then $P=\operatorname{Norm}\left(C_{1}(\epsilon)\right)$ for some rational idempotent $\epsilon$ (denoted $d_{1}$ in $\$ 2.4$.14) which determines a Peirce decomposition $V=V_{1}(\epsilon) \oplus V_{\frac{1}{2}}(\epsilon) \oplus V_{0}(\epsilon)$. If $\mathrm{v}=\left(\mathrm{v}_{1}, \mathrm{v}_{\frac{1}{2}}, \mathrm{v}_{0}\right) \in V$ then we will write $[\mathrm{v}]=\left[\mathrm{V}_{1}: \mathrm{V}_{\frac{1}{2}}: \mathrm{v}_{0}\right] \in D$ for its homothety class. Set $C_{i}=\bar{C} \cap V_{i}(\epsilon)$, $D_{i}=C_{i} /$ homotheties, and $G_{i}=\operatorname{Aut}^{0}\left(C_{i}, V_{i}(\epsilon)\right)$ for $i=1,0$. Then (2.4.9) $\psi_{P}: L_{P} \rightarrow$ $G_{1} \times G_{0}$ induces $\bar{\Psi}_{P}: \bar{D}_{P}^{R B S} \cong \bar{D}_{1}^{R B S} \times \bar{D}_{2}^{R B S}$. The linear projections $p_{i}: V \rightarrow V_{i}(\epsilon)$ determine projections $p_{i}: C \rightarrow C_{i}$ and $p_{i}: D \rightarrow D_{i}$ (for $i=1,0$ ). By Lemma 2.4.16 we have

$$
\bar{\Psi}_{P}\left(D_{Q}\right)=D_{1} \times D_{0, H} \subset D_{1} \times \bar{D}_{0}^{R B S}
$$

for some rational parabolic subgroup $H \subset G_{0}$, and we write $\bar{\Psi}_{P}(w)=\left(w_{1}, w_{0}\right)$.

Proposition 2.6.2. Let $\left\{y_{k}\right\} \subset D$ be a sequence. If $y_{k}$ converges to $w$ in the reductive Borel Serre compactification $\bar{D}^{R B S}$ then the following two conditions hold,

(1) The sequence $y_{k}$ converges to $w_{1} \in D_{1} \subset \bar{D}^{\text {std }}$ in the standard compactification of $D$ and

(2) the sequence $p_{0}\left(y_{k}\right)$ converges to $w_{0} \in D_{0, H} \subset \bar{D}_{0}^{R B S}$ in the reductive Borel-Serre compactification of $D_{0}$.

Conversely, suppose $\left\{y_{k}\right\} \subset D$ is contained in a Siegel set and satisfies conditions (1) and (2) above. Then $y_{k} \rightarrow w$ in $\bar{D}^{R B S}$.

COROLlary 2.6.3 The identity mapping $D \rightarrow D$ has a unique continuous extension

$$
\mu=\mu_{G}: \bar{D}^{R B S} \rightarrow \bar{D}^{s t d}
$$


which takes boundary components to boundary components. For any maximal rational parabolic subgroup $P=P(\epsilon) \subset G$ the restriction $\mu \mid \bar{D}_{P}^{R B S}$ is given by the composition

$$
\bar{D}_{P}^{R B S} \underset{\bar{\Psi}_{P}}{\longrightarrow} \bar{D}_{1}^{R B S} \times \bar{D}_{0}^{R B S} \underset{\pi_{1}}{\longrightarrow} \bar{D}_{1}^{R B S} \underset{\mu_{G_{1}}}{\longrightarrow} \bar{D}_{1}^{s t d}
$$

(where $D_{i}=C_{i}(\epsilon) /$ homotheties and with $\bar{\Psi}_{P}$ as in (§2.4.18)). A RBS boundary component $D_{Q}$ is contained in $\mu^{-1}\left(D_{1}\right)$ iff $Q^{\dagger}=P$.

Corollary 2.6.3 is essentially proven in [Z2], however we need a slightly more explicit version than appears in [Z2].

2.6.4. Proof of Proposition 2.6.2. For $i=1,0$, denote by $\Phi_{i}: D \rightarrow D_{i}$ the composition along the middle row in the diagram in $\S 2.4 .18$. The proposition is not trivial because the mapping $\Phi_{1}: C \rightarrow C_{1}$ does not necessarily agree with the linear projection $p_{1}:\left(\mathrm{v}_{1}, \mathrm{v}_{\frac{1}{2}}, \mathrm{v}_{0}\right) \mapsto \mathrm{v}_{1}$ given by the Peirce decomposition. For $\mathrm{v} \in C$ write $\mathrm{v}=$ uame relative to the Langlands decomposition $P=\mathcal{U}_{P} A_{P}(e) M_{P}(e)$ of $P$. Using Lemma 2.5.1, write

(2.6.5) $m=\left(\begin{array}{ccc}A & 0 & 0 \\ 0 & C & 0 \\ 0 & 0 & B\end{array}\right), a=\lambda_{P}\left(t_{1}, t_{0}\right)=\left(\begin{array}{ccc}t_{1}^{2} & 0 & 0 \\ 0 & t_{1} t_{0} & 0 \\ 0 & 0 & t_{0}^{2}\end{array}\right), u=\left(\begin{array}{ccc}I & M^{\prime} & N^{\prime} \\ 0 & C^{\prime} & D^{\prime} \\ 0 & 0 & I\end{array}\right)$

relative to the Peirce decomposition $V=V_{1}(\epsilon) \oplus V_{\frac{1}{2}}(\epsilon) \oplus V_{0}(\epsilon)$. Then

$$
\mathrm{V}=\operatorname{uam}\left(\begin{array}{c}
\epsilon \\
0 \\
e-\epsilon
\end{array}\right)=\left(\begin{array}{c}
t_{1}^{2} A \epsilon+t_{0}^{2} N^{\prime} B(e-\epsilon) \\
t_{0}^{2} D^{\prime} B(e-\epsilon) \\
t_{0}^{2} B(e-\epsilon)
\end{array}\right)
$$

the first and last coordinates of which are $p_{1}(\mathrm{v})$ and $p_{0}(\mathrm{v})$ respectively. On the other hand by Lemma 2.4.7,

$$
\Psi_{P} \nu_{P}(\mathrm{v})=\Psi_{P}(\text { ame })=\left(t_{1}^{2} A \epsilon, t_{0}^{2} B(e-\epsilon)\right) \in C_{1}(\epsilon) \times C_{1}(e-\epsilon) .
$$

Hence,

$$
\left[p_{0}(\mathrm{v})\right]=\Phi_{0}([\mathrm{v}]) \in D_{0},
$$

but $\left[p_{1}(\mathrm{v})\right] \not=\Phi_{1}([\mathrm{v}]) \in D_{1}$ unless $N^{\prime} B=0$.

Suppose the sequence $\left\{y_{k}\right\}$ converges in $\bar{D}^{R B S}$ to $w \in D_{Q}$. Since $w \in D_{Q} \subset$ $\bar{D}_{P}^{R B S}$ we have, (by Corollary 1.3.8 and Lemma 2.4.11),

$$
\Phi_{P}\left(y_{k}\right) \rightarrow w \text { in } \bar{D}_{P}^{R B S} \cong \bar{D}_{1}^{R B S} \times \bar{D}_{0}^{R B S}
$$


It follows that $\Phi_{0}\left(y_{k}\right) \rightarrow w_{0}$ in ${\overline{D_{0}}}^{R B S}$ (and that $\Phi_{1}\left(y_{k}\right) \rightarrow w_{1}$ in $D_{1}^{R B S}$ ). By (2.6.7) this implies that $p_{0}\left(y_{k}\right) \rightarrow w_{0}$ which proves (2).

To prove (1), we may assume the parabolic subgroup $Q$ is standard, that it corresponds to a subset $I \subset \Delta$ of the simple roots $\Delta$ which occur in the unipotent radical of $Q_{0}$ (cf. $\S 2.4$ ), and that it normalizes the partial flag (2.4.2) corresponding to the ordered set of orthogonal idempotents $d_{1}+d_{2}+\cdots+d_{q+1}=e$. Write

$$
y_{k}=u_{k} a_{k} m_{k}[e]
$$

relative to the canonical Langlands decomposition $Q=\mathcal{U}_{Q} A_{Q}(e) M_{Q}(e)$, where $[e] \in D$ denotes the homothety class of the basepoint $e \in C$. By Lemma RBS 1.3.6, the sequence $m_{k}$ may be chosen so as to converge to some limit $m_{\infty} \in$ $M_{Q}(e)$. If $\Gamma \subset \mathbf{G}(\mathbb{Q})$ is an arithmetic group, then the quotient $\mathcal{U}_{Q} /\left(\Gamma \cap \mathcal{U}_{Q}\right)$ is compact. Hence there exists a compact subset $F \subset \mathcal{U}_{Q}$ such that $\mathcal{U}_{Q}=F$. $\left(\Gamma \cap \mathcal{U}_{Q}\right)$. So we may write $u_{k}=\gamma_{k} u_{k}^{\prime}$ where $\gamma_{k} \in \Gamma \cap \mathcal{U}_{Q}$ stabilizes the point $y_{1} \in D_{1}$ and where $u_{k}^{\prime}$ lies in the fixed compact subset $F \subset \mathcal{U}_{Q}$. The sequence $\left\{y_{k}\right\}$ converges in the Satake topology to $w_{1}$ iff the sequence $\gamma_{k}^{-1} y_{k}=u_{k}^{\prime} a_{k} m_{k}[e] \in D$ also converges to $w_{1}$. So, replacing $y_{k}$ by $\gamma_{k}^{-1} y_{k}$ if necessary, we may (and will) assume the elements $u_{k}$ in equation (2.6.8) remain within some fixed compact subset $F \subset \mathcal{U}_{Q}$. Since the $\left\{m_{k}\right\}$ also remain within some compact set, the elements $y_{k}$ are contained in a Siegel set.

Let us write

$$
V=V_{11} \oplus V_{22} \oplus \cdots \oplus V_{q+1, q+1} \oplus \bigoplus_{1 \leq i<j \leq q+1} V_{i j}
$$

Then the $L_{Q}$ orbit of the basepoint $e=\left(d_{1}, d_{2}, \ldots, d_{q+1}, 0\right)$ is

$$
C_{Q}=C_{1}\left(d_{1}\right) \times C_{1}\left(d_{2}\right) \times \cdots \times C_{1}\left(d_{q+1}\right) \times\{0\},
$$

so the point $m_{k} e$ may be expressed as

$$
m_{k} e=\left(z_{1, k}, z_{2, k}, \ldots, z_{q+1, k}, 0\right) \longrightarrow m_{\infty} e=\left(z_{1, \infty}, z_{2, \infty}, \ldots, z_{q+1, \infty}, 0\right)
$$

As in (2.4.3), write $a_{k}=\lambda\left(t_{1, k}, t_{2, k}, \ldots, t_{q+1, k}\right)$. By (2.4.4), and Lemma 1.3.6 we have

$$
\frac{t_{1, k}}{t_{2, k}} \rightarrow \infty, \frac{t_{2, k}}{t_{3, k}} \rightarrow \infty, \ldots, \frac{t_{q, k}}{t_{q+1, k}} \rightarrow \infty
$$

while

$$
a_{k} m_{k} e=\left(t_{1, k}^{2} z_{1, k}, t_{2, k}^{2} z_{2, k}, \ldots, t_{q+1, k}^{2} z_{q+1, k}, 0\right) .
$$


But $\mathcal{U}_{Q}$ preserves the flag $V_{11}=V_{1}\left(d_{1}\right) \subset V_{1}\left(d_{1}+d_{2}\right) \subset \cdots \subset V$ hence

(2.6.11) $u_{k} a_{k} m_{k} e=\left(t_{1, k}^{2} z_{1, k}\right.$, linear combinations of $\left\{t_{i, k}^{2} z_{i, k}\right\}$ for $\left.i>1\right)$

and the coefficients (of the linear combinations) are restricted to lie in some compact set. But (2.6.10) implies that, modulo homotheties,

$$
y_{k} \longrightarrow\left[z_{1, \infty}: 0: 0: \ldots: 0\right]=w_{1} \in D_{1}
$$

in the "usual" topology. Since $\left\{y_{k}\right\}$ is contained in a Siegel set it follows that $y_{k} \rightarrow w_{1}$ in the Satake topology also. This completes the proof of (1).

Now let us prove the converse, i.e., suppose that $\left\{y_{k}\right\} \subset D$ is contained in a Siegel set, $y_{k} \rightarrow w_{1} \in \bar{D}^{\text {std }}$ and $p_{0}\left(y_{k}\right) \rightarrow w_{0} \in \bar{D}_{0}^{R B S}$. By Lemma 1.3.6, to show that $y_{k} \rightarrow w \in \bar{D}^{R B S}$ we must verify that

(a) $\Phi_{Q}\left(y_{k}\right) \rightarrow w$ and

(b) $f_{\alpha}^{Q}\left(y_{k}\right) \rightarrow \infty$ for all $\alpha \in \Delta-I$.

In order to verify (a) it suffices (see the diagram in $§ 2.4 .18$ ) to show that (i) $\Phi_{1}\left(y_{k}\right) \rightarrow w_{1}$ in $D_{1}$ and (ii) $\Phi_{0}\left(y_{k}\right) \rightarrow w_{0}$ in $\bar{D}_{0}^{R B S}$. But (ii) follows from (2.6.7) and the assumption that $p_{0}\left(y_{k}\right) \rightarrow w_{0}$. So we must verify (i). Choose any lift $\mathrm{v}_{k} \in C$ of $y_{k} \in D$ and write $\mathrm{v}_{k}=u_{k} a_{k} m_{k} e$ relative to the Langlands decomposition $P=\mathcal{U}_{P} A_{P}(e) M_{P}(e)$ of $P$. Since $\left\{\mathrm{v}_{k}\right\}$ lie in a Siegel set, the elements $u_{k}$ and $m_{k}$ lie in some compact set. As in (2.6.5) we may write

$$
a_{k}=\lambda_{P}\left(t_{1, k}, t_{0, k}\right)=\left(\begin{array}{ccc}
t_{1, k}^{2} & 0 & 0 \\
0 & t_{1, k} t_{0, k} & \\
0 & 0 & t_{0, k}^{2}
\end{array}\right)
$$

and

$$
m_{k}=\left(\begin{array}{ccc}
A_{k} & 0 & 0 \\
0 & C_{k} & 0 \\
0 & 0 & B_{k}
\end{array}\right) \text {, and } u_{k}=\left(\begin{array}{ccc}
I & M_{k}^{\prime} & N_{k}^{\prime} \\
0 & C_{k}^{\prime} & D_{k}^{\prime} \\
0 & 0 & I
\end{array}\right)
$$

Each family of matrices $C_{k}, C_{k}^{\prime}, D_{k}^{\prime}, M_{k}^{\prime}, N_{k}^{\prime}$ is contained in some compact set of matrices, while the $A_{k}$ and $B_{k}$ are contained in compact sets of invertible matrices. Since $y_{k} \rightarrow\left[w_{1}: 0: 0\right]$ in the Satake topology, it does so also in the usual topology, so it follows from (2.6.6) that

$$
t_{1, k} t_{0, k}^{-1} \rightarrow \infty
$$

(because the first coordinate dominates the second and third coordinates). From 
this it also follows that $p_{1}\left(y_{k}\right) \rightarrow w_{1}$ in $D_{1}$ and also that

$$
p_{1}\left(u_{k}^{-1} y_{k}\right)=p_{1}\left(a_{k} m_{k}[e]\right)=\left[t_{1, k}^{2} A_{k} \epsilon: 0: t_{0, k}^{2} B_{k}(e-\epsilon)\right] \longrightarrow\left[w_{1}: 0: 0\right]
$$

as well. So, by Lemma 2.4.7 we have

$$
\Phi_{1}\left(y_{k}\right)=\Phi_{1}\left(u_{k}^{-1} y_{k}\right)=p_{1}\left(u_{k}^{-1} y_{k}\right) \longrightarrow w_{1}
$$

which completes the proof of (i) and hence also the proof of (a).

Now let us prove part (b). Write $\Delta-I=\left\{\alpha_{m_{1}}, \alpha_{m_{2}}, \ldots, \alpha_{m_{q+1}}\right\}$ as in $\S 2.4$, and parametrize $A_{Q}(e)$ by elements $\lambda_{Q}\left(t_{1}, t_{2}, \ldots, t_{q+1}\right)$ as in (2.4.3). Then

$$
\lambda_{P}\left(t_{1}, t_{0}\right)=\lambda_{Q}\left(t_{1}, t_{0}, t_{0}, \ldots, t_{0}\right) .
$$

Write $\mathrm{v}_{k}=u_{k} a_{k} m_{k} e$ relative to the Langlands decomposition $Q=\mathcal{U}_{Q} A_{Q}(e) M_{Q}(e)$ and set $a_{k}=\lambda_{Q}\left(t_{1, k}, t_{2, k}, \ldots, t_{q+1, k}\right)$. By (2.4.4) and (2.6.14) we have $f_{\beta}^{Q}\left(y_{k}\right)=$ $t_{1, k} t_{2, k}^{-1} \rightarrow \infty$ where $\beta=\alpha_{m_{1}}$ is the first of these simple roots. It follows from (2.6.7) that, for each of the remaining simple roots $\beta \in \Delta-I, \beta \neq \alpha_{m_{1}}$ we have $f_{\beta}^{H}\left(p_{0}\left(y_{k}\right)\right)=f_{\beta}^{Q}\left(y_{k}\right)$. Hence these also diverge, by hypothesis (2) and Lemma 1.3.6. This completes the verification of condition (b).

2.7. Polyhedral cones. A polyhedral cone $\sigma \subset V$ is a closed convex set,

$$
\sigma=\left\{x \in V \mid \ell_{i}(x) \geq 0 \text { with } i=1,2, \ldots, k\right\}
$$

for some finite collection $\left\{\ell_{1}, \ell_{2}, \ldots, \ell_{k}\right\}$ of linear functions $\ell_{i}: V \rightarrow \mathbb{R}$. The span $L_{\sigma}$ of $\sigma$ is the smallest vector subspace of $V$ which contains $\sigma$. A proper face $\tau$ of $\sigma$ is the intersection of $\sigma$ with a supporting hyperplane (containing the origin). It is again a (closed convex) polyhedral cone. The "interior" $\sigma^{o}$ of $\sigma$ is the complement of its proper faces. The polyhedral cone $\sigma$ is simplicial if $\operatorname{dim}(\sigma)$ equals the number of 1-dimensional faces of $\sigma$.

The vectorspace $V$ is defined over the rationals, $V=\mathbf{V} \otimes_{\mathbb{Q}} \mathbb{R}$. A polyhedral cone $\sigma$ is rational if it is possible to find linear functions $\left\{\ell_{1}, \ell_{2}, \ldots, \ell_{k}\right\}$ defining $\sigma$ which are defined over the rationals. In this case, all the faces of $\sigma$ are rational as well.

LEMMA 2.7.1. ([AMRT] II, 4.3, Thm. 1, p. 113) Any rational polyhedral cone $\sigma \subset C^{*}$ is contained in the closure of a Siegel set. The intersection $\sigma \cap C$ is contained in a Siegel set.

Thus, for any polyhedral cone $\sigma \subset C^{*}$ such that $\sigma^{o} \subset C$ we may identify $\sigma$ with the closure $\overline{\sigma^{o}} \subset \bar{C}^{s t d}$ of $\sigma^{o}$ (in the Satake topology). 
Let $\sigma \subset C^{*}$ be a polyhedral cone. Let $L_{\sigma}$ be the linear subspace of $V$ spanned by $\sigma$ and let $c \in L_{\sigma}^{\perp}$ be an element in the perpendicular complement. Let $\left\{\mathrm{v}_{k}\right\} \subset C$ be a sequence of points, say $\mathrm{v}_{k}=\left(\mathrm{v}_{1, k}, \mathrm{v}_{2, k}\right) \in L_{\sigma}^{\perp} \oplus L_{\sigma}$.

Definition 2.7.2. We say the sequence $\mathrm{v}_{k} \rightarrow c+\infty \sigma$ if

(1) the sequence $\mathrm{v}_{1, k} \rightarrow c$ in $L_{\sigma}^{\perp}$ and

(2) for every $x \in L_{\sigma}$, there exists $N$ so that $k \geq N \Longrightarrow \mathrm{V}_{2, k}-x \in \sigma$.

The following proposition will be needed in $\S 7.9 .2$ and $\S 7.9 .4$, but it is most convenient to prove it here.

Proposition 2.7.3. Let $\left\{\mathrm{v}_{k}\right\} \subset C$ be a sequence and suppose that $\mathrm{v}_{k} \rightarrow c+\infty \sigma$ for some polyhedral cone $\sigma \subset C^{*}$ (with $\sigma^{o} \subset C$ ) and for some $c \in L_{\sigma}^{\perp}$. Suppose the sequence $\left[\mathrm{v}_{k}\right] \in D$ converges in the reductive Borel-Serre compactification to some point $w \in \bar{D}^{R B S}$. Let $\ell \in V$ be any element and suppose $\left\{\ell_{k}\right\} \subset V$ is a sequence which converges to $\ell$. Then for $k$ sufficiently large, the sequence $\mathrm{v}_{k}+\ell_{k}$ is contained in the cone $C$ and its quotient modulo homotheties $\left[\mathrm{v}_{k}+\ell_{k}\right] \in D$ converges in $\bar{D}^{R B S}$ to the same point $w$. (In particular, the sequence $\left[\mathrm{v}_{k}+\ell\right] \rightarrow w$ also.)

Proof 2.7.4. We must show that the sequence $\left[\mathrm{v}_{k}+\ell_{k}\right] \in D$ is contained in a Siegel set and satisfies conditions (1) and (2) of Proposition 2.6.2. The interior $\sigma^{o}$ of the polyhedral cone $\sigma$ may be embedded in the interior $\left(\sigma^{\prime}\right)^{o}$ of a polyhedral cone $\sigma^{\prime} \subset C^{*}$ of top dimension. (There are several ways to do this. If $\sigma$ is a polyhedral cone in a polyhedral decomposition $\Sigma$ of $C$, then $\sigma^{\prime}$ may be taken to be the convex hull of the $\operatorname{star} \operatorname{St}(\sigma)$.) Then $\mathrm{v}_{k} \rightarrow \infty \sigma^{\prime}$. It follows that, for any $\ell \in V$, there exists $N$ so that whenever $k \geq N$ we have $\mathrm{v}_{k}+\ell \in\left(\sigma^{\prime}\right)^{o} \subset C$, so the same is true for the sequence $\mathrm{v}_{k}+\ell_{k}$. By Lemma 2.7.1, the sequence $\mathrm{v}_{k}+\ell_{k}$ is therefore contained in a Siegel set.

The limit point $w$ lies in some RBS boundary component, say, $D_{Q}$. Set $P=P(\epsilon)=Q^{\dagger}$ as in (2.4.15) above. As in (2.4.6) and (2.4.11) the decomposition $L_{P} \cong G_{1} \times G_{0}$ induces $\bar{\Psi}_{P}: \bar{D}_{P}^{R B S} \cong \bar{D}_{1}^{R B S} \times \bar{D}_{0}^{R B S}$ with $\bar{\Psi}_{P}\left(D_{Q}\right)=$ $D_{1} \times D_{0, H}$ by Lemma 2.4.16 (for some rational parabolic subgroup $H \subset G_{0}=$ Aut $\left.^{0}\left(C_{0}(\epsilon), V_{0}(\epsilon)\right)\right)$. Set $\bar{\Psi}_{P}(w)=\left(w_{1}, w_{0}\right)$. Write $\mathrm{v}_{k}=u_{k} a_{k} m_{k}$ relative to the Langlands decomposition of $P$. Then $a_{k}, u_{k}$, and $m_{k}$ are given by matrices (2.6.12), (2.6.13). Since $\left[\mathrm{v}_{k}\right] \rightarrow\left[w_{1}: 0: 0\right]$ the same argument as that following (2.6.13) gives $t_{1, k}^{2} t_{0, k}^{-2} \rightarrow \infty$. Moreover, since $\mathrm{v}_{k} \rightarrow \infty \sigma^{\prime}$ we have

$$
t_{0, k}^{2}\left\langle B_{k}(e-\epsilon), e-\epsilon\right\rangle=\left\langle\mathrm{v}_{k}, e-\epsilon\right\rangle \rightarrow \infty
$$

But $\left\{B_{k}\right\}$ is contained in a compact set of invertible matrices, so we also have $t_{0, k}^{2} \rightarrow \infty$. It follows from (2.6.6) that adding a constant $\ell=\left(\ell_{1}, \ell_{\frac{1}{2}}, \ell_{0}\right)$ will not affect the limiting homothety class. It follows that $\left[\mathrm{v}_{k}+\ell_{k}\right] \rightarrow\left[w_{1}: 0: 0\right]$ in $\bar{D}^{\text {std }}$ which verifies condition (1) of Proposition 2.6.2. 
Condition (2) is verified by induction on the rank of $G$. For sufficiently large $k$ we have

$$
p_{0}\left(\mathrm{v}_{k}\right) \in p_{0}\left(\left(\sigma^{\prime}\right)^{o}\right) \subset C_{0}(\epsilon)
$$

and $\left[p_{0}\left(\mathrm{v}_{k}\right)\right]$ converges in $\bar{D}_{0}^{R B S}$ to the point $w_{0}$. By induction, the sequence $\left[p_{0}\left(\mathrm{v}_{k}\right)+p_{0}\left(\ell_{k}\right)\right] \in D_{0}$ also converges to $\lim \left[p_{0}\left(\mathrm{v}_{k}\right)\right]=w_{0}$ in $\bar{D}_{0}^{R B S}$.

2.8. Blowups of polyhedral cones. Let $\sigma \subset C^{*} \subset V$ be a rational polyhedral cone with $\sigma^{o} \subset C$. Define $\left[\sigma^{o}\right] \subset D$ and $[\sigma] \subset \bar{D}^{\text {std }}$ to be the quotients modulo homotheties. The RBS blowup $[\sigma]^{R B S} \subset \bar{D}^{R B S}$ is the closure of $\left[\sigma^{o}\right] \subset D$ in the reductive Borel-Serre compactification of $D$. The restriction of the mapping $\mu: \bar{D}^{R B S} \rightarrow \bar{D}^{s t d}$ to $[\sigma]^{R B S}$ will be denoted

$$
\mu_{\sigma}:[\sigma]^{R B S} \rightarrow[\sigma]
$$

THEOREM B. Let $C \subset V$ be a rational self adjoint homogeneous cone in a rationally defined real vectorspace. Let $\sigma \subset C^{*}$ be a rational polyhedral cone with $\sigma^{o} \subset C$. Then both $[\sigma]^{R B S}$ and $[\sigma]$ are compact and contractible. Moreover, each admits the structure of a cell complex which is the closure of the single "open" cell $\left[\sigma^{o}\right]$, such that $\mu_{\sigma}:[\sigma]^{R B S} \rightarrow[\sigma]$ is a cellular mapping with contractible fibers.

(In fact, we believe that $[\sigma]^{R B S}$ is homeomorphic to a closed ball.)

First we describe the fibers of the mapping $\mu_{\sigma}$. Let $\sigma \subset C^{*}$ be a rational polyhedral cone with $\sigma^{o} \subset C$. The interior of each face of $\sigma$ is either contained in $C$ or it is contained in some rational boundary component of $C$. Suppose $\tau_{1} \subset \sigma \cap C_{1}(\epsilon)^{*}$ is a proper face of $\sigma$ whose interior $\tau_{1}^{o}$ is contained in the proper rational boundary component $C_{1}(\epsilon)$ with normalizing parabolic subgroup $P=P(\epsilon)$. Let $p_{i}: V \rightarrow V_{i}(\epsilon)$ denote the linear projection (for $i=1,0$ ). Set $D_{i}=C_{i}(\epsilon) /$ homotheties (for $i=1,0$ ) and let $\Psi_{P}: D_{P} \rightarrow D_{1} \times D_{0}$ denote the diffeomorphism of (2.4.18). Define the rational polyhedral cone

$$
\tau_{0}=p_{0}(\sigma) \subset C_{0}(\epsilon)^{*}
$$

LeMma 2.8.3. For any $z \in\left[\tau_{1}^{o}\right] \subset D_{1}$ we have $\mu_{\sigma}^{-1}(z) \subset \bar{D}_{P}^{R B S}$ and

$$
\bar{\Psi}_{P}\left(\left(\mu_{\sigma}\right)^{-1}(z)\right)=\{z\} \times\left[\tau_{0}\right]^{R B S} \subset D_{1} \times \bar{D}_{0}^{R B S}
$$

where $\left[\tau_{0}\right]^{R B S}$ denotes the closure of $\left[\tau_{0}^{o}\right] \subset D_{0}$ in the reductive Borel-Serre compactification of $D_{0}$.

Proof 2.8.4. First let us show that $\left.\bar{\Psi}_{P}\left(\mu_{\sigma}\right)^{-1}(z)\right) \subset\{z\} \times\left[\tau_{0}\right]^{R B S}$. Fix $w \in \mu_{\sigma}^{-1}(z) \subset \bar{D}^{R B S}$. Then $w$ lies in some RBS boundary component $D_{Q}$ for which 
$P=P(\epsilon)=Q^{\dagger}$ (cf. (2.4.15) and Corollary 2.6.3). Put $\bar{\Psi}_{P}(w)=\left(z, w_{0}\right) \in D_{1} \times \bar{D}_{0}^{R B S}$. Since $w \in[\sigma]^{R B S}$, it is a limit of points $y_{k} \in\left[\sigma^{o}\right]$. By Proposition 2.6.2, $y_{k} \rightarrow z$ in $\bar{D}^{\text {std }}$ and the sequence $p_{0}\left(y_{k}\right) \in\left[\tau_{0}^{o}\right]$ converges in $\bar{D}_{0}^{R B S}$ to the point $w_{0}$. This proves that $w_{0} \in\left[\tau_{0}\right]^{R B S}$ as claimed.

Now let us verify the reverse inclusion. We will show that $\bar{\Psi}_{P}\left(\mu_{\sigma}^{-1}(z)\right) \supset$ $\{z\} \times\left[\tau_{0}^{o}\right]$; then the full statement follows from the fact that $\mu_{\sigma}^{-1}(z)$ is a closed subset of $\bar{D}^{R B S}$. So choose $w_{0} \in\left[\tau_{0}^{o}\right]$. Then there is a point $\mathrm{v}_{0} \in \sigma^{o}$ so that $\left[p_{0}\left(\mathrm{~V}_{0}\right)\right]=w_{0}$. Also, choose any lift $z^{\prime} \in C_{1}(\epsilon)$ of $z \in D_{1}$ and let $z_{k} \in \sigma^{o}$ be any sequence so that $z_{k} \rightarrow z^{\prime}$ in the Satake topology of $C^{*}$ and hence also in the usual topology of $V$. Then $p_{0}\left(z_{k}\right) \rightarrow 0$. Now consider the sequence

$$
\mathrm{v}_{k}=\frac{z_{k}}{\sqrt{\left\|p_{0}\left(z_{k}\right)\right\|}}+\mathrm{v}_{0}
$$

Then $\mathrm{v}_{k} \in \sigma^{o}$ because $\sigma^{o}$ is a convex cone; in particular this sequence lies in a Siegel set. Moreover, $\left[\mathrm{v}_{k}\right] \rightarrow z$ in $\bar{D}^{\text {std }}$ because the homothety class is dominated by the first term, while $\left[p_{0}\left(\mathrm{v}_{k}\right)\right] \rightarrow\left[p_{0}\left(\mathrm{v}_{0}\right)\right]=w_{0}$ in $D_{0}$. By Proposition 2.6.2 this implies that the sequence $\left[\mathrm{v}_{k}\right] \in\left[\sigma^{o}\right]$ converges in $\bar{D}^{R B S}$ to the point $\bar{\Psi}_{P}^{-1}\left(z, w_{0}\right)$ as claimed.

2.8.4. Proof of Theorem B. Since $\sigma \subset V$ is a closed convex polyhedral cone, it admits the structure of a subanalytic set, and its quotient modulo homotheties $[\sigma]$ is compact and is subanalytically homeomorphic to a convex polyhedron. The subset $[\sigma]^{R B S}$ also admits the structure of a subanalytic set $[\mathrm{Ha}]$, [Hi] so that the mapping $\mu_{\sigma}:[\sigma]^{R B S} \rightarrow[\sigma]$ is subanalytic. (To see this, it is necessary to check that all the mappings involved in the definition of the topology on $\bar{D}^{R B S}$ are locally subanalytic.) Hence, both sets may be Whitney stratified so that the mapping $\mu_{\sigma}$ is a "weakly" stratified map.

Let $z \in[\sigma]$. Then $z$ lies in the interior $\left[\tau_{1}^{o}\right]$ of some face $\left[\tau_{1}\right] \subset[\sigma]$. If $\tau_{1}^{o} \subset C$ then the fiber $\mu_{\sigma}^{-1}(z)$ consists of a single point. Otherwise, $\tau_{1}^{o}$ lies in some proper boundary component $C_{1}(\epsilon)$, in which case the fiber $\mu_{\sigma}^{-1}(z)$ has been identified by Lemma 2.8.3 with a certain subset $\left[\tau_{0}\right]^{R B S}$ (which is the closure in $\bar{D}_{0}^{R B S}$ of the interior $\left[\tau_{0}^{o}\right]$ of a certain polyhedral cone $\tau_{0}$ modulo homotheties). By induction, this fiber is compact and contractible. It follows that the mapping $\mu_{\sigma}$ is proper, that $[\sigma]^{R B S}$ is compact, and by Proposition 8.2 it is contractible.

Finally, we sketch a proof that $[\sigma]^{R B S}$ admits the structure of a cell complex, although it will not be needed in this paper. First there is a "paving" of $[\sigma]^{R B S}$ by "open" subanalytic cells. If $\tau_{1}$ is a face of $\sigma$ then either $\tau_{1}^{o} \subset C$ (in which case $\mu_{\sigma}^{-1}\left(\left[\tau_{1}^{o}\right]\right) \cong \tau_{1}^{o}$ is a cell) or else $\tau_{1}^{o}$ lies in some proper boundary component $C_{1}(\epsilon)$, in which case $\mu_{\sigma}^{-1}\left(\tau_{1}^{o}\right) \cong \tau_{1}^{o} \times\left[\tau_{0}\right]^{R B S}$ which is, by induction, paved by cells of the form $\tau_{1}^{o} \times$ cell of $\left[\tau_{0}\right]^{R B S}$. It can be shown that the closure of each of these cells is a subanalytic set. But it follows from stratification theory that whenever $W$ is a compact subanalytic set which is paved by subanalytic cells, 
then these are in fact the cells of a cell complex: the attaching maps may be constructed from Thom-Mather tubular neighborhood data. (Note that we have not shown that this decomposition of $[\sigma]^{R B S}$ is a regular cell decomposition, although we believe this also to be true.)

3. Admissible polyhedral decompositions. In this section we define the notion of a "sufficiently fine $\Gamma$-admissible polyhedral decomposition" $\Sigma$ of a selfadjoint homogeneous cone $C$, and we show (Theorem 3.7) that they are cofinal in the collection of all $\Gamma$-admissible polyhedral decompositions of $C$.

3.1. As in $\S 2$, we suppose that $C \subset V=\mathbf{V} \otimes_{\mathbb{Q}} \mathbb{R}$ is a rationally defined self adjoint homogeneous cone with automorphism group $G=\mathbf{G}(\mathbb{R})$, and quotient under homotheties $D=[C]$. Let $C^{*} \subset V$ denote the union of $C$ and all its rational boundary components, and let $\bar{D}^{\text {std }}$ denote its quotient under homotheties, with the Satake topology. Let $\Gamma \subset \mathbf{G}(\mathbb{Q})$ be a neat arithmetic group. Let $\Sigma=\{\sigma\}$ be a collection of rational polyhedral cones $\sigma \subset C^{*}$. The collection $\Sigma$ is a polyhedral cone decomposition of $C^{*}$ provided that:

(1) Each $\sigma$ is a closed polyhedral cone in the (rationally defined) vectorspace spanned by the 1-dimensional faces of $\sigma_{\alpha}$.

(2) If $\sigma \in \Sigma$ then each face $\tau$ of $\sigma$ is also an element of $\Sigma$.

(3) Each intersection $\sigma \cap \tau$ is $\{0\}$ or else it is a common face of each.

(4) The cone $C^{*}$ is the disjoint union

$$
C^{*}=\coprod_{\sigma \in \Sigma} \sigma^{o}
$$

of the interiors of the cones in $\Sigma$.

Such a polyhedral decomposition is $\Gamma$-admissible provided that:

(1) For all $\gamma \in \Gamma$ and for any polyhedral cone $\sigma \in \Sigma$, we have $\gamma \sigma \in \Sigma$.

(2) The collection $\{\sigma\} / \Gamma$ is finite.

Let us say that a closed subset $S \subset \bar{D}^{\text {std }}$ is $\Gamma$-small if, for each $\gamma \in \Gamma$, either $S \cap \gamma S=\phi$ or $\gamma$ acts as the identity on $S \cap \gamma S$. A polyhedral cone $\sigma \subset C^{*}$ is $\Gamma$ small if its homothetic quotient $[\sigma] \subset \bar{D}^{s t d}$ is $\Gamma$-small. The cone decomposition $\Sigma$ is $\Gamma$-fine if every cone $\sigma \in \Sigma$ is $\Gamma$-small. Let us say that a closed subset $S \subset \bar{D}^{R B S}$ is $\Gamma$-sufficiently small if, for each $\gamma \in \Gamma$, either $S \cap \gamma S=\phi$ or else $\gamma$ acts as the identity on $S \cap \gamma S$. A polyhedral cone $\sigma \subset C^{*}$ with $\sigma^{o} \subset C$ is $\Gamma$-sufficiently small if the closed subset $[\sigma]^{R B S}$ is $\Gamma$-sufficiently small. A polyhedral cone $\sigma \subset C^{*}$ with $\sigma^{o} \subset C_{1}(\epsilon)$ is $\Gamma$-sufficiently small if the closed subset $[\sigma]^{R B S} \subset{\overline{\left[C_{1}(\epsilon)\right.}}^{R B S}$ is $\Gamma_{P^{-}}$-sufficiently small (where $\Gamma_{P}=\Gamma \cap P$ is the intersection of $\Gamma$ with the parabolic subgroup $P$ which preserves the boundary component $C_{1}(\epsilon)$ ). The cone decomposition $\Sigma$ is $\Gamma$-sufficiently fine if every cone $\sigma_{\alpha} \in \Sigma$ is $\Gamma$-sufficiently small. 
(So the words "small" and "fine" refer to the standard compactification; the words "sufficiently small" and "sufficiently fine" refer to the RBS compactification.)

If $\pi: \bar{C}^{s t d} \rightarrow \Gamma \backslash \bar{C}^{\text {std }}$ denotes the quotient mapping and if $\sigma \subset C^{*}$ is $\Gamma$-small, then $\pi \mid \sigma$ is a homeomorphism onto its image; in other words the quotient under $\Gamma$ does not introduce any identifications on $\sigma$. Hence, a $\Gamma$-fine polyhedral decomposition of $C^{*}$ induces a ("flat") regular cell decomposition of the standard compactification $\overline{\Gamma \backslash D}^{\text {std }}=\Gamma \backslash C^{*} / A_{G}$. If the polyhedral decomposition $\Sigma$ is simplicial (meaning that it consists of simplicial cones), then the induced regular cell decomposition of $\overline{\Gamma \backslash D}^{\text {std }}$ is a ("flat") triangulation.

Lemma 3.2. Suppose $\sigma \subset C^{*}$ is a closed polyhedral cone. Let $\gamma \in \Gamma$ and suppose that $\gamma \sigma=\sigma$. Then $\gamma$ acts as the identity on $\sigma$.

Proof 3.3. The interior of $\sigma$ is contained in some boundary component $C^{\prime}$ (possibly $C^{\prime}=C$ ). Let $P=\mathcal{U}_{P} G_{1} G_{0}$ denote the maximal parabolic subgroup which normalizes $C^{\prime}$ where $G_{1}=\operatorname{Aut}^{0}\left(C^{\prime}\right)$. Let $\nu_{1}: P \rightarrow G_{1}$ denote the projection. Then the element $\gamma$ acts on $\sigma$ through its projection $\nu_{1}(\gamma) \subset \Gamma^{\prime}=\nu_{1}(\Gamma)$. Consider the subgroup

$$
\Gamma_{\sigma}^{\prime}=\left\{\gamma^{\prime} \in \Gamma^{\prime} \mid \gamma^{\prime} \sigma=\sigma\right\}
$$

This group is finite since it is contained in the subset

$$
\left\{\gamma^{\prime} \in \Gamma^{\prime} \mid \gamma^{\prime} \sigma \cap \sigma \cap C^{\prime} \neq \phi\right\}
$$

(which is finite by Lemma 2.7.1). Since $\Gamma$ is neat, we conclude that $\Gamma_{\sigma}^{\prime}=\{1\}$ so $\gamma \in \operatorname{ker} \nu_{1}=Z\left(C^{\prime}\right)$. In other words, $\gamma$ acts trivially on $C^{\prime}$, hence also on $\sigma$.

3.4. Refinements. If $\Sigma$ is a (rational) polyhedral cone decomposition of $C^{*}$, a (rational) first barycentric subdivision of $\Sigma$ is determined by a choice of (rational) 1-dimensional cone (which is usually called "a barycenter") $\hat{\sigma} \in \sigma^{o}$ in the interior of each cone $\sigma \in \Sigma$, and consists of simplicial cones which are spanned by 1 -dimensional cones $\widehat{\sigma}_{1}, \widehat{\sigma}_{2}, \ldots, \widehat{\sigma}_{k}$ for each chain $\sigma_{1} \subset \sigma_{2} \subset \ldots \subset \sigma_{k}$ in $\Sigma$.

If $\Sigma$ is a $\Gamma$-admissible polyhedral cone decomposition of $C^{*}$ and if $\Sigma^{\prime}$ is a (rational) first barycentric subdivision of $\Sigma$ then $\Sigma^{\prime}$ is $\Gamma$-admissible iff the choices $\widehat{\sigma}$ are $\Gamma$-compatible, i.e., for all $\gamma \in \Gamma$ and for all $\sigma \in \Sigma$ we have $\widehat{\gamma \sigma}=\gamma \widehat{\sigma}$. If $\Sigma$ is $\Gamma$-admissible then by Lemma 3.2 there exists a $\Gamma$-compatible set of choices of (rational) barycenters.

Lemma 3.5. Suppose $\Sigma$ is a $\Gamma$-admissible polyhedral decomposition of $C^{*}$. Let $L$ be a closed subcomplex of $\Sigma$ such that its support $|L| \subset C^{*}$ is $\Gamma$-small. Let $L^{\prime}$ be a choice of first (rational) barycentric subdivision of $L$. Then there is a choice 
of first (rational) barycentric subdivision $\Sigma^{\prime}$ of $\Sigma$ which is $\Gamma$-admissible and which contains $L^{\prime}$ as a subcomplex.

Proof 3.6. For each cone $\sigma \in L$ and for each $\gamma \in \Gamma$ the choice of barycenter $\hat{\sigma}$ of $\sigma$ determines a unique choice of barycenter $\gamma \hat{\sigma}$ of $\gamma \sigma$ because $|L|$ is $\Gamma$ small. Modulo $\Gamma$, there are finitely many remaining cones $\sigma \notin \Gamma \cdot L$. Choose a single representative cone $\sigma$ from each equivalence class, choose its barycenter $\widehat{\sigma}$ arbitrarily, and translate by $\Gamma$. By Lemma (3.2), this gives a well-defined $\Gamma$ invariant family of barycenters, so the resulting first barycentric subdivision of $\Sigma$ is $\Gamma$-admissible.

THEOREM 3.7. Let $\Sigma$ be a rational cone decomposition of $C^{*}$. Let $\Gamma \subset \mathbf{G}(\mathbb{Q})$ be a neat arithmetic group.

(1) If $\Sigma$ is $\Gamma$-sufficiently fine, then $\Sigma$ is $\Gamma$-fine.

(2) If $\Sigma$ is a $\Gamma$-admissible (resp. $\Gamma$-fine, resp. $\Gamma$-sufficiently fine) decomposition of $C^{*}$ and if $\Gamma^{\prime} \subset \Gamma$ is an arithmetic subgroup, then $\Sigma$ is a $\Gamma^{\prime}$-admissible (resp. $\Gamma^{\prime}$-fine, resp. $\Gamma^{\prime}$-sufficiently fine) decomposition of $C^{*}$.

(3) Suppose $\Sigma$ is a $\Gamma$-admissible decomposition of $C^{*}$, and $\Sigma^{\prime}$ is a $\Gamma$-admissible refinement of $\Sigma$. If $\Sigma$ is $\Gamma$-fine (resp. $\Gamma$-sufficiently fine) then $\Sigma^{\prime}$ is also $\Gamma$-fine (resp. $\Gamma$-sufficiently fine).

(4) If $\Sigma$ is $\Gamma$-admissible, then there is a refinement $\Sigma^{\prime}$ of $\Sigma$ which is $\Gamma$-fine.

(5) If $\Sigma$ is $\Gamma$-fine, then there is a refinement $\Sigma^{\prime}$ of $\Sigma$ which is $\Gamma$-sufficiently fine.

Proof 3.8. Part (1) follows from the fact that the mapping $\mu: \bar{D}^{R B S} \rightarrow$ $\bar{D}^{\text {std }}$ commutes with the action of $\Gamma$. Parts (2) and (3) follow directly from the definitions.

Let us consider part (4). We shall prove that any rational first barycentric subdivision of any $\Gamma$-admissible decomposition $\Sigma$ is a $\Gamma$-fine decomposition.

Let $\Sigma$ be a $\Gamma$-admissible polyhedral decomposition of $C^{*}$ and let $\Sigma^{\prime}$ be a $\Gamma$-admissible first barycentric subdivision of $\Sigma$. Let $\tau \in \Sigma^{\prime}$ be a simplicial cone in the first barycentric subdivision of some simplicial cone $\sigma$. We claim that $\tau$ is $\Gamma$-small.

Write $\tau=\left\langle\hat{s}_{0} \hat{s}_{1} \ldots \hat{s}_{r}\right\rangle$ where $s_{0}<s_{1}<\cdots<s_{r}$ are faces of $\sigma$ (and where $\left\langle\mathrm{v}_{0} \mathrm{v}_{1} \ldots \mathrm{V}_{r}\right\rangle$ denotes the simplicial cone spanned by 1-dimensional cones $\left.\mathrm{v}_{0}, \mathrm{v}_{1}, \ldots, \mathrm{v}_{r}\right)$. So

$$
\gamma \tau=\left\langle\left(\gamma \hat{s}_{0}\right)\left(\gamma \hat{\boldsymbol{S}}_{1}\right) \cdots\left(\gamma \hat{\boldsymbol{S}}_{r}\right)\right\rangle
$$

Since $\gamma$ acts linearly and takes 1-dimensional cones of $\Sigma^{\prime}$ to 1-dimensional cones of $\Sigma^{\prime}$, the intersection $\tau \cap \gamma \tau$ is precisely the simplex which is spanned by the 1dimensional cones which are in common among the two sets $\left\{\hat{s}_{0}, \hat{s}_{1}, \ldots, \hat{s}_{r}\right\}$ and $\left\{\left(\gamma \hat{s}_{0}\right),\left(\gamma \hat{s}_{1}\right), \ldots,\left(\gamma \hat{s}_{r}\right)\right\}$. However, if $\hat{s}_{i}=\gamma \hat{s}_{j}$ then $s_{i}=\gamma s_{j}$ and $\operatorname{dim}\left(s_{i}\right)=\operatorname{dim}\left(s_{j}\right)$. 
Since the chain $s_{0}<s_{1}<\cdots<s_{r}$ is strictly increasing we conclude that $s_{i}=s_{j}$. In other words, any 1-dimensional cone $\hat{s}_{i}=\gamma \hat{s}_{j}$ which is in common between these two sets is actually preserved by $\gamma$. Therefore, $\tau \cap \gamma \tau$ is spanned by 1dimensional cones, each of which is preserved by $\gamma$. The last 1-dimensional cone $\hat{s}_{r}$ lies in (the interior of) the boundary component $C^{\prime}$ whose closure contains $\tau$. Since $\Gamma$ is neat, and $\gamma$ preserves $\hat{s}_{r}$ it follows (as in the proof of Lemma 3.2) that $\gamma$ acts as the identity on $C^{\prime}$ and hence it acts as the identity on $\tau$. This completes the proof that $\tau$ is $\Gamma$-small.

3.9. The rest of $\S 3$ is devoted to proving part (5) of Theorem 3.7. Let us say that a polyhedral cone $\sigma \subset C$ is flag like if its intersection with the rational boundary components of $C$ form a flag, i.e., if there is a partial flag of boundary components

$$
C_{1} \subset \overline{C_{2}} \subset \cdots \subset \overline{C_{m}}
$$

and a (partial) chain of faces

$$
\tau_{1} \subset \tau_{2} \subset \cdots \subset \tau_{m}=\sigma
$$

such that for each $i$ (with $1 \leq i \leq m$ ), the interior of $\tau_{i}$ is contained in $C_{i}$ and the intersection $\tau_{i} \cap \partial C_{i}^{*}=\tau_{i-1}$ where $\partial C_{i}^{*}=C_{i}^{*}-C_{i}$ denotes the proper rational boundary components of $C_{i}$. If $\sigma \in \Sigma$ is a flag-like polyhedral cone with associated partial flag (3.9.1), then there are associated rational idempotents $d_{1}, d_{2}, \ldots, d_{m}$ so that $C_{1}=C_{1}\left(d_{1}\right), C_{2}=C_{1}\left(d_{1}+d_{2}\right), \ldots, C_{m}=C_{1}\left(d_{1}+\cdots+d_{m}\right)$. Let $p_{0}^{(i)}: C_{d} \rightarrow C_{0}\left(d_{1}+d_{2}+\cdots+d_{i}\right)$ and $p_{1}^{(i)}: C_{d} \rightarrow C_{1}\left(d_{1}+d_{2}+\cdots+d_{i}\right)$ denote the linear projections which are determined by the Peirce decompositions for these idempotents.

LEMMA 3.10. If $\sigma$ is $\Gamma$-small and flag-like and if each for each $i$ (with $1 \leq i \leq$ $m-1)$ the image

$$
p_{0}^{(i)}(\sigma) \subset C_{0}\left(d_{1}+\cdots+d_{i}\right)^{*} \text { is } \Gamma \text {-small }
$$

then $\sigma$ is $\Gamma$-sufficiently small.

The proof is by induction on the $\mathbb{Q}$-rank of the linear symmetric space $C_{m}$ which contains $\sigma$ (with the rank 0 case being trivial). For notational convenience, let us assume that $\sigma \subset C=C_{m}$. Let $\gamma \in \Gamma$ and suppose that $\sigma$ satisfies (3.10.1). Let $x \in[\sigma]^{R B S} \cap \gamma[\sigma]^{R B S}$. We must show that $\gamma x=x$. Let $\mu: \bar{D}^{R B S} \rightarrow \bar{D}^{\text {std }}$ denote the projection. Then $x_{1}:=\mu(x)$ lies in some rational boundary component which is associated to $\sigma$, say

$$
x_{1}=\mu(x) \in\left[C_{1}(\epsilon)\right]
$$


where $\epsilon=d_{1}+d_{2}+\cdots+d_{k}$ for some $k, 1 \leq k \leq m$. Since $\mu$ commutes with the action of $\Gamma$, we have $x_{1}=\mu(x) \in \sigma \cap \gamma \sigma$ hence (since $\sigma$ is $\Gamma$-small),

$$
\gamma x_{1}=x_{1}
$$

It also follows that $\gamma \in \Gamma_{P}=\Gamma \cap P$ where $P$ denotes the maximal parabolic subgroup which normalizes this boundary component. (Hence $x \in \bar{D}_{P}^{R B S}$.)

Recall (§2.4) that the Levi quotient splits as an almost direct product, $L_{P} \cong$ $G_{1} \times G_{0}$ where $G_{i}=\operatorname{Aut}^{0}\left(C_{i}(\epsilon)\right)$, and that this splitting induces a homeomorphism

$$
\bar{D}_{P}^{R B S} \cong \bar{D}_{1}^{R B S} \times \bar{D}_{0}^{R B S}
$$

(where $D_{i}$ is the linear symmetric space associated with the self adjoint homogeneous cone $\left.C_{i}(\epsilon), i=0,1\right)$. Write $x=\left(x_{1}, x_{0}\right)$ relative to this product decomposition. The element $\gamma$ acts on the RBS boundary component $D_{P}$ via its projection, $\nu_{P}(\gamma)=\left(\gamma_{1}, \gamma_{2}\right) \in L_{P}$ to the Levi quotient. In summary,

$$
\gamma x=\left(\gamma_{1} x_{1}, \gamma_{0} x_{0}\right) \in D_{1} \times \bar{D}_{0}^{R B S}
$$

By (3.10.2), since $\Gamma$ is neat, we have $\gamma_{1}=1$. Therefore $\gamma$ preserves the fiber $\mu^{-1}\left(x_{1}\right)=\left\{x_{1}\right\} \times\left[\tau_{0}\right]^{R B S}$ (by $\S 2.4$ ) where

$$
\tau_{0}=p_{0}(\sigma) \subset C_{0}(\epsilon)^{*}
$$

(For simplicity we write $p_{0}$ and $p_{1}$ rather than $p_{0}^{(k)}$ and $p_{1}^{(k)}$.) But $x_{0} \in\left[\tau_{0}\right]^{R B S} \cap$ $\gamma_{0}\left[\tau_{0}\right]^{R B S}$. The assumption (3.10.1) is that $\tau_{0}$ is $\Gamma$-small. Since $\tau_{0}$ is contained in the lower rank symmetric space $C_{0}(\epsilon)$, it follows from the induction hypothesis that $\gamma_{0} x_{0}=x_{0}$. In summary, $\gamma x=\left(\gamma_{1} x_{1}, \gamma_{0} x_{0}\right)=\left(x_{1}, x_{0}\right)=x$ as claimed.

3.11. If $\sigma \subset C^{*}$ is a closed polyhedral cone, then any first barycentric subdivision of $\sigma$ is both flag-like and simplicial. If $\sigma \in \Sigma$ is flag-like with respect to a chain of boundary components $C_{1} \subset \bar{C}_{2} \subset \cdots \subset \bar{C}_{k}$ then any polyhedral cone $\tau$ in any first barycentric subdivision of $\sigma$ is flag-like with respect to some sub-chain $C_{i_{1}} \subset \bar{C}_{i_{2}} \subset \cdots \subset \bar{C}_{i_{r}}$. Let us say that a (closed) polyhedral cone $\sigma \subset C^{*}$ is compatible with $\Gamma$ if, for each $\gamma \in \Gamma$, either $\sigma \cap \gamma \sigma=\phi$ or else it is a face of $\sigma$. If $\sigma \subset C^{*}$ is compatible with $\Gamma$, then (as in the proof of part(4) of Theorem 3.7) its first barycentric subdivision consists of small cells.

Lemma 3.12. Suppose $\sigma \subset C^{*}$ is a rational (closed) polyhedral cone. Then it admits a rational refinement, each of whose polyhedral cones is compatible with $\Gamma$.

Proof 3.13. By taking a rational first barycentric subdivision if necessary, we may assume that $\sigma$ is flag-like with respect to some chain $C_{1} \subset \bar{C}_{2} \subset \cdots \subset \bar{C}_{m}$ 
of rational boundary components. For $i=1,2, \ldots, m$ let $P_{i}=P\left(C_{i}\right)$ denote the corresponding normalizing maximal parabolic subgroups and define

$$
\Gamma_{\sigma, i}=\left\{\gamma \in \Gamma \mid \gamma \sigma \cap \sigma \cap C_{i} \neq \phi \text { and } \gamma \sigma \cap \sigma \cap C_{j}=\phi \text { for all } j>i\right\} .
$$

The flag-like assumption on $\sigma$ guarantees that $\Gamma_{\sigma, i} \subset P_{i}$. (Since the $C_{i}$ form a chain of boundary components, for any $\gamma \in \Gamma$ and for any $i \neq j$ we have $\gamma C_{i} \cap C_{j}=\phi$. Since $\sigma$ is flag-like, it is the disjoint union of the subsets $\sigma \cap C_{j}$. So, if $\gamma \in \Gamma_{\sigma, i}$ then $\gamma\left(\sigma \cap C_{i}\right) \cap\left(\sigma \cap C_{i}\right) \neq \phi$. Hence $\gamma C_{i} \cap C_{i} \neq \phi$ so $\gamma \in P\left(C_{i}\right)$.)

The discrete group $\Gamma_{i}^{\prime}=\nu_{i}\left(\Gamma \cap P_{i}\right)$ acts on the boundary component $C_{i}$, where $\nu_{i}$ is the composite projection

$$
P_{i} \rightarrow L_{P_{i}}=G_{1, i} G_{0, i} \rightarrow G_{1, i} /\left(G_{1, i} \cap G_{0, i}\right) .
$$

By [AMRT] II $\S 4.3$ p. 116, the set

$$
\Gamma_{\sigma, i}^{\prime}=\nu_{i}\left(\Gamma_{\sigma, i}\right) \subset \Gamma_{i}^{\prime}
$$

is finite: it is a subset of $\left\{\tau \in \Gamma_{i}^{\prime} \mid \tau\left(\sigma \cap C_{i}\right) \cap\left(\sigma \cap C_{i}\right) \neq \phi\right\}$ which is finite. Let $\Gamma_{\sigma, i}^{\prime \prime} \subset \Gamma_{\sigma, i}$ be a set of lifts of these finitely many elements. Define $\Gamma_{\sigma}^{\prime \prime}=\cup_{i=1}^{m} \Gamma_{\sigma, i}^{\prime \prime}$. This is a finite set of elements which completely captures the possible nontrivial intersections $\sigma \cap \gamma \sigma$ for $\gamma \in \Gamma$. As in [AMRT] II $\S 4.3$, choose finitely many rational hyperplanes $H_{1}, H_{2}, \ldots, H_{m}$ which define $\sigma$ (and its faces), i.e., so that $L_{\sigma}=H_{1} \cap H_{2} \cap \cdots \cap H_{k}$ and so that $\sigma=L_{\sigma} \cap H_{k+1}^{+} \cap H_{k+2}^{+} \ldots \cap H_{m}^{+}$(where $H_{j}^{+}$ denotes a chosen halfspace on one side of the hyperplane $H_{j}$ ). Then the connected components of the complement

$$
\sigma-\bigcup\left\{\gamma H_{j} \mid \gamma \in \Gamma_{\sigma}^{\prime \prime} \text { and } 1 \leq j \leq m\right\}
$$

(and all their faces) form a $\Gamma$-compatible refinement of $\sigma$.

COROLlaRY 3.14. Let $\sigma \subset C^{*}$ be a closed rational polyhedral cone. Then $\sigma$ has a rational refinement, each of whose cones are $\Gamma$-small.

Proof 3.15. First choose a rational refinement which is compatible with $\Gamma$. Now choose a rational $\Gamma$-invariant first barycentric subdivision of that. Now the same argument as in the proof of part (4) of Theorem 3.7 shows that each cell in this barycentric subdivision is $\Gamma$-small.

3.16. Proof of part (5). We will need to use the following lemmas from P.L. topology (e.g. [Hu] $\S 3$, Cor. 1.6; $\S 4,1.8$; and $\S 4,1.9$ ):

(1) If $K$ and $L$ are simplicial complexes and if $|K| \subset|L|$ then for some $r$ there exists an $r$ th barycentric subdivision $L^{(r)}$ of $L$ which contains a subdivision of $K$. 
(2) Let $K$ and $L$ be simplicial complexes and $f: K \rightarrow L$ a simplicial mapping. Given any subdivision $L^{\prime}$ of $L$ there exists a subdivision $K^{\prime}$ of $K$ so that $f: K^{\prime} \rightarrow L^{\prime}$ is simplicial.

(3) Let $K$ and $L$ be simplicial complexes, and $f:|K| \rightarrow|L|$ a continuous mapping whose restriction to each cell of $K$ is linear. Then there are subdivisions $K^{\prime}$ of $K$ and $L^{\prime}$ of $L$ so that $f: K^{\prime} \rightarrow L^{\prime}$ is simplicial.

If $\sigma \subset C^{*}$ is a flag-like polyhedral cone with respect to a flag (3.9.1) of boundary components, we will say that the resulting projections $p_{0}^{(i)}: \sigma \rightarrow C_{0}\left(d_{1}+\right.$ $\cdots+d_{i}$ ) (for $1 \leq i \leq m-1$ ) are relevant for $\sigma$. If $\sigma$ is $\Gamma$-small and flag-like and if, for each relevant projection $p$ the image $p(\sigma)$ is $\Gamma$-small, then the same is true for every translate $\gamma \sigma$ (for any $\gamma \in \Gamma$ ).

Now suppose that $\Sigma$ is a $\Gamma$-fine, flag-like decomposition of $C^{*}$. Modulo $\Gamma$, there are finitely many pairs $(\sigma, p)$ where $\sigma \in \Sigma$ and where $p$ is a relevant projection for $\sigma$. Order a collection of unique representatives (modulo $\Gamma$ ) of these pairs in any way, $\left(\sigma_{1}, p_{1}\right),\left(\sigma_{2}, p_{2}\right), \ldots,\left(\sigma_{n}, p_{n}\right)$ (so a given polyhedral cone may be repeated many times in this ordering). Let us suppose by induction that we have found a refinement $\Sigma^{\prime}$ of $\Sigma$ with the following property:

$\left(P_{m-1}\right)$ Whenever $\eta \in \Sigma^{\prime}$ is a polyhedral cone which is contained in some $\sigma_{i}$ (where $1 \leq i \leq m-1$ ) then $p_{i}(\eta)$ is $\Gamma$-small.

Now let us further refine $\Sigma^{\prime}$ so that the same holds for all polyhedral cones contained in $\sigma_{m}$. Let $K \subset \Sigma^{\prime}$ be the simplicial complex consisting of all simplices in $\sigma_{m}$. Let $L$ be the simplicial complex consisting of the polyhedral cone $p_{m}\left(\sigma_{m}\right)$ together with all its faces. By (3) above, there are subdivisions $K^{\prime}$ of $K$ and $L^{\prime}$ of $L$ so that the projection $p_{m}: K^{\prime} \rightarrow L^{\prime}$ is simplicial. By Corollary 3.14 there is a different subdivision $L^{\prime \prime}$ of $L$ so that the cones in $L^{\prime \prime}$ are $\Gamma$-small. Let $L^{\prime \prime \prime}$ be the common refinement of $L^{\prime}$ and $L^{\prime \prime}$. By (2) above, there is a subdivision (let us call it $K^{\prime \prime \prime}$ ) of $K^{\prime}$ so that $p_{m}: K^{\prime \prime \prime} \rightarrow L^{\prime \prime \prime}$ is simplicial. By (1) above, there is a barycentric refinement $K^{(r)}$ which contains a subdivision of $K^{\prime \prime \prime}$ as a subcomplex. By Lemma 3.5 this barycentric refinement may be extended to a $\Gamma$-invariant barycentric refinement $\Sigma^{(r)}$ of $\Sigma$. Since the property of being $\Gamma$-small is inherited by closed subsets, every simplex in $\Sigma^{(r)}$ whose support is contained in $\sigma_{1} \cup \cdots \cup \sigma_{m}$ is small and all of their relevant projections are also small. This verifies condition $\left(P_{m}\right)$ above and completes the inductive step. In summary, it is possible to find a refinement consisting of simplices which are $\Gamma$-small, and for which every relevant projection is $\Gamma$-small. This means that each simplex is $\Gamma$-sufficiently small.

4. Hermitian symmetric spaces. The main result in this chapter is Theorem $\mathrm{C}(\S 4.2)$. This, together with Theorem B $(\S 2.8)$ are the main technical results which are needed for the proof of Theorem A. 
Throughout the rest of the paper, we suppose that $\mathbf{G}$ is semisimple over $\mathbb{Q}$ and that the associated symmetric space $D=G / K$ is Hermitian. (This disagrees with our previous use of the symbol $D$ in $\S 2$ and $\S 3$.) We may assume that $D$ is a bounded symmetric domain in some $\mathbb{C}^{N}$. Denote by $\bar{D}$ its closure in $\mathbb{C}^{N}$; it is a disjoint union of boundary components. The action of $G$ on $D$ extends continuously to the closure $\bar{D}$. As above, let us fix a basepoint $x_{0} \in D$ and a standard minimal rational parabolic subgroup $\mathbf{Q}_{0} \in \mathbf{G}$.

4.1. Suppose $F \subset \bar{D}$ is a rational boundary component. Let $\mathbf{P} \subset \mathbf{G}$ be its normalizing subgroup: it is a maximal rational parabolic subgroup of $\mathbf{G}$. After conjugating by some element of $\mathbf{G}(\mathbb{Q})$ (if necessary), we may assume that $\mathbf{P}$ is standard, i.e., $\mathbf{P} \supset \mathbf{Q}_{0}$. The Levi quotient $L_{P}=P / \mathcal{U}_{P}$ splits as an almost direct product $L_{P}=G_{h} G_{\ell}$ where $G_{h}$ acts transitively on $F$, and where $G_{\ell}$ acts transitively on a certain self adjoint homogeneous cone $C_{P} \subset \mathfrak{z}$. (Here, $\mathfrak{z} \subset \mathfrak{u}_{P}$ is the center of the Lie algebra $\mathfrak{u}_{P}$ of the unipotent radical $\mathcal{U}_{P}$ of $P$.) Furthermore, $A_{P} \subset G_{\ell}$. The choice of basepoint $x_{0} \in D$ determines basepoints $z_{0} \in F, e \in C_{P}$; it determines maximal compact subgroups $K_{h}=K \cap G_{h}\left(x_{0}\right)=\operatorname{Stab}_{G_{h}}\left(z_{0}\right) \subset G_{h}$, $K_{\ell}=K \cap G_{\ell}\left(x_{0}\right)=\operatorname{Stab}_{G_{\ell}}(e)$; and it determines diffeomorphisms $G_{h} / K_{h} \cong F$, $G_{\ell} / K_{\ell} \cong C_{P}$. The mapping

$$
\psi:\left(G_{h} / K_{h}\right) \times\left(G_{\ell} / K_{\ell}\right)=F \times C_{P} \rightarrow L_{P} / K_{P} \cong P / K_{P} \mathcal{U}_{P}
$$

given by

$$
\left(g_{h} K_{h}, g_{\ell} K_{\ell}\right) \mapsto g_{h} g_{\ell} K_{P}
$$

is a diffeomorphism.

Let $\left[C_{P}\right]$ denote the quotient of $C_{P}$ under the torus of homotheties; hence $\left[C_{P}\right] \cong G_{\ell} / K_{\ell} A_{P}$, and let $D_{P}=P / K_{P} A_{P} \mathcal{U}_{P}$ denote the boundary component of the reductive Borel-Serre partial compactification $\bar{D}^{R B S}$ corresponding to the parabolic subgroup $P$. Then the above diffeomorphism $\psi$ induces a diffeomorphism

$$
\Psi_{P}: F \times\left[C_{P}\right] \stackrel{\cong}{\longrightarrow} P / K_{P} A_{P} \mathcal{U}_{P}=D_{P}
$$

which extends to a stratum preserving homeomorphism (which is smooth on each stratum),

$$
\bar{\Psi}_{P}: \bar{F}^{R B S} \times{\overline{\left[C_{P}\right]}}^{R B S} \stackrel{\cong}{\longrightarrow} \bar{D}_{P}^{R B S} \subset \bar{D}^{R B S}
$$

on the reductive Borel-Serre partial compactifications. Composing the canonical projection $D=P / K_{P} \rightarrow P / K_{P} \mathcal{U}_{P}$ with the diffeomorphism $\psi^{-1}$ and with projection to the two factors $F$ and $C_{P}$, defines smooth mappings $\Phi_{h}: D \rightarrow F$, 
$\phi_{\ell}: D \rightarrow C_{P}$, and $\Phi_{\ell}: D \rightarrow\left[C_{P}\right]$, i.e.,

$$
\begin{aligned}
\Phi_{h}\left(g K_{P}\right) & =g K_{P} \mathcal{U}_{P} G_{\ell} \in P / K_{P} \mathcal{U}_{P} G_{\ell} \cong F \\
\phi_{\ell}\left(g K_{P}\right) & =g K_{P} \mathcal{U}_{P} G_{h} \in P / K_{P} \mathcal{U}_{P} G_{h} \cong C_{P} \\
\Phi_{\ell}\left(g K_{P}\right) & =g K_{P} A_{P} \mathcal{U}_{P} G_{h} \in P / K_{P} A_{P} \mathcal{U}_{P} G_{h} \cong\left[C_{P}\right]
\end{aligned}
$$

for any $g K_{P} \in P / K_{P} \cong D$. Then the following diagram commutes; the composition across the top row is $\phi_{\ell}$ and the composition across the bottom row is $\Phi_{\ell}$,

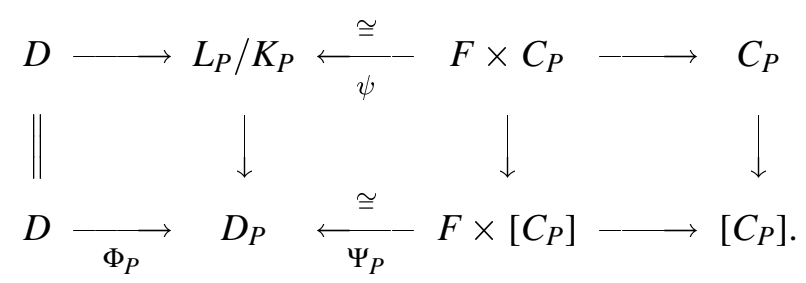

If $\left\{y_{k}\right\} \subset C_{P} \subset \mathfrak{z}$ is a sequence of points, we say that $y_{k} \rightarrow \infty C_{P}$ if, for all $c \in C_{P}$ there exists $N=N(c)$ so that $k \geq N \Longrightarrow y_{k}-c \in C_{P}$.

4.2. TheOREM C. Suppose $\mathbf{G}$ is a semisimple algebraic group over $\mathbb{Q}$, that $D=$ $G / K$ is a Hermitian symmetric space, $F \subset \bar{D}$ is a rational boundary component, $\mathbf{P} \subset \mathbf{G}$ is the maximal parabolic subgroup which normalizes $F, L_{P}=G_{h} G_{\ell}$ is its Levi quotient, and $C_{P}=G_{\ell} / K_{\ell} \subset \mathfrak{z}$ is the associated self adjoint homogeneous cone. Let $\left\{x_{k}\right\} \subset D$ be a sequence of points. Assume that

(1) $\phi_{\ell}\left(x_{k}\right) \rightarrow \infty C_{P}$,

(2) $\Phi_{\ell}\left(x_{k}\right)$ converges to some point $c \in{\overline{\left[C_{P}\right]}}^{R B S}$ in the reductive Borel-Serre compactification of $\left[C_{P}\right]$, and

(3) $\Phi_{h}\left(x_{k}\right)$ converges to some point $t \in F$.

Then the sequence $\left\{x_{k}\right\}$ converges in $\bar{D}^{R B S}$ to the point $x_{\infty}=\bar{\Psi}_{P}(t, c)$.

4.3 Preliminaries to the proof. Write $x_{k}=u_{k}^{\prime} b_{k}^{\prime} m_{k}^{\prime} K_{P} \in P / K_{P}=D$ relative to the canonical rational Langlands decomposition $P=\mathcal{U}_{P} A_{P}\left(x_{0}\right) M_{P}\left(x_{0}\right)$ of $P$. There is a canonical positive generator $\beta \in \chi\left(A_{P}\right)$ of the (1-dimensional) character module. We would like to say that the sequence $\left\{x_{k}\right\}$ converges in $\bar{D}^{R B S}$ provided

$\left(\mathrm{a}^{\prime}\right) \quad \beta\left(b_{k}^{\prime}\right)=f_{\beta}^{P}\left(x_{k}\right) \rightarrow \infty$ and

$\left(\mathrm{b}^{\prime}\right)$ the sequence $\Phi_{P}\left(x_{k}\right)$ converges in $\bar{D}_{P}^{R B S}$,

since $(1) \Longrightarrow\left(a^{\prime}\right)$ and $(2),(3) \Longrightarrow\left(b^{\prime}\right)$. Unfortunately it is not true that conditions $\left(\mathrm{a}^{\prime}\right)$ and $\left(\mathrm{b}^{\prime}\right)$ guarantee convergence in the reductive Borel-Serre compactification. Instead, we must verify the criteria of Lemma RBS $\S 1.3 .6$. 
4.4. First reduction. The limit point $c$ lies in some RBS boundary component $D_{Q_{\ell}}$ of $\left[C_{P}\right]$ which corresponds to some rational parabolic subgroup, say, $Q_{\ell} \subseteq G_{\ell}$. Then $Q:=\mathcal{U}_{P} i_{x_{0}}\left(Q_{\ell} G_{h}\right)$ is independent of the choice of basepoint, and it is the parabolic subgroup which corresponds to the RBS boundary component $\bar{\Psi}_{P}\left(F \times D_{Q_{\ell}}\right)$ of $D$ which contains the limit point $x_{\infty}$. After conjugating by an element of $\mathbf{G}(\mathbb{Q})$ (if necessary), we may assume that $\mathbf{Q} \supset \mathbf{Q}_{0}$, i.e., that $\mathbf{Q}$ is standard. We may also assume the basepoint $x_{0} \in D$ is rational for $\mathbf{Q}_{0}$. Then $\mathbf{S}=i_{x_{0}} \mathbf{S}\left(\mathbf{Q}_{0}\right)$ is a rationally defined maximal $\mathbb{Q}$-split torus in $\mathbf{G}$ and

$$
\mathbf{S} \subset \mathbf{Q}_{0} \subset \mathbf{Q} \subset \mathbf{P} \subset \mathbf{G}
$$

Let $\Delta=\Delta(S, G)$ denote the resulting set of simple roots. Then the (maximal) parabolic subgroup $\mathbf{P}$ corresponds to the subset $\Delta-\{\beta\}$ (for some $\beta \in \Delta$ ), and the parabolic subgroup $\mathbf{Q}$ corresponds to some subset $I \subset \Delta-\{\beta\}$. By Lemma RBS (§1.3.6) we need to show

(a) $\Phi_{Q}\left(x_{k}\right) \in D_{Q}$ converges to the point $x_{\infty}=\bar{\Psi}_{P}(t, c) \in D_{Q}$,

(b) $f_{\alpha}^{Q_{0}}\left(x_{k}\right) \rightarrow \infty$ for all $\alpha \in \Delta-I$

The mapping $\Phi_{Q}: D \rightarrow D_{Q}$ factors as the composition in the following diagram, which is easily seen (using 1.1 .12 ) to be commutative,

$$
\begin{aligned}
& D \underset{\Phi_{P}}{\longrightarrow} D_{P} \stackrel{\cong}{\stackrel{\cong}{\Psi}} F \times\left[C_{P}\right] \\
& \Phi_{\bar{Q}} \downarrow \quad \downarrow I \times \Phi_{Q_{\ell}} \\
& D_{Q} \stackrel{\cong}{\Psi} F \times D_{Q_{\ell}}
\end{aligned}
$$

(where $\bar{Q}=\nu_{P}(Q)=G_{h} Q_{\ell}$ is the image of $Q$ in the Levi quotient of $P$ ). Hypothesis (2) and Corollary 1.3.8 guarantee that $\Phi_{Q_{\ell}}\left(\Phi_{\ell}\left(x_{k}\right)\right) \rightarrow c \in D_{Q_{\ell}}$. Together with hypothesis (3), this implies that $\Phi_{Q}\left(x_{k}\right) \rightarrow x_{\infty}$, which proves statement (a) above. Moreover, it implies statement $\left(\mathrm{b}^{\prime}\right)$ above, that

$$
\Phi_{P}\left(x_{k}\right) \rightarrow x_{\infty} \text { in } \bar{D}_{P}^{R B S}
$$

which we shall need in the next paragraph.

Now let us verify condition (b). By (4.4.2) and Lemma RBS (§1.3.6) we have

$$
f_{\alpha}^{\bar{Q}_{0}}\left(\Phi_{P}\left(x_{k}\right)\right) \rightarrow \infty \text { for all } \alpha \in \Delta-(I \cup\{\beta\})
$$

where $\bar{Q}_{0}=\nu_{P}\left(Q_{0}\right) \subset L_{P}$. Now apply (1.3.3) (with $x$ replaced by $x_{k}, I$ replaced by $\Delta-\{\beta\}$, and $Q$ replaced by $\left.Q_{0}\right)$. This gives

$$
f_{\alpha}^{Q_{0}}\left(x_{k}\right) \rightarrow \infty \text { for all } \alpha \in \Delta-(I \cup\{\beta\})
$$


Thus, in order to prove Theorem C ( $\S 4.2)$, it remains to show that the hypotheses imply:

$$
f_{\beta}^{Q_{0}}\left(x_{k}\right) \rightarrow \infty
$$

The splitting $L_{P}=G_{h} G_{\ell}$ induces a splitting $\nu_{P}\left(Q_{0}\right)=Q_{0 h} Q_{0 \ell}$ such that $Q_{0 h}$ is a minimal rational parabolic subgroup of $G_{h}$ and $Q_{0 \ell}$ is a minimal rational parabolic subgroup of $G_{\ell}$, each of which has an associated canonical rational Langlands decomposition:

$$
Q_{0 h}=\mathcal{U}_{0 h} A_{0 h}\left(z_{0}\right) M_{0 h}\left(z_{0}\right) \quad Q_{0 \ell}=\mathcal{U}_{0 \ell} A_{0 \ell}(e) M_{0 \ell}(e)
$$

Hence the canonical rational Langlands decomposition of $Q_{0}$ is given by

$$
Q_{0}=\mathcal{U}_{P} i_{x_{0}}\left(\mathcal{U}_{0 h} \mathcal{U}_{0 \ell}\right) i_{x_{0}}\left(A_{0 h}\left(z_{0}\right) A_{0 \ell}(e)\right) i_{x_{0}}\left(M_{0 h}\left(z_{0}\right) M_{0 \ell}(e)\right)
$$

so $x_{k} \in D=Q_{0} / K_{Q_{0}}$ may be expressed as follows:

$$
x_{k}=u_{P}^{(k)} u_{0 h}^{(k)} u_{0 \ell}^{(k)} a_{0 h}^{(k)} a_{0 \ell}^{(k)} m_{0 h}^{(k)} m_{0 \ell}^{(k)} K_{Q_{0}}
$$

Then

$$
f_{\beta}^{Q_{0}}\left(x_{k}\right)=\beta\left(a_{0 h}^{(k)} a_{0 \ell}^{(k)}\right)
$$

Since $\Phi_{h}\left(x_{k}\right)=u_{0 h}^{(k)} a_{0 h}^{(k)} m_{0 h}^{(k)} \in G_{h} / K_{h} \cong F$ converges, we see that the sequence $a_{0 h}^{(k)}$ converges to some element $a_{0 h}^{\infty} \in A_{0 h}$. Hence, in order to verify (4.4.3) it suffices to prove that $\beta\left(a_{0 \ell}^{(k)}\right) \rightarrow \infty$.

Set $y_{k}=\Phi_{\ell}\left(x_{k}\right)=u_{0 \ell}^{(k)} a_{0 \ell}^{(k)} m_{0 \ell}^{(k)} K_{0 \ell} \in G_{0 \ell} / K_{0 \ell} \cong C_{P}$. Then $\beta\left(a_{0 \ell}^{(k)}\right)=f_{\beta}^{Q_{0 \ell}}\left(y_{k}\right)$ so it suffices to prove the following:

Proposition 4.5. Suppose $\mathbf{G}$ is a semisimple algebraic group over $\mathbb{Q}$, that $D=G / K$ is a Hermitian symmetric space, $\mathbf{Q}_{0} \subset G$ is a minimal rational parabolic subgroup and $x_{0} \in D$ is a basepoint which is rational for $Q_{0}$. Let $S=S_{Q_{0}}\left(x_{0}\right) \subset Q_{0}$ be the resulting maximal $\mathbb{Q}$-split torus. Let $\Delta=\Delta(S, G)$ denote the simple rational roots of $G$ occurring in $\mathcal{U}_{Q_{0}}$. Fix $\beta \in \Delta$ and let $\mathbf{P}=\mathbf{P}(\Delta-\{\beta\}) \subset \mathbf{G}$ be the maximal rational proper parabolic subgroup corresponding to the subset $\Delta-\{\beta\}$, with Levi quotient $\nu_{P}: P \rightarrow L_{P}=G_{h} G_{\ell}$ and corresponding self adjoint homogeneous cone $C_{P}=G_{\ell} / K_{\ell} \subset \mathfrak{z}$ with induced basepoint $e \in C_{P}$. Let $Q_{0 \ell}=\nu_{P}\left(Q_{0}\right) \cap G_{\ell}$ be the corresponding minimal rational parabolic subgroup of $G_{\ell}$ and let $f_{\beta}^{Q_{0 \ell}}: C_{P} \rightarrow$ $(0, \infty)$ be the resulting root function on $C_{P}$. Let $\left\{y_{k}\right\} \in C_{P}$ be a sequence of points and suppose that $y_{k} \rightarrow \infty C_{P}$. Then $f_{\beta}^{Q_{0 \ell}}\left(y_{k}\right) \rightarrow \infty$. 
We do not know any simple proof of this fact, although it is easy to verify in special cases (e.g., $G=S p(2 n, \mathbb{R}), S U(n, 1)$, or the Hilbert modular cases). The general proof requires explicit formulae for the roots of $\mathbf{G}$. In $\S 6.7$ we will prove the analog of Proposition 4.5 for algebraic groups $\mathbf{G}$ which are simple over $\mathbb{R}$, and in $\S 6.8$ we will prove proposition 4.5 for algebraic groups $\mathbf{G}$ which are simple over $\mathbb{Q}$. The general case follows from this. (The converse of Proposition 4.5 is false: the condition that $y_{k} \rightarrow \infty C_{P}$ also implies that $f_{\theta}^{Q_{0 \ell}}\left(y_{k}\right) \rightarrow \infty$ for a certain additional characters $\theta$ of $A_{\ell}$.)

5. Real roots of $\mathbf{G}$. Throughout this section we suppose that $\mathbf{G}$ is a semisimple algebraic group defined over $\mathbb{R}$ (and for much of the chapter, $\mathbf{G}$ is assumed to be simple over $\mathbb{R}$ ). Most of the chapter consists of the explicit description of the roots of $\mathbf{G}$ and their relationship to the associated Jordan algebras; these facts are recalled from $[\mathrm{BB}],[\mathrm{AMRT}]$, and $[\mathrm{He}]$. We use this description to prove a special case of Proposition 4.5 at the end of the chapter. Suppose $A$ is a torus in $G$ and $\mathfrak{a}=\operatorname{Lie}(A)$ is its Lie algebra. So as to agree with the notation in [BS], in this $\S 5$ only, we consider each root $\phi \in \Phi(\mathfrak{a}, \mathfrak{g})$ to be a linear functional on $\mathfrak{a}$, and we denote the corresponding character of $A$ by $a \mapsto a^{\phi}$.

5.1. Let $\mathbf{G}$ be a semisimple algebraic group defined over $\mathbb{R}$. Let $G=\mathbf{G}(\mathbb{R})^{0}$ be the connected component of the group of real points, and let $K \subset G$ be a maximal compact subgroup. This corresponds to a choice of basepoint $x_{0} \in D=$ $G / K$ and a Cartan involution on $G$. Let $\mathfrak{g}=\mathfrak{g}_{\mathbb{R}}=\operatorname{Lie}(G)$ denote the Lie algebra of $G$, with Cartan decomposition $\mathfrak{g}=\mathfrak{k} \oplus \mathfrak{p}$. We suppose the symmetric space $D=G / K$ is Hermitian. So there is an invariant complex structure $J: \mathfrak{p} \rightarrow \mathfrak{p}$ with $J^{2}=-1$. Extending $J$ to a complex linear involution on the complexification $\mathfrak{p}_{\mathbb{C}}$ determines a decomposition $\mathfrak{p}_{\mathbb{C}}=\mathfrak{p}_{+} \oplus \mathfrak{p}_{-}$into $\pm i$ eigenspaces of $J$; hence $\mathfrak{g}_{\mathbb{C}}=\mathfrak{k}_{\mathbb{C}} \oplus \mathfrak{p}_{+} \oplus \mathfrak{p}_{-}$. Let $\mathfrak{t} \subset \mathfrak{k}$ be a (compact) Cartan subgroup and $\Phi_{\mathbb{C}}=\Phi\left(\mathfrak{t}_{\mathbb{C}}, \mathfrak{g}_{\mathbb{C}}\right)$ be the roots of $\mathfrak{t}_{\mathbb{C}}$ in $\mathfrak{g}_{\mathbb{C}}$. Then we have the root decomposition

$$
\mathfrak{g}_{\mathbb{C}}=\mathfrak{t}_{\mathbb{C}} \oplus \sum_{\phi \in \Phi_{\mathbb{C}}} \mathfrak{g}_{\mathbb{C}}^{\phi}
$$

As in [BB], [He] $\S \mathrm{VI}(3.1)$ and $\S \mathrm{VIII}$ (7.1), it is possible to choose root vectors $e_{\phi} \in \mathfrak{g}_{\mathbb{C}}^{\phi}$ and vectors $h_{\phi} \in i$ such that

(1) $\left[e_{\phi}, e_{-\phi}\right]=h_{\phi}$.

(2) $\bar{e}_{\phi}=e_{-\phi}$ whenever $e_{\phi} \in \mathfrak{p}_{ \pm}$.

(3) $\psi\left(h_{\phi}\right)=\frac{2\langle\psi, \phi\rangle}{\langle\phi, \phi\rangle}$ for all $\psi \in \Phi_{\mathbb{C}}$.

Set $x_{\phi}=e_{\phi}+e_{-\phi}$, and $y_{\phi}=i\left(e_{\phi}-e_{-\phi}\right)$ and let $\pi_{+}=\left\{\phi \in \Phi_{\mathbb{C}} \mid e_{\phi} \in \mathfrak{p}_{+}\right\}$. Then $\left\{x_{\phi}, y_{\phi}\right\}_{\phi \in \pi_{+}}$form a basis of $\mathfrak{p}$. For any $\phi \in \Phi_{\mathbb{C}}$ set $\epsilon_{\phi}=\frac{1}{2}\left(y_{\phi}-i h_{\phi}\right)$. The proof of the following is a direct computation: 
LEMMA 5.1.1. For all $\phi \in \Phi_{\mathbb{C}}$ we have $\left[\frac{1}{2} x_{\phi}, \epsilon_{\phi}\right]=\epsilon_{\phi}$.

5.2. In this section we recall the explicit description of the roots of $G$ relative to a real split torus, under the additional assumption that $G$ is simple over $\mathbb{R}$. As in [He] $\oint$ VIII (7.4), choose a maximal set of strongly orthogonal roots $\left\{\gamma_{1}^{\prime}, \gamma_{2}^{\prime}, \ldots, \gamma_{r}^{\prime}\right\} \subset \Phi_{\mathbb{C}}$ such that $\mathfrak{a}=\sum_{i=1}^{r} \mathbb{R} x_{i}$ is a maximal abelian subgroup of $\mathfrak{p}$. (Here, and in what follows, we use the notation $x_{i}=x_{\gamma_{i}^{\prime}}, y_{i}=y_{\gamma_{i}^{\prime}}$, etc.) Let $\Phi_{\mathbb{R}}=\Phi(\mathfrak{a}, \mathfrak{g})$ denote the roots of $\mathfrak{a}$ in $\mathfrak{g}=\mathfrak{g}_{\mathbb{R}}$, so $\mathfrak{g}=Z(\mathfrak{a})+\sum_{\phi \in \Phi_{\mathbb{R}}} \mathfrak{g}^{\phi}$. (Here, $Z(\mathfrak{a})$ denotes the centralizer of $\mathfrak{a}$ in $\mathfrak{g}$.) Define $\gamma_{1}, \gamma_{2}, \ldots, \gamma_{r} \in \mathfrak{a}^{*}$ by

$$
\gamma_{i}\left(x_{j}\right)=2 \delta_{i j}(\text { for } 1 \leq i, j \leq r)
$$

Then each $\gamma_{i} \in \Phi_{\mathbb{R}}$ is a root of multiplicity one ([BB] $\S 1.15$, [AMRT] p. 185) and there is an explicit description of $\Phi_{\mathbb{R}}$ in terms of these elements. If $D=G / K$ is irreducible then there are two possibilities. The roots are the nonzero elements in the collection

$$
\begin{array}{ll}
\Phi_{\mathbb{R}}=\left\{\frac{ \pm \gamma_{i} \pm \gamma_{j}}{2}\right\}_{1 \leq i \leq j \leq r} \quad \text { case } \mathrm{C}_{r} \\
\Phi_{\mathbb{R}}=\left\{\frac{ \pm \gamma_{i} \pm \gamma_{j}}{2}, \frac{ \pm \gamma_{i}}{2}\right\}_{1 \leq i \leq j \leq r} & \text { case } \mathrm{BC}_{r} .
\end{array}
$$

Choose a linear order on the set of roots $\Phi_{\mathbb{R}}$. We assume that the ordering $\left\{\gamma_{1}, \gamma_{2}, \ldots, \gamma_{r}\right\}$ is chosen so that $i<j$ iff $\gamma_{i}>\gamma_{j}$. Then the resulting set of simple roots

$$
\Delta_{\mathbb{R}}=\left\{\alpha_{1}, \alpha_{2}, \ldots, \alpha_{r}\right\}
$$

is given as follows:

$$
\begin{array}{lll}
\alpha_{i}=\frac{1}{2}\left(\gamma_{i}-\gamma_{i+1}\right) & & \text { for } 1 \leq i \leq r-1 \\
\alpha_{r}=\gamma_{r} & & \text { case } \mathrm{C}_{r} \\
\alpha_{r}=\frac{1}{2} \gamma_{r} & & \text { case } \mathrm{BC}_{r},
\end{array}
$$

and the positive roots $\Phi_{\mathbb{R}}^{+}$are given by

$$
\begin{array}{ll}
\Phi_{\mathbb{R}}^{+}=\left\{\frac{\gamma_{i}+\gamma_{j}}{2}\right\}_{1 \leq i \leq j \leq r} \cup\left\{\frac{\gamma_{i}-\gamma_{j}}{2}\right\}_{1 \leq i<j \leq r} & \text { case } \mathrm{C}_{r} \\
\Phi_{\mathbb{R}}^{+}=\left\{\frac{\gamma_{i}+\gamma_{j}}{2}\right\}_{1 \leq i \leq j \leq r} \cup\left\{\frac{\gamma_{i}-\gamma_{j}}{2}\right\}_{1 \leq i<j \leq r} \cup\left\{\frac{\gamma_{i}}{2}\right\}_{1 \leq i \leq r} & \text { case } \mathrm{BC}_{r} .
\end{array}
$$

5.3. Parabolic subgroups. Let $\mathbf{Q}_{0}$ be the minimal (real) parabolic subgroup of $\mathbf{G}$ whose group of real points $Q_{0}=\mathbf{Q}_{0}(\mathbb{R})$ is generated by $N=\exp (\mathfrak{n})$ and by $Z(A)$ where $A=\exp (\mathfrak{a})$ and $\mathfrak{n}=\sum\left\{\mathfrak{g}^{\phi} \mid \phi \in \Phi_{\mathbb{R}}^{+}\right\}$. The parabolic subgroups which contain $\mathbf{Q}_{0}$ are called standard. Let $\beta \in \Delta_{\mathbb{R}}$, say $\beta=\alpha_{n}$ in (5.2.2). Let 
$\mathbf{P}=\mathbf{P}\left(\Delta-\left\{\alpha_{n}\right\}\right)$ denote the corresponding maximal proper standard parabolic subgroup. In this section we will explicitly describe the Lie algebra of $P$.

Set $w_{n}=x_{1}+x_{2}+\cdots+x_{n}$. Define

$$
\mathfrak{u}=\sum\left\{\mathfrak{g}^{\phi} \mid \phi \in \Phi_{\mathbb{R}}^{+} \text {and } \phi\left(w_{n}\right)>0\right\} .
$$

The sum in (5.3.1) is over

$$
\begin{array}{ll}
\left\{\frac{\gamma_{i}+\gamma_{j}}{2}\right\}_{1 \leq i \leq j \leq n} \cup\left\{\frac{\gamma_{i} \pm \gamma_{j}}{2}\right\}_{\substack{1 \leq i \leq n \\
j>n}} & \text { case } C_{r} \\
\left\{\frac{\gamma_{i}+\gamma_{j}}{2}\right\}_{1 \leq i \leq j \leq n} \cup\left\{\frac{\gamma_{i} \pm \gamma_{j}}{2}\right\}_{\substack{1 \leq i \leq n \\
j>n}} \cup\left\{\frac{\gamma_{i}}{2}\right\}_{1 \leq i \leq n} & \text { case } B C_{r} .
\end{array}
$$

(The possible values $\phi\left(w_{n}\right)$ are $0, \pm 1, \pm 2$ so the 1-parameter subgroup generated by $w_{n}$ acts on $\mathfrak{u}$ with eigenvalues 0,1 , and 2 .) Set

$$
\mathcal{U}_{P}=\exp (\mathfrak{u}) \text { and } A_{P}=\bigcap_{j \neq n} \operatorname{ker}\left(\alpha_{j}\right) .
$$

Then

$$
P=P\left(\Delta_{\mathbb{R}}-\left\{\alpha_{n}\right\}\right)=Z\left(A_{P}\right) \mathcal{U}_{P}
$$

has unipotent radical $\mathcal{U}_{P}$ and Levi factor $L_{P}=Z\left(A_{P}\right)=G_{h} G_{\ell}$ for which the simple (real) roots may be illustrated in the following Dynkin diagrams:

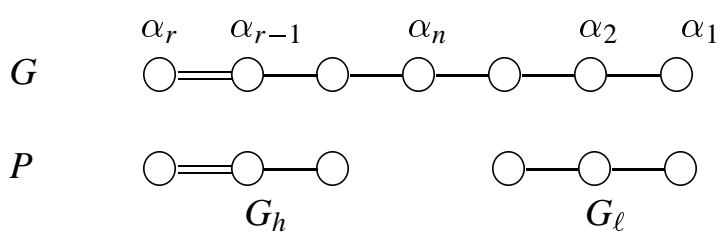

Let $\mathfrak{m}(\mathfrak{a})=Z(\mathfrak{a}) \cap \mathfrak{k}$ be the intersection of $\mathfrak{k}$ with the centralizer of $\mathfrak{a}$. This "compact factor" appears in the minimal parabolic subgroup $Q_{0}$ and hence in each standard parabolic subgroup. Write $A_{\ell}=\exp \left(\mathfrak{a}_{\ell}\right)$ and $A_{h}=\exp \left(\mathfrak{a}_{h}\right)$ where

$$
\mathfrak{a}_{\ell}=\sum_{i=1}^{n} \mathbb{R} x_{i}=\bigcap_{i=n+1}^{r} \operatorname{ker}\left(\gamma_{i}\right)=\bigcap_{i=n+1}^{r} \operatorname{ker}\left(\alpha_{i}\right)
$$

and

$$
\mathfrak{a}_{h}=\sum_{i=n+1}^{r} \mathbb{R} x_{i}=\bigcap_{i=1}^{n} \operatorname{ker}\left(\gamma_{i}\right)
$$


(It is not true that $\mathfrak{a}_{h}=\bigcap_{1 \leq i \leq n} \operatorname{ker}\left(\alpha_{i}\right)$ but (5.2.2) implies $\mathfrak{a}_{h} \subset \bigcap_{1 \leq i \leq n-1} \operatorname{ker}\left(\alpha_{i}\right)$.) Then

$$
\begin{gathered}
\operatorname{Lie}\left(G_{\ell}\right)=\mathfrak{a}_{\ell}+\sum_{\substack{\phi= \pm\left(\gamma_{i}-\gamma_{j}\right) / 2 \\
1 \leq i<j \leq n}}\left(\mathfrak{g}^{\phi}+\left[\mathfrak{g}^{\phi}, \mathfrak{g}^{-\phi}\right] \cap \mathfrak{m}(\mathfrak{a})\right) \\
\operatorname{Lie}\left(G_{h}\right)=\mathfrak{a}_{h}+\sum_{\phi}\left(\mathfrak{g}^{\phi}+\left[\mathfrak{g}^{\phi}, \mathfrak{g}^{-\phi}\right] \cap \mathfrak{m}(\mathfrak{a})\right),
\end{gathered}
$$

where the second sum is taken over all nonzero $\phi$ in the collection

$$
\begin{array}{ll}
\phi \in\left\{\frac{ \pm \gamma_{i} \pm \gamma_{j}}{2}\right\}_{n<i<j \leq r} & \text { in case } \mathrm{C}_{r} \\
\phi \in\left\{\frac{ \pm \gamma_{i} \pm \gamma_{j}}{2}\right\}_{n<i<j \leq r} \cup\left\{\frac{ \pm \gamma_{j}}{2}\right\}_{n<j} & \text { in case } \mathrm{BC}_{r} .
\end{array}
$$

5.4. Jordan algebra. Let

$$
\mathfrak{z}=\sum\left\{\mathfrak{g}^{\phi} \mid \phi=\frac{\gamma_{i}+\gamma_{j}}{2}, \text { for } 1 \leq i \leq j \leq n\right\}
$$

denote the center of $\mathfrak{u}$. The parabolic group $P$ acts on $\mathfrak{z}$ via the adjoint action. The subgroup $G_{\ell}$ acts with an open orbit $C=C_{P}=G_{\ell} \cdot e$ which is an open self adjoint homogeneous cone in $\mathfrak{z}$ with respect to the positive definite inner product $\langle x, y\rangle=-B(x, \sigma(y))$, where $B$ denotes the Killing form and $\sigma$ denotes the Cartan involution. Then $K_{\ell}=K \cap G_{\ell}$ is the stabilizer of the basepoint $e \in C_{P}$ and we obtain a diffeomorphism

$$
G_{\ell} / K_{\ell} \cong C_{P}
$$

If we denote by $\mathfrak{g}_{\ell}=\mathfrak{k}_{\ell} \oplus \mathfrak{p}_{\ell}=\left(\mathfrak{k} \cap \mathfrak{g}_{\ell}\right) \oplus\left(\mathfrak{p} \cap \mathfrak{g}_{\ell}\right)$ the Cartan decomposition of $\mathfrak{g}_{\ell}=\operatorname{Lie}\left(G_{\ell}\right)$, then the differential of (5.4.1) gives an isomorphism

$$
\mathfrak{p}_{\ell} \cong T_{1}\left(G_{\ell} / K_{\ell}\right) \cong T_{e} C_{P} \cong \mathfrak{z}
$$

which is given by $x \mapsto \phi(x)(e)$ where

$$
\phi: \mathfrak{g}_{\ell} \rightarrow \operatorname{End}(\mathfrak{z})
$$

is the differential of the adjoint action $G_{\ell} \rightarrow G L(\mathfrak{z})$. The vectorspace $\mathfrak{z}$ has the following Jordan algebra structure: for any $a \in \mathfrak{z}$ there is (by 5.4.2) a unique element $T_{a} \in \mathfrak{p}_{\ell}$ such that $\phi\left(T_{a}\right)(e)=a$. Then $a \bullet b=\phi\left(T_{a}\right)(b)$ for $a, b \in \mathfrak{z}$. It follows that the closure $\bar{C}_{P}=\left\{x^{2} \mid x \in \mathfrak{z}\right\}$. 
For each $j(1 \leq j \leq n)$ set $\epsilon_{j}=\frac{1}{2}\left(y_{j}-i h_{j}\right)$. It follows from Lemma 5.1.1 that

$$
\epsilon_{j} \in \mathfrak{g}^{\gamma_{j}} \text { and }\left[\frac{1}{2} x_{j}, \epsilon_{j}\right]=\epsilon_{j}
$$

Proposition 5.5. The collection $\left\{\epsilon_{1}, \epsilon_{2}, \ldots, \epsilon_{n}\right\}$ is a complete set of mutually orthogonal idempotents of $\mathfrak{z}$, and $e=\sum_{j=1}^{n} \epsilon_{j}$ is the identity element, relative to this Jordan algebra structure.

Proof 5.6. By (5.4.4), $T_{\epsilon_{j}}=\frac{1}{2} x_{j}$ because $\left[\frac{1}{2} x_{j}, \epsilon\right]=\left[\frac{1}{2} x_{j}, \epsilon_{j}\right]=\epsilon_{j}$ so $\epsilon_{j} \bullet \epsilon_{j}=\epsilon_{j}$ and $\epsilon_{i} \bullet \epsilon_{j}=0$ (for $i \neq j$ ). The collection is complete since this is a maximal set of idempotents and $\sum_{j=1}^{n} \mathbb{R} x_{j}$ is a maximal abelian subalgebra in $\mathfrak{p}_{\ell} \cong \mathfrak{z}$. The identification of $e$ with the basepoint is in [AMRT] p. 242.

5.7. Root function. The minimal parabolic subgroup $\mathbf{Q}_{0} \subset \mathbf{P}$ determines a minimal parabolic subgroup $Q_{0 \ell}=\nu_{P}\left(Q_{0}\right) \cap G_{\ell} \subset G_{\ell}$ (where $\nu_{P}: P \rightarrow P / \mathcal{U}_{P}=$ $L_{P}$ denotes the projection to the Levi quotient). Associated to the simple root $\beta=\alpha_{n} \in \Delta_{\mathbb{R}}$ (cf. (5.2.2) and $\S 1.2$ ) we have the root function

$$
f=f_{\alpha_{n}}^{Q_{0 \ell}}: C_{P} \rightarrow(0, \infty)
$$

If $g \in Q_{0 \ell}$ is given by $g=u a m \in \mathcal{U}_{Q_{0 \ell}} A_{Q_{0 \ell}} M_{Q_{0 \ell}}$ relative to the canonical real Langlands decomposition of $Q_{0 \ell}$, then $f(g e)=a^{\alpha_{n}}$. It follows from (5.3.3) that $\gamma_{n+1}$ is trivial on $A_{\ell}$ so by (5.2.2) we have,

$$
f(g e)=\left\{\begin{aligned}
a^{\gamma_{n} / 2} & \text { if } n<r \\
a^{\gamma_{n}} & \text { if } n=r \text { in case } \mathrm{C}_{r} \\
a^{\gamma_{n} / 2} & \text { if } n=r \text { in case } \mathrm{BC}_{r} .
\end{aligned}\right.
$$

Proposition 5.8. Suppose $\mathbf{G}$ is $\mathbb{R}$-simple. Let $\left\{y_{k}\right\} \subset C_{P}$ be a sequence of points in $C_{P}$ and suppose that $y_{k} \rightarrow \infty C_{P}$. Then $f\left(y_{k}\right) \rightarrow \infty$.

Proof 5.9. Since $\epsilon_{n}$ is an idempotent, we have $\epsilon_{n} \in \partial C_{P}$. Therefore $\left\langle y_{k}, \epsilon_{n}\right\rangle \rightarrow$ $\infty$. Write $y_{k}=g_{k} e$ with $g_{k} \in Q_{0 \ell}$. Then $\left\langle g_{k} e, \epsilon_{n}\right\rangle=\left\langle e, g_{k}^{\tau} \epsilon_{n}\right\rangle \rightarrow \infty$ where $g^{\tau}$ denotes the transpose of $g$. Now let $f_{1}=\epsilon_{1}, f_{2}=\epsilon_{1}+\epsilon_{2}, \ldots, f_{n}=\epsilon_{1}+\cdots+\epsilon_{n}=e$. The parabolic subgroup $Q_{0 \ell}$ is the subgroup of $G$ which normalizes the flag

$$
0 \subset V_{1}\left(f_{1}\right) \subset V_{1}\left(f_{2}\right) \subset \cdots \subset V_{1}\left(f_{n}\right)=\mathfrak{z}
$$

In fact, for each $m$ with $1 \leq m \leq n$ the maximal parabolic subgroup $P_{m}$ (which corresponds to the simple root $\alpha_{m}$ ) preserves the subspace

$$
\mathfrak{z} m=\sum_{1 \leq i, j \leq m} \mathfrak{g}^{\left(\gamma_{i}+\gamma_{j}\right) / 2}
$$


since it is the Lie algebra of the center of the unipotent radical of $P_{m}$. Hence $P_{1} \cap P_{2} \cap \cdots \cap P_{n}$ preserves the flag $0 \subset \mathfrak{z}_{1} \subset \mathfrak{z}_{2} \subset \cdots \subset \mathfrak{z}_{n}$. So $Q_{0 \ell}^{\tau}$ is the subgroup of $G$ which normalizes the flag

$$
\mathfrak{z} \supset V_{0}\left(f_{1}\right) \supset V_{0}\left(f_{2}\right) \supset \cdots \supset V_{0}\left(f_{n}\right)=\{0\} .
$$

But $V_{0}\left(f_{n-1}\right)=V_{1}\left(\epsilon_{n}\right)=\mathfrak{g}^{\gamma_{n}}$ is one dimensional ([BB] $\S 1.15$, [AMRT] p. 185). Let $g^{\tau} \in Q_{0 \ell}^{\tau}$. Then $g^{\tau} \cdot \epsilon_{n}=\lambda \epsilon_{n}$ for some $\lambda=\lambda\left(g^{\tau}\right) \in \mathbb{R}$. Then $\lambda$ is a real character of $Q_{0 \ell}^{\tau}$, it is trivial on $\mathcal{U}_{Q_{0 \ell}^{\tau}}$ and on $M_{Q_{0 \ell}^{\tau}}$ and it coincides with $\gamma_{n}$ on $A_{Q_{0 \ell}^{\tau}}=A_{Q_{0 \ell}}$ (because $\mathfrak{g}^{\gamma_{n}}$ is the subspace of $\mathfrak{g}$ on which $A_{Q_{0 \ell}}$ acts with weight $\gamma_{n}$ ). Hence $\left\langle e, g_{k}^{\tau} \epsilon_{n}\right\rangle=\gamma_{n}\left(a_{k}\right)\left\langle e, \epsilon_{n}\right\rangle=a_{k}^{\gamma_{n}} .1 \rightarrow \infty$ where $g_{k}=u_{k} a_{k} m_{k} \in \mathcal{U}_{Q_{0 \ell}} A_{Q_{0 \ell}} M_{Q_{0 \ell}}$. It follows that $f\left(y_{k}\right)=a_{k}^{\gamma_{n}} \rightarrow \infty$.

\section{Rational theory.}

6.1. In this section we complete the proof of Proposition 4.5. Throughout we assume that $\mathbf{G}$ is an algebraic group defined over $\mathbb{Q}$ which is semisimple over $\mathbb{Q}$, and that $D=G / K$ is a Hermitian symmetric space. We refer to the statement of Proposition 4.5 for the definitions and choices of the following items, which will be fixed throughout this chapter: $\mathbf{Q}_{0} \subset \mathbf{G}, x_{0} \in D, \mathbf{S}=\mathbf{S}_{\mathbf{Q}_{0}}\left(x_{0}\right) \subset \mathbf{Q}_{0}$, $\Delta_{\mathbb{Q}}=\Delta(S, G), \beta \in \Delta_{\mathbb{Q}}, \mathbf{P}=\mathbf{P}\left(\Delta_{\mathbb{Q}}-\{\beta\}\right), \nu_{P}: P \rightarrow L_{P}=G_{h} G_{\ell}, C_{P}=G_{\ell} / K_{\ell} \subset$ $\mathfrak{z} \subset \mathfrak{u}=\operatorname{Lie}\left(\mathcal{U}_{P}\right), e \in C_{P}, Q_{0 \ell}=\nu_{P}\left(Q_{0}\right) \cap G_{\ell}$, and $f=f_{\beta}^{Q_{0 \ell}}: C_{P} \rightarrow(0, \infty)$. Let $\left\{y_{k}\right\} \subset C_{P}$ and suppose that $y_{k} \rightarrow \infty C_{P}$. We must show that $f\left(y_{k}\right) \rightarrow \infty$.

Throughout $\S 6.2$ to $\S 6.7$ we assume that $\mathbf{G}$ is simple over $\mathbb{R}$.

6.2. We must compare the real roots and the rational roots. Let $\mathbf{T}$ be a maximal $\mathbb{R}$ split torus in $\mathbf{G}$ with $\mathbf{S} \subset \mathbf{T}$. We assume that $\mathbf{T}$ is defined over $\mathbb{Q}$. Choose a minimal real parabolic subgroup $\mathbf{P}_{0} \subset \mathbf{G}$ such that $\mathbf{S} \subset \mathbf{T} \subset \mathbf{P}_{0} \subset \mathbf{Q}_{0}$. Let us denote the corresponding groups of real points by

$$
A_{Q_{0}}=\mathbf{S}(\mathbb{R})^{0} \subset A_{P_{0}}=\mathbf{T}(\mathbb{R})^{0} \subset \mathbf{P}_{0} \subset \mathbf{Q}_{0}
$$

Associated to these choices there are root systems $\Phi_{\mathbb{Q}}=\Phi(S, G)$ and $\Phi_{\mathbb{R}}=$ $\Phi(T, G)$, positive roots $\Phi_{\mathbb{Q}}^{+}, \Phi_{\mathbb{R}}^{+}$(which occur in the unipotent radical of $P_{0}$ and $Q_{0}$ respectively), and simple roots $\Delta_{\mathbb{Q}} \subset \Phi_{\mathbb{Q}}^{+}, \Delta_{\mathbb{R}} \subset \Phi_{\mathbb{R}}^{+}$. A fundamental result of Baily and Borel [BB] states:

LEMMA 6.3. For each $\phi \in \Delta_{Q}$ there exists a unique simple root $\phi^{\prime} \in \Delta_{\mathbb{R}}$ such that $\phi=\phi^{\prime} \mid \mathbf{S}$. Let $\Delta_{\mathbb{R}}^{\prime} \subset \Delta_{\mathbb{R}}$ denote the resulting subset of $\Delta_{\mathbb{R}}$. If $\phi \in \Delta_{\mathbb{R}}$ then

$$
\phi \notin \Delta_{\mathbb{R}}^{\prime} \text { iff } \phi \mid \mathbf{S}=1
$$

(i.e. the remaining simple roots are trivial on $\mathbf{S}$ ). 
6.4. The root $\beta \in \Delta_{\mathbb{Q}}$ corresponds (by Lemma 6.3) to a unique real root $\beta^{\prime} \in \Delta_{\mathbb{R}}$. It follows from (6.3.1) that $\beta^{\prime}$ gives rise to the same maximal parabolic subgroup,

$$
\mathbf{P}=\mathbf{P}\left(\Delta_{\mathbb{Q}}-\{\beta\}\right)=\mathbf{P}\left(\Delta_{\mathbb{R}}-\left\{\beta^{\prime}\right\}\right)
$$

because

$$
\mathbf{S}\left(\Delta_{\mathbb{R}}-\left\{\beta^{\prime}\right\}\right)=\bigcap_{\phi \in \Delta_{\mathbb{R}}-\left\{\beta^{\prime}\right\}} \operatorname{ker}(\phi) \subset \mathbf{T}(\mathbb{R})
$$

coincides with

$$
\mathbf{S}\left(\Delta_{\mathbb{Q}}-\{\beta\}\right)=\bigcap_{\phi \in \Delta_{\mathbb{Q}}-\{\beta\}} \operatorname{ker}(\phi) \subset \mathbf{S}(\mathbb{R}) .
$$

We obtain a minimal real parabolic subgroup $P_{0 \ell}=\nu_{P}\left(P_{0}\right) \cap G_{\ell} \subset G_{\ell}$ and an associated root function $f_{\beta^{\prime}}^{P_{0 \ell}}: C_{P} \rightarrow(0, \infty)$.

LeMma 6.5. The root functions $f_{\beta^{\prime}}^{P_{0 \ell}}$ and $f_{\beta}^{Q_{0 \ell}}$ coincide.

Proof 6.6. Define

$$
\mathbf{T}_{\text {an }}=\bigcap_{\phi \in \chi(\mathbf{T})_{\mathbb{Q}}} \operatorname{ker}(\phi) \subset \mathbf{T}
$$

to be the maximal $\mathbb{Q}$-anisotropic torus in $\mathbf{T}$, where $\chi(\mathbf{T})_{\mathbb{Q}}$ denotes the group of rationally defined characters of $\mathbf{T}$. Then $A_{a n}=\mathbf{T}_{\text {an }}(\mathbb{R})^{0} \subset \mathbf{M}_{\mathbf{Q}_{0}}$. Since $\mathbf{S} \subset \mathbf{T}$ is a maximal $\mathbb{Q}$-split torus in $\mathbf{T}$, we have an almost direct product (cf. [B] $\S 8.14$ ), $\mathbf{T}=\mathbf{S} . \mathbf{T}_{\text {an }}$ from which it follows that

$$
A_{P_{0}}=A_{Q_{0}} A_{a n} .
$$

In fact, this is a direct product since the intersection is finite but each factor is torsion free. If $a \in A_{P_{0}}$ decomposes as $a=b b_{a n}$ in (6.6.1), then $b$ is the unique element in $A_{Q_{0}}$ such that

$$
\phi^{\prime}(a)=\phi^{\prime}(b)=\phi(b) \text { for all } \phi \in \Delta_{\mathbb{Q}},
$$

since the corresponding elements $\phi^{\prime} \in \Delta_{\mathbb{R}}^{\prime}$ form a rational basis of the module $\chi(\mathbf{T})_{\mathbb{Q}} \otimes \mathbb{Q}$ of rationally defined characters of $\mathbf{T}$.

Now let $y=g e$ for some $g \in P_{0 \ell} \subset Q_{0 \ell}$. Decompose

$$
g=u a_{\mathbb{R}} m_{\mathbb{R}}=u a_{\mathbb{Q}} m_{\mathbb{Q}}
$$


relative to the canonical real Langlands decomposition $P_{0 \ell}=\mathcal{U}\left(P_{0 \ell}\right) A\left(P_{0 \ell}\right) M\left(P_{0 \ell}\right)$ and relative to the canonical rational Langlands decomposition $Q_{0 \ell}$ $=\mathcal{U}\left(Q_{0 \ell}\right) A\left(Q_{0 \ell}\right) M\left(Q_{0 \ell}\right)$. Using (6.6.1), write $a_{\mathbb{R}}=b b_{a n}$ with $b \in A_{Q_{0}}$ and $b_{a n} \in A_{a n}$. We claim that $b \in A_{Q_{0 \ell}}$ and that $b_{a n} \in M\left(Q_{0 \ell}\right)$. It suffices to show that $b \in G_{\ell}$ (from which it follows that $b_{a n}=a_{\mathbb{R}} b^{-1} \in G_{\ell}$ as well). By (5.3.3) the linear part $A_{Q_{0 \ell}}$ is the intersection of the kernels of certain real simple roots $\left\{\alpha_{1}, \alpha_{2}, \ldots, \alpha_{n}\right\} \subset \Delta_{\mathbb{R}}$. If $\alpha_{i} \notin \Delta_{\mathbb{R}}^{\prime}$ then $\alpha_{i}(b)=1$ by (6.3.1). If $\alpha_{i} \in \Delta^{\prime}$ then by (6.6.2), $\alpha_{i}(b)=\alpha_{i}\left(a_{\mathbb{R}}\right)=1$, which completes the proof of the claim.

Now it follows that $g=u \cdot b \cdot\left(b_{a n} m\right)$ is the rational Langlands decomposition of $g$, in other words, that $b=a_{\mathbb{Q}}$. We conclude (again using (6.6.2)) that

$$
f_{\beta^{\prime}}^{P_{0 \ell}}(y)=\beta^{\prime}\left(a_{\mathbb{R}}\right)=\beta^{\prime}(b) \beta^{\prime}\left(b_{a n}\right)=\beta\left(a_{\mathbb{Q}}\right) \cdot 1=f_{\beta}^{Q_{0 \ell}}(y)
$$

which completes the proof of the lemma.

6.7. Proof of Proposition 4.5 when G is $\mathbb{R}$-simple. Let $\left\{y_{k}\right\} \subset C_{P}$ be a sequence of points in the cone $C_{P}$ with $y_{k} \rightarrow \infty C_{P}$. Then by Proposition 5.8, we have $f_{\beta^{\prime}}^{P_{0 \ell}}\left(y_{k}\right) \rightarrow \infty$, but by Lemma $6.5, f_{\beta^{\prime}}^{P_{0 \ell}}\left(y_{k}\right)=f_{\beta}^{Q_{0 \ell}}\left(y_{k}\right)$.

6.8. The $\mathbb{Q}$-simple case. Throughout $\S 6.8$ to $\S 6.9$ we assume that $\mathbf{G}$ is simple over $\mathbb{Q}$ but is not necessarily simple over $\mathbb{R}$. By Baily-Borel $[\mathrm{BB}]$ and Borel-Tits [BT], there exists an algebraic group $\mathbf{G}^{\prime}$ defined over a totally real number field $k$ such that $\mathbf{G}^{\prime}$ is absolutely simple (and hence is simple over $\mathbb{R}$ ) and

$$
\operatorname{Res}_{k / \mathbb{Q}} \mathbf{G}^{\prime}=\mathbf{G}
$$

(where Res denotes Weil's restrictions of scalars). Let $\sigma_{1}, \ldots, \sigma_{d}$ denote the $d$ different embeddings of $k$ into $\mathbb{R}$ and write $k_{i}=\sigma_{i}(k)=k^{\sigma_{i}}$. Set $\mathbf{G}_{i}=\left(\mathbf{G}^{\prime}\right)^{\sigma_{i}}$. Then there is an isomorphism of real algebraic groups,

$$
\mathbf{G} \cong \mathbf{G}_{1} \times \cdots \times \mathbf{G}_{d}
$$

We have chosen a maximal $\mathbb{Q}$-split torus $\mathbf{S} \subset \mathbf{G}$. There is an isomorphism over $k$ of tori, $\mathbf{S} \cong \mathbf{S}^{\prime}$, where $\mathbf{S}^{\prime} \subset \mathbf{G}^{\prime}$ is a maximal $k$-split torus (and so $\mathbf{S}$ is isomorphic to a maximal $\mathbb{Q}$ split torus in $\operatorname{Res}_{k / \mathbb{Q}}\left(\mathbf{S}^{\prime}\right)$ ). Let $S_{i}=\left(S_{i}\right)^{\sigma_{i}}$ be the corresponding maximal $k_{i}$-split torus in $\mathbf{G}_{i}$. These tori are all isomorphic over $\mathbb{R}$ so we may identify the root systems

$$
\Phi(\mathbf{S}, \mathbf{G}) \leftrightarrow \Phi\left(\mathbf{S}^{\prime}, \mathbf{G}^{\prime}\right) \leftrightarrow \Phi\left(\mathbf{S}_{i}, \mathbf{G}_{i}\right)
$$

and corresponding subsets of positive and simple roots $\Delta_{\mathbb{Q}}=\Delta(\mathbf{S}, \mathbf{G}), \Delta\left(\mathbf{S}^{\prime}, \mathbf{G}^{\prime}\right)$, $\Delta\left(\mathbf{S}_{i}, \mathbf{G}_{i}\right)$ respectively. The chosen simple root $\beta \in \Delta(\mathbf{S}, \mathbf{G})$ corresponds to simple $\operatorname{roots} \beta_{i} \in \Delta\left(\mathbf{S}_{i}, \mathbf{G}_{i}\right)$. 
The minimal and maximal rational parabolic subgroups $\mathbf{Q}_{0} \subset \mathbf{P}=\mathbf{P}\left(\Delta_{\mathbb{Q}}-\right.$ $\{\beta\}) \subset \mathbf{G}$ correspond to minimal and maximal $k_{i}$-parabolic subgroups $\mathbf{Q}_{0 i} \subset$ $\mathbf{P}_{i} \subset \mathbf{G}_{i}$. Then, as real parabolic subgroups, we have an isomorphism

$$
P \cong P_{1} \times \cdots \times P_{d}
$$

Write $C=C_{P}=G_{\ell} / K_{\ell}$ for the self adjoint homogeneous cone associated to $P$, and write $C_{i}=C_{P_{i}}=G_{\ell, i} / K_{\ell, i}$ for the corresponding cones associated to $P_{i}$. Then we also have a diffeomorphism,

$$
C \cong C_{1} \times C_{2} \times \cdots \times C_{d}
$$

Each minimal parabolic subgroup $\mathbf{Q}_{0 i} \subset \mathbf{G}_{i}$ determines a corresponding minimal parabolic subgroup $\mathbf{Q}_{0 \ell, i} \subset \mathbf{G}_{\ell, i}$ with root function

$$
f_{i}=f_{\beta_{i}}^{Q_{0 \ell, i}}: C_{i} \rightarrow(0, \infty)
$$

6.9. Proof of Proposition 4.5 when $\mathbf{G}$ is $\mathbb{Q}$-simple. If $y_{k} \in C$ is a sequence of points, with $y_{k} \rightarrow \infty C$, then the corresponding factors $y_{k}^{(i)} \in C_{i}$ also satisfy $y_{k}^{(i)} \rightarrow \infty C_{i}$. Since $G_{i}$ is $\mathbb{R}$-simple, we may apply (6.7) to each factor (replacing $\mathbb{Q}$ by $k_{i}$ in the statement and proof of Case 1) to conclude that each of the root functions $f_{i}\left(y_{k}^{(i)}\right) \rightarrow \infty$.

On the other hand, we claim that $f_{\beta}\left(y_{k}\right)=f_{i}\left(y_{k}^{(i)}\right)$ for each $i$, from which the result follows. Write $y_{k}=g_{k} e$ with $g_{k} \in Q_{0}$. Then $g_{k}=\left(g_{k}^{(1)}, \ldots, g_{k}^{(d)}\right) \in$ $Q_{0,1} \times \cdots \times Q_{0, d}$, each of which may be decomposed according to compatibly chosen Langlands decompositions,

$$
g_{k}^{(i)}=u_{k}^{(i)} a_{k}^{(i)} m_{k}^{(i)}
$$

However, by our identification (6.8.1) of the roots and the torus we have,

$$
f_{\beta}\left(y_{k}\right)=\beta\left(a_{k}\right)=\beta_{i}\left(a_{k}^{(i)}\right) \text { for each } i
$$

This completes the proof of Proposition 4.5.

\section{Statement and proof of Theorem A.}

7.1. Let $\mathbf{G}$ be a semisimple algebraic group defined over $\mathbb{Q}$. Let $G=\mathbf{G}(\mathbb{R})$ be the group of real points. Let us assume that $G=\operatorname{Aut}^{0}(D)$ is the connected component of the group of automorphisms of a Hermitian symmetric space $D$. Let $\Gamma \subset \mathbf{G}(\mathbb{Q})$ be a neat arithmetic subgroup. Set $X=\Gamma \backslash D$. Let $\Sigma=\left\{\Sigma_{F}\right\}$ be a $\Gamma$-admissible collection of polyhedral cone decompositions of the homogeneous cones $C_{F}$ (as $F$ runs over all the rational boundary components of $D$ ). In [AMRT] 
this data is used to construct a toroidal compactification $\bar{X}_{\Sigma}^{\text {tor }}$ of $X$. Let us say that the collection of polyhedral decompositions $\Sigma$ is $\Gamma$-sufficiently fine if, for every rational parabolic subgroup $\mathbf{P}=\mathbf{P}(F)$ the polyhedral decomposition $\Sigma_{F}$ of $C_{F}^{*}$ is $\Gamma_{\ell}$-sufficiently fine (where $\Gamma_{\ell}=G_{\ell} \cap \nu_{P}(\Gamma \cap P)$, with $\nu_{P}: P \rightarrow L_{P}=G_{h} G_{\ell}$ the projection to the Levi quotient).

Let $\bar{X}^{R B S}$ denote the reductive Borel-Serre compactification and let $\bar{X}^{B B}$ denote the Baily-Borel Satake compactification of $X$. Let

$$
X \stackrel{d}{\longrightarrow} X \times X \hookrightarrow \bar{X}^{R B S} \times \bar{X}_{\Sigma}^{\text {tor }}
$$

denote the diagonal inclusion of $X$ into the product of two compactifications of $X$, and let $\widehat{X}$ denote the closure of the image of $X$ in $\bar{X}^{R B S} \times \bar{X}_{\Sigma}^{\text {tor }}$. Let $\bar{a} \in \bar{X}_{\Sigma}^{\text {tor }}$, and define

$$
\widehat{X}(\bar{a})=\left\{\bar{b} \in \bar{X}^{R B S} \mid(\bar{b}, \bar{a}) \in \widehat{X}\right\} \subset \bar{X}^{R B S} .
$$

Then $\widehat{X}(\bar{a})=\theta_{1} \theta_{2}^{-1}(\bar{a}) \cong \theta_{2}^{-1}(\bar{a})$ where $\theta_{1}, \theta_{2}$ denote the projections from $\widehat{X}$ to $\bar{X}^{R B S}$ and $\bar{X}_{\Sigma}^{\text {tor }}$ respectively.

THEOREM A 7.2. Suppose the collection $\left\{\Sigma_{F}\right\}$ of polyhedral cone decompositions is $\Gamma$-sufficiently fine. Then for any $\bar{a} \in \bar{X}_{\Sigma}^{\text {tor }}$ the set $\widehat{X}(\bar{a})$ is contractible.

In fact we will show that the set $\widehat{X}(\bar{a})$ is canonically homeomorphic to a set of the form $[\sigma]^{R B S}$ which, by Theorem $\mathrm{B}$, is contractible.

COROLlary 7.3. If the collection $\left\{\Sigma_{F}\right\}$ of polyhedral decompositions is $\Gamma$ sufficiently fine, then the resolution $g: \bar{X}_{\Sigma}^{\text {tor }} \rightarrow \bar{X}^{B B}$ is homotopic to a mapping $g^{\prime}: \bar{X}_{\Sigma}^{t o r} \rightarrow \bar{X}^{B B}$ which factors through the projection $\mu: \bar{X}^{R B S} \rightarrow \bar{X}^{B B}$.

Moreover it is possible to choose $g^{\prime}$ so that $g(\bar{S})=g^{\prime}(\bar{S})$ for each stratum $S$ of $\bar{X}^{t o r}$. The mapping $g^{\prime}$ may also be taken to be the identity on the complement $K$ of a regular neighborhood of the boundary $\partial \bar{X}_{\Sigma}^{\text {tor }}=\bar{X}_{\Sigma}^{\text {tor }}-X$. (Such a closed subset $K \subset X$ is described in [Le] and [Sa] where it is shown to be homotopy equivalent to $X$.)

7.4. Proof of corollary. Choose a triangulation of $\bar{X}^{t o r}$ which is a compatible with the stratification [G]. In Proposition 8.2 we construct a homotopy inverse $\tau: \bar{X}_{\Sigma}^{t o r} \rightarrow \hat{X}$ to the projection $\theta_{2}$. Take $g^{\prime}$ to be the composition

$$
\bar{X}_{\Sigma}^{\text {tor }} \stackrel{\tau}{\longrightarrow} \hat{X} \stackrel{\theta_{1}}{\longrightarrow} \bar{X}^{R B S} \stackrel{\mu}{\longrightarrow} \bar{X}^{B B}
$$

Since $\tau g(\sigma) \subset \sigma$ for each (closed) simplex $\sigma \subset \bar{X}^{\text {tor }}$ we see that $g^{\prime}(\bar{S})=\mu \theta_{1} \tau(\bar{S})=$ 
$g \theta_{2} \tau(\bar{S})=g(\bar{S})$ for each stratum $S$ of $\bar{X}^{t o r}$. By Addendum 8.3 the mapping $\tau$ may be taken to be the identity on $K \subset X$.

7.5. The remainder of $\S 7$ is devoted to the proof of Theorem A. Let us recall some of the notation involved in the construction of the toroidal resolution. If we suppose that $\bar{a} \in \partial \bar{X}_{\Sigma}^{\text {tor }}$ then its image in the Baily-Borel compactification $\bar{X}^{B B}$ lies in some stratum which we may take to be an arithmetic quotient of some rational boundary component $F$. Let $\mathbf{P}$ be the associated maximal rational parabolic subgroup of $\mathbf{G}$, so $P=\mathbf{P}(\mathbb{R})=\operatorname{Norm}(F)$ normalizes $F$. Let $\mathcal{U}_{\mathbf{F}}=\mathcal{U}_{\mathbf{P}}$ denote its unipotent radical and $\mathbf{Z}_{\mathbf{F}}=\mathbf{Z}_{\mathbf{P}}$ denote the center of $\mathcal{U}_{\mathbf{P}}$. Set $\Lambda_{F}=\mathbf{Z}_{\mathbf{F}}(\mathbb{R}) \cap \Gamma$. The exponential mapping $\mathfrak{z}_{F} \rightarrow \mathbf{Z}_{\mathbf{F}}(\mathbb{R})$ determines a vectorspace structure on the group $\mathbf{Z}_{\mathbf{F}}(\mathbb{R})$ which in turn admits an integral structure so that the inclusion $\Lambda_{F} \hookrightarrow \mathbf{Z}_{\mathbf{F}}(\mathbb{R})$ induces a vectorspace isomorphism $\Lambda_{F} \otimes \mathbb{R} \cong \mathbf{Z}_{\mathbf{F}}(\mathbb{R})$ which takes $\Lambda_{F}$ isomorphically to $\mathbf{Z}_{\mathbf{F}}(\mathbb{Z})$. Then $T(F)=\mathbf{Z}_{\mathbf{F}}(\mathbb{C}) / \Lambda_{\mathbf{F}}$ is an algebraic torus with cocharacter group $\Lambda_{F} \cong \chi_{*}(T(F))$ : each $\lambda \in \Lambda_{F}$ determines a mapping $\mathbb{C} \rightarrow \mathbf{Z}_{\mathbf{F}}(\mathbb{C})$ by $t \mapsto \lambda t$ and this determines a cocharacter $\mathbb{C}^{*}=\mathbb{C} / \mathbb{Z} \rightarrow \mathbf{Z}_{\mathbf{F}}(\mathbb{C}) / \Lambda_{F}$. In summary we will use these isomorphisms to make the canonical identifications

$$
\chi_{*}(T(F)) \otimes \mathbb{R} \cong \mathbf{Z}_{\mathbf{F}}(\mathbb{R}) \cong \mathfrak{z}_{F} .
$$

The Levi quotient $L_{P}=P / \mathcal{U}_{P}$ decomposes as an almost direct product, $L_{P}=$ $G_{\ell} \times G_{h}$ of a "linear" and a Hermitian factor. The action by conjugation of $G_{\ell}$ on $Z_{F}$ induces a diffeomorphism between $G_{\ell} / K_{\ell}$ and the open orbit $C_{F} \subset Z_{F}$. The adjoint action of $G_{\ell}$ on $\mathfrak{z}=\operatorname{Lie}\left(Z_{F}\right)$ induces a diffeomorphism between $G_{\ell} / K_{\ell}$ and the open orbit $C_{P} \subset \mathfrak{z}$. The exponential mapping exp: $C_{P} \rightarrow C_{F}$ is compatible with these diffeomorphisms. Abusing the notation of (4.1.5), we will write $\phi_{\ell}: D \rightarrow C_{F}$ and $\Phi_{\ell}: D \rightarrow\left[C_{F}\right]$ for the resulting projections. (And, as in (4.1.5) we also have a projection $\Phi_{h}: D \rightarrow F$.) Set $D(F)=\mathbf{Z}_{\mathbf{F}}(\mathbb{C}) \cdot D$ (the product taken in the compact dual symmetric space). Then there is a holomorphic isomorphism given by Siegel coordinates, [AMRT] p. 235, [S] $\S \mathrm{III}$ (Thm. 7.1), $[\mathrm{KW}]$

$$
D(F) \cong F \times\left(\mathcal{U}_{F} / Z_{F}\right) \times \mathbf{Z}_{\mathbf{F}}(\mathbb{C})
$$

with the following properties:

(1) There exists a real bilinear form $h_{t}$ which depends real analytically on the parameter $t \in F$ such that

$$
D=\left\{(t, w, z) \mid \operatorname{Im}(z)-h_{t}(w, w) \in C_{F}\right\} .
$$

(2) For all $(t, w, z) \in D$ we have $\Phi_{h}(t, w, z)=t$ and

$$
y=\phi_{\ell}(t, w, z)=\operatorname{Im}(z)-h_{t}(w, w) .
$$


(Abusing notation, we will write $\left(\mathcal{U}_{F} / Z_{F}\right)=\mathbb{C}^{k}$ for simplicity.) Then the torus $T(F)$ acts freely on the quotient $\Lambda_{F} \backslash D(F)$ with quotient $D(F)^{\prime}=F \times \mathbb{C}^{k}$. So $\Lambda(F) \backslash D(F)$ is a "torus bundle" over $D(F)^{\prime}$, the fibers of which will be compactified in the following paragraph.

The choice $\Sigma_{F}$ of polyhedral cone decomposition determines a partial compactification $(T(F))_{\Sigma_{F}}$ of the torus $T(F)$. This gives rise to a partial compactification

$$
\left(\Lambda_{F} \backslash D(F)\right)_{\Sigma_{F}}=\Lambda_{F} \backslash D(F) \times_{T(F)}(T(F))_{\Sigma_{F}}
$$

Let $\left(\Lambda_{F} \backslash D\right)_{\Sigma_{F}}$ denote the interior of the closure of $\Lambda_{F} \backslash D(F)$ in $\left(\Lambda_{F} \backslash D(F)\right)_{\Sigma_{F}}$. The action of $\Lambda_{F} \backslash \Gamma_{F}$ on $\Lambda_{F} \backslash D(F)$ extends to an action of $\Lambda_{F} \backslash \Gamma_{F}$ on the partial compactification $\left(\Lambda_{F} \backslash D(F)\right)_{\Sigma_{F}}$. (Here, $\Gamma_{F}=\Gamma \cap P$.)

7.5.4. Fact. ([AMRT] p. 250) There exists a collection of maps $\pi_{F}$ : $\left(\Lambda_{F} \backslash D\right)_{\Sigma_{F}} \rightarrow \bar{X}_{\Sigma}^{\text {tor }}$ such that:

(1) Each $\pi_{F}$ is open and analytic with discrete fibers.

(2) The mapping $\pi_{F}$ commutes with the action of $\Lambda_{F} \backslash \Gamma_{F}$ and, near the boundary it induces an embedding $\left(\Lambda_{F} \backslash D\right)_{\Sigma_{F}} /\left(\Lambda_{F} \backslash \Gamma_{F}\right) \hookrightarrow \bar{X}_{\Sigma}^{t o r}$.

(3) The union, taken over all $\Gamma$ conjugacy classes of rational boundary components $F$,

$$
\coprod_{F}\left(\Lambda_{F} \backslash D\right)_{\Sigma_{F}} \rightarrow \bar{X}_{\Sigma}^{t o r}
$$

is surjective.

7.6. Notation. In order to avoid double subscripts, whenever no confusion will arise, we will write $\mathbf{Z}$ for $\mathbf{Z}_{\mathbf{F}}, \Lambda$ for $\Lambda_{F}$, and $\Sigma$ for $\Sigma_{F}$. Let $\mathbb{T}=Z(\mathbb{R}) / \Lambda$ be the compact torus in $T(F)=\mathbf{Z}(\mathbb{C}) / \Lambda$. The map ord: $\mathbf{Z}(\mathbb{C}) \rightarrow \mathbf{Z}(\mathbb{R})$ given by $\operatorname{ord}(x+i y)=y$ induces a map $T(F) \rightarrow \mathbf{Z}(\mathbb{R})$. From $[$ AMRT] I $\S 1.1$ the map ord extends to a map

$$
\text { ord: } T(F)_{\Sigma} \rightarrow Z_{\Sigma}
$$

where

$$
Z_{\Sigma}=\mathbf{Z}(\mathbb{R}) \cup \coprod_{\sigma \in \Sigma} \mathcal{O}_{\sigma}
$$

with $\mathcal{O}_{\sigma}=\mathbf{Z}(\mathbb{R}) / \mathbf{L}_{\sigma}$. Each point in $\mathcal{O}_{\sigma}$ can be expressed as $\mathrm{V}+L_{\sigma}$ with $\mathrm{V} \in L_{\sigma}^{\perp}$. Following [AMRT] we use the (confusing) notation $\mathrm{V}+\infty \sigma$ to denote such a point. This notation may be justified by considering the topology on $Z_{\Sigma}$. If $\left\{y_{n}\right\} \subset \mathbf{Z}(\mathbb{R})$ is a sequence, then $y_{n} \rightarrow \mathrm{V}+\infty \sigma$ provided the following holds: write $y_{n}=y_{n}^{\prime}+y_{n}^{\prime \prime}$ 
with $y_{n}^{\prime} \in L_{\sigma}^{\perp}$ and $y_{n}^{\prime \prime} \in L_{\sigma}$. Then $y_{n}^{\prime} \rightarrow \mathrm{V}$ and for any $w \in L_{\sigma}$, if $n$ is sufficiently large then $y_{n}^{\prime \prime}-w \in \sigma$ (cf. Definition 2.7.2).

The map ord induces a homeomorphism $\mathbb{T} \backslash T(F)_{\Sigma} \cong Z_{\Sigma}$. Hence we may express every element in $T(F)_{\Sigma}-T(F)$ as $x^{\prime}+i(y+\infty \sigma)$ where $x^{\prime} \in \mathbb{T}$. However $x^{\prime}$ is only uniquely determined modulo the sub compact torus $\mathbb{T}_{\sigma}=\mathcal{O}_{\sigma} / \Lambda_{\sigma}$ where $\Lambda_{\sigma}=\Lambda /\left(\Lambda \cap L_{\sigma}\right)$.

Using Siegel coordinates (7.5.1), if $a=(t, w, z) \in D(F)$ then we will write $a^{\prime}=\left(t, w, z^{\prime}\right)$ for its image in $\Lambda_{F} \backslash D(F) \cong F \times\left(\mathcal{U}_{F} / Z_{F}\right) \times T(F)$. The projections $\Phi_{h}$ and $\phi_{\ell}$ pass to this quotient with $\Phi_{h}\left(a^{\prime}\right)=t$ and $\phi_{\ell}\left(a^{\prime}\right)=\operatorname{Im}\left(z^{\prime}\right)-h_{t}(w, w) \in C_{F} \subset$ $\mathbf{Z}(\mathbb{R})$. Now suppose $a^{\prime} \in\left(\Lambda_{F} \backslash D\right)_{\Sigma}$ is a point on the boundary corresponding to some (closed) polyhedral cone $\sigma \in \Sigma_{F}$ with $\sigma^{o} \subset C_{F}$. We will write

$$
a^{\prime}=\left(t, w, x^{\prime}+i \mathrm{~V}+i \infty \sigma\right)
$$

if there is a sequence $a_{n}^{\prime}=\left(t_{n}, w_{n}, z_{n}^{\prime}\right) \in \Lambda_{F} \backslash D$ such that

(1) $t_{n} \rightarrow t \in F$,

(2) $w_{n} \rightarrow w \in\left(\mathcal{U}_{F} / Z_{F}\right) \cong \mathbb{C}^{k}$,

(3) $\operatorname{Re}\left(z_{n}^{\prime}\right) \rightarrow x^{\prime} \in \Lambda_{F} \backslash \mathbf{Z}(\mathbb{R})$,

(4) $\phi_{\ell}\left(a_{n}^{\prime}\right) \rightarrow \mathrm{V}+\infty \sigma$,

where $x^{\prime} \in \Lambda_{F} \backslash Z_{F}(\mathbb{R})$ and $\mathrm{v} \in L_{\sigma}^{\perp}$, the perpendicular complement to the linear space $L_{\sigma}$ spanned by $\sigma$.

7.7. Lift to the partial compactification. The purpose of this section is to lift the subset $\widehat{X}(\bar{a}) \subset \bar{X}^{R B S}$ to the reductive Borel-Serre partial compactification $\bar{D}^{R B S}$. Suppose, as above, that $\bar{a} \in \partial \bar{X}_{\Sigma}^{t o r}$ projects to some stratum $X_{F} \subset \bar{X}^{B B}$ which is an arithmetic quotient of some rational boundary component $F$ with normalizing maximal parabolic subgroup $P$. Fix any point $a^{\prime} \in\left(\Lambda_{F} \backslash D\right)_{\Sigma}$ so that $\pi_{F}\left(a^{\prime}\right)=\bar{a}$. Let us say that a point $b \in \bar{D}^{R B S}$ is closure related to $a^{\prime} \in\left(\Lambda_{F} \backslash D\right)_{\Sigma}$ if there exists a sequence $a_{n} \in D$ with the following two properties:

(1) $\quad a_{n}^{\prime} \rightarrow a^{\prime}$ in $\left(\Lambda_{F} \backslash D\right)_{\Sigma}$ and

(2) $a_{n} \rightarrow b$ in $\bar{D}^{R B S}$.

(Here, $a_{n}^{\prime} \in \Lambda_{F} \backslash D$ denotes the image of $a_{n} \bmod \Lambda_{F}$.)

Then $b \in \bar{D}_{P}^{R B S}$ is in the closure of the reductive Borel-Serre boundary component $D_{P}$. Let $\bar{a}_{n} \in \Gamma \backslash D=X$ denote the image of $a_{n}$ modulo $\Gamma$ (so $a_{n} \in D$, $a_{n}^{\prime} \in \Lambda_{F} \backslash D$, and $\left.\bar{a}_{n} \in \Gamma \backslash D\right)$. Then we also have:

(3) $\quad \bar{a}_{n} \rightarrow \bar{a}=\pi_{F}\left(a^{\prime}\right)$ in $\bar{X}_{\Sigma}^{t o r}$ and

(4) $\bar{a}_{n} \rightarrow \bar{b}$ in $\bar{X}^{R B S}=\Gamma \backslash \bar{D}^{R B S}$ 
since the following diagram commutes,

$$
\begin{aligned}
& a_{n} \in D \hookrightarrow \bar{D}^{R B S} \times\left(\Lambda_{F} \backslash D\right)_{\Sigma} \ni\left(b, a^{\prime}\right) \\
& \pi \downarrow \quad \downarrow \pi \times \pi_{F} \\
& \bar{a}_{n} \in X \hookrightarrow \bar{X}^{R B S} \times \bar{X}_{\Sigma}^{\text {tor }} \quad \ni(\bar{b}, \bar{a}) \text {. }
\end{aligned}
$$

Define

$$
\Delta\left(a^{\prime}\right)=\left\{b \in \bar{D}^{R B S} \mid b \text { is closure related to } a^{\prime}\right\} \subset \bar{D}_{P}^{R B S} .
$$

If $\widehat{D}$ denotes the closure of the diagonal embedding of $D$ in $\bar{D}^{R B S} \times\left(\Lambda_{F} \backslash D\right)_{\Sigma}$ and if $\theta_{1}$ and $\theta_{2}$ denote the projections of $\widehat{D}$ to the first and second factors respectively, then $\Delta\left(a^{\prime}\right)=\theta_{1} \theta_{2}^{-1}\left(a^{\prime}\right) \cong \theta_{2}^{-1}\left(a^{\prime}\right)$. The following proposition immediately implies Theorem A.

Proposition 7.8. Let $\bar{a} \in \partial \bar{X}_{\Sigma}^{\text {tor }}$. Choose $F, P$, and $a^{\prime}=\left(t, w, x^{\prime}+i \mathrm{v}+i \infty \sigma\right)$ as in (7.6.1) with $\pi_{F}\left(a^{\prime}\right)=\bar{a}$ and with $\sigma^{o} \subset C_{F}$. Let $\left[C_{F}\right]$ denote the quotient of $C_{F}$ under homotheties, and (4.1.4) let

$$
\bar{\Psi}_{P}: \bar{F}^{R B S} \times{\overline{\left[C_{F}\right]}}^{R B S} \cong \bar{D}_{P}^{R B S}
$$

be the resulting homeomorphism which we will take to be a canonical identification (so we will usually omit explicit mention of $\bar{\Psi}_{P}$ ). Let $[\sigma]^{R B S} \subset{\overline{\left[C_{F}\right]}}^{R B S}$ be the closure of the quotient $\left[\sigma^{o}\right] \subset\left[C_{F}\right]$ in the reductive Borel Serre compactification. Then

(1) the identification $\bar{\Psi}_{P}$ restricts to a homeomorphism

$$
\{t\} \times[\sigma]^{R B S} \stackrel{\cong}{\longrightarrow} \Delta\left(a^{\prime}\right)
$$

(2) If $\Sigma$ is $\Gamma$-sufficiently fine then the mapping $\pi: \bar{D}^{R B S} \rightarrow \bar{X}^{R B S}$ restricts to a homeomorphism

$$
\pi \mid \Delta\left(a^{\prime}\right): \Delta\left(a^{\prime}\right) \stackrel{\cong}{\longrightarrow}(\bar{a}) .
$$

Proof 7.9. It is clear from properties (3) and (4) above that the restriction $\pi \mid \Delta\left(a^{\prime}\right)$ takes $\Delta\left(a^{\prime}\right)$ into $\widehat{X}(\bar{a})$. First let us show that $\pi: \Delta\left(a^{\prime}\right) \rightarrow \widehat{X}(\bar{a})$ is surjective. Fix any $\bar{b} \in \widehat{X}(\bar{a})$. Then $(\bar{b}, \bar{a}) \in \widehat{X}$ so there exists a sequence $\left\{\bar{a}_{n}\right\} \subset X$ so that $\bar{a}_{n} \rightarrow \bar{a}$ in $\bar{X}_{\Sigma}^{t o r}$ and so that $\bar{a}_{n} \rightarrow \bar{b}$ in $\bar{X}^{R B S}$. Since $\pi_{F}$ is a local homeomorphism, there is a unique lift $a_{n}^{\prime} \in\left(\Lambda_{F} \backslash D\right)_{\Sigma}$ of the sequence $\bar{a}_{n}$ so that $a_{n}^{\prime} \rightarrow a^{\prime}$ in 
$\left(\Lambda_{F} \backslash D\right)_{\Sigma}$. As in (7.5.1) write

$$
a_{n}^{\prime}=\left(t_{n}, w_{n}, z_{n}^{\prime}\right)
$$

It follows that $t_{n} \rightarrow t, w_{n} \rightarrow w$ and that $y_{n}:=\phi_{\ell}\left(a_{n}^{\prime}\right) \in C_{F}$.

Claim 7.9.1. There exists a subsequence (which we also denote by $a_{n}^{\prime}$ ) so that the corresponding elements $\left[y_{n}\right] \in\left[C_{F}\right]$ converge to some point $c \in[\sigma]^{R B S}$. (Here, $[y]$ denotes the image of $y$ under the quotient by homotheties, $C_{F} \rightarrow\left[C_{F}\right]$.)

7.9.2. Proof of claim. Since $y_{n} \rightarrow \mathrm{v}+\infty \sigma$, and $\mathrm{v} \in L_{\sigma}^{\perp}$ we may write $y_{n}=y_{n}^{\prime}+y_{n}^{\prime \prime}$ with $y_{n}^{\prime} \in L_{\sigma}^{\perp}$ and $y_{n}^{\prime \prime} \in \sigma^{o}$ for $n$ sufficiently large. Therefore $y_{n}^{\prime} \rightarrow \mathrm{V}$ and $y_{n}^{\prime \prime} \rightarrow \infty \sigma$. Since $\left[y_{n}^{\prime \prime}\right] \in\left[\sigma^{o}\right] \subset[\sigma]^{R B S}$ which is compact, there exists a subsequence (which we denote by by $\left[y_{n}^{\prime \prime}\right]$ as well) which converges, $\left[y_{n}^{\prime \prime}\right] \rightarrow c \in[\sigma]^{R B S}$. By Proposition 2.7.3, the sequence $\left[y_{n}\right]=\left[y_{n}^{\prime}+y_{n}^{\prime \prime}\right]$ converges in ${\overline{\left[C_{F}\right]}}^{R B S}$ to the same point $c$.

7.9.3. Using this claim, and choosing any lift $a_{n} \in D$ of this subsequence we see that

(1) $\phi_{\ell}\left(a_{n}\right)=y_{n} \rightarrow \infty C$,

(2) $\Phi_{h}\left(a_{n}\right)=t_{n} \rightarrow t$,

(3) $\Phi_{\ell}\left(a_{n}\right)=\left[y_{n}\right] \rightarrow c$ in ${\overline{\left[C_{F}\right]}}^{R B S}$.

Here, $\Phi_{h}: D \rightarrow F$ and $\Phi_{\ell}: D \rightarrow\left[C_{F}\right]$ are the canonical projections (4.1.5) (so, if $\psi_{2}: \bar{D}_{P}^{R B S} \rightarrow{\overline{\left[C_{F}\right]}}^{R B S}$ denotes the projection to the second factor, then $\Phi_{\ell}=$ $\left.\psi_{2} \circ \Phi_{P}\right)$. By Theorem C ( $(4.2)$, this implies that the sequence $a_{n} \rightarrow b:=(t, c) \in$ $\bar{D}_{P}^{R B S} \cong \bar{F}^{R B S} \times{\overline{\left[C_{F}\right]}}^{R B S}$. In summary, $b \in \Delta\left(a^{\prime}\right)$ (since $a_{n} \rightarrow b$ and $a_{n}^{\prime} \rightarrow a^{\prime}$ ) and $\pi(b)=\bar{b}$ (by (4) above) which proves that $\pi \mid \Delta\left(a^{\prime}\right)$ is surjective to $\hat{X}(\bar{a})$.

7.9.4. Let us verify part (1) of Proposition 7.8: that $\Delta\left(a^{\prime}\right)=\{t\} \times[\sigma]^{R B S}$ (where $\left.a^{\prime}=\left(t, w, x^{\prime}+i \mathrm{v}+i \infty \sigma\right)\right)$. As in the preceding paragraph, let $\psi_{2}: \bar{D}_{P}^{R B S} \rightarrow$ ${\overline{\left[C_{F}\right]}}^{R B S}$ denote the projection to the second factor. Let $b \in \Delta\left(a^{\prime}\right) \subset \bar{D}_{P}^{R B S}$, say $b=(t, c)$. Choose $\left\{a_{n}\right\} \subset D$ as above, with $a_{n} \rightarrow b$ in $\bar{D}^{R B S}$ and $a_{n}^{\prime} \rightarrow a^{\prime}$ in $\left(\Lambda_{F} \backslash D\right)_{\Sigma}$ as described above. Then (by the same argument as in the proof of the claim above), $\psi_{2}(b)=c \in[\sigma]^{R B S}$. Since $t \in F$ is fixed, we conclude that $\psi_{2} \mid \Delta\left(a^{\prime}\right)$ is injective. To see that it is surjective, let $c \in[\sigma]^{R B S}$ and choose $y_{n} \in \sigma$ so that $\left[y_{n}\right] \rightarrow c$ and define

$$
a_{n}=\left(t, w, x^{\prime}+i \mathrm{~V}+i \lambda_{n} y_{n}+h_{t}(w, w)\right)
$$

where $\lambda_{n} \in \mathbb{R}$ and $\lambda_{n} \rightarrow \infty$. Then $\Phi_{h}\left(a_{n}\right)=t, \Phi_{\ell}\left(a_{n}\right) \rightarrow c$ (again by Proposition 2.7.3), and $\phi_{\ell}\left(a_{n}\right) \rightarrow \infty C_{F}$. So by Theorem C (§4.2), the sequence $a_{n}$ 
converges in $\bar{D}^{R B S}$ to the limit $b=(t, c) \in \Delta\left(a^{\prime}\right)$ which completes the proof of part (1).

7.9.5. Now let us prove that the mapping $\pi: \Delta\left(a^{\prime}\right) \rightarrow \widehat{X}(\bar{a})$ is injective. Since

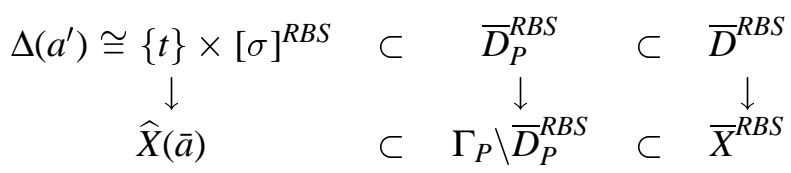

commutes, we see that $\pi$ is injective if and only if the quotient under $\Gamma_{P}$ does not introduce any identifications on $[\sigma]^{R B S}$. Let $\Gamma_{\ell}=\nu_{P}(\Gamma \cap P) \cap G_{\ell}$ where $\nu_{P}: P \rightarrow L_{P}$ is the projection to the Levi quotient. Corresponding to the exact sequence

$$
1 \rightarrow \Gamma_{\ell} \rightarrow \nu_{P}(\Gamma \cap P) \rightarrow \Gamma_{h}^{\prime} \rightarrow 1
$$

there is a fiber bundle $\Gamma_{P} \backslash \bar{D}_{P}^{R B S} \rightarrow \Gamma_{h}^{\prime} \backslash \bar{F}^{R B S}$ with fiber $\Gamma_{\ell} \backslash{\overline{\left[C_{F}\right]}}^{R B S}$. Since $t \in F$ is fixed, it suffices to verify that $\Gamma_{\ell}$ does not introduce any identifications on $[\sigma]^{R B S}$. However this is precisely the assumption that $\sigma$ is $\Gamma_{\ell^{-}}$sufficiently small (§3.1). It is guaranteed by the assumption that $\Sigma$ is $\Gamma$-sufficiently fine ( $\S 7.1)$. This concludes the proof of Proposition 7.8 and hence also of Theorem A.

\section{Contractible cell complexes.}

8.1. In this section we review some standard facts from homotopy theory which are needed for the proof of Theorem B (\$2.8). Throughout this section we suppose that $f: X \rightarrow Y$ is a weakly stratified mapping between two compact (finite dimensional) Whitney stratified spaces. (This means that $f$ takes strata to strata by a smooth submersion.) Fix a triangulation of $Y$.

Proposition 8.2. Suppose that $f$ is surjective and that each fiber $f^{-1}(y)$ is contractible. Then $f$ is a homotopy equivalence. Moreover there exists a homotopy inverse $g: Y \rightarrow X$ such that, for each (closed) simplex $\sigma \subset Y$,

(1) $f g(\sigma) \subset \sigma$ and

(2) the restriction $g \mid \sigma: \sigma \rightarrow f^{-1}(\sigma)$ is a homotopy inverse for the restriction $f^{-1}(\sigma) \rightarrow \sigma$.

In particular, if $Y$ is contractible then $X$ is also contractible.

AdDENDUM 8.3. Suppose that $K \subset Y$ is a closed union of simplices of the chosen triangulation. Suppose that $f^{-1}(K) \rightarrow K$ is a homeomorphism. Then the homotopy inverse $g: Y \rightarrow X$ may be chosen so as to agree with $f^{-1}$ on points of $K$. 
Proof 8.4. This follows from standard results in homotopy theory, however in what follows we will indicate how to construct the homotopy inverse $g$ explicitly. Fix a triangulation of $Y$ which refines the stratification. We will find a mapping $g: Y \rightarrow X$ (which agrees with $f^{-1}$ on $K$ ) and a homotopy $H: X \times I \rightarrow X$ between $H_{0}=I$ and $H_{1}=g f$ so that $H$ is the constant homotopy (from the identity to the identity) on $K \times I$ and so that, for each simplex $\sigma \subset Y$ we have

(1) $f g(\sigma) \subset \sigma$,

(2) $H\left(f^{-1}(\sigma) \times I\right) \subset f^{-1}(\sigma)$.

This is accomplished by induction on the dimension of $\sigma$. For $\operatorname{dim}(\sigma)=0$ let $g(\sigma)$ be any point in the fiber $f^{-1}(\sigma)$ and let $H$ be a homotopy which contracts the fiber $f^{-1}(\sigma)$ to the point $g(\sigma)$. For the inductive step, suppose $\sigma \subset Y$ is an $n$-dimensional simplex with boundary $\partial \sigma$, that $g: \partial \sigma \rightarrow f^{-1}(\partial \sigma)$ has been defined and that a homotopy $H: f^{-1}(\partial \sigma) \times[0,1] \rightarrow f^{-1}(\partial \sigma)$ between $H_{0}=I$ and $H_{1}=g f$ has been constructed. We wish to extend both $g$ and $H$ to all of $\sigma$. If $\sigma \subset K$, these extensions have already been defined: $g=f^{-1}$ and $H$ is the constant homotopy. So we may assume that $\operatorname{int}(\sigma) \subset Y-K$. Since the extensions will be made simplex by simplex without changing choices which were made on previous simplices, we may (for the sake of notational convenience) replace $X$ by $f^{-1}(\sigma)$.

It is possible to triangulate $X$ so that $f^{-1}(\partial \sigma)$ is a union of simplices. In particular, the inclusion $f^{-1}(\partial \sigma) \subset X$ is a cofibration, i.e., it satisfies the homotopy extension property, which we apply to the following situation:

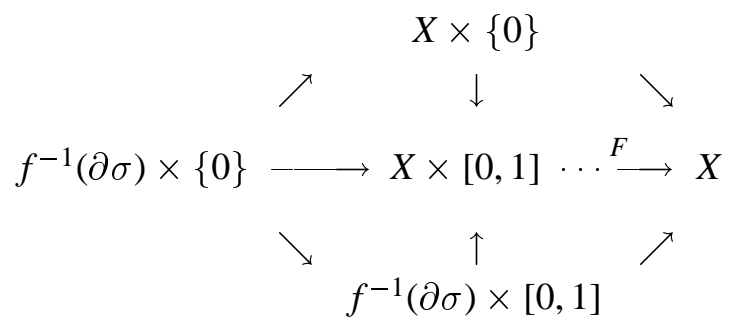

Thus, we obtain a homotopy $F$ between $F_{0}=I$ and some mapping $F_{1}: X \rightarrow X$ which collapses $f^{-1}(\partial \sigma)$ to the section $g(\partial \sigma)$.

Choose a trivialization

$$
f^{-1}\left(\sigma^{o}\right) \cong \sigma^{o} \times A
$$

of the mapping $f$ over the interior $\sigma^{o}$ of the simplex $\sigma$ (such a trivialization exists by Thom's first isotopy lemma) and let $a_{0} \in A$ be a point in the fiber. Let $\phi_{t}: A \rightarrow A$ be a contracting homotopy from $\phi_{0}=I$ to the constant mapping $\phi_{x}(a)=a_{0}$. Define $g^{\prime}: \sigma^{o} \rightarrow f^{-1}\left(\sigma^{o}\right)$ by $g^{\prime}(y)=\left(y, a_{0}\right)$. Then $g^{\prime}$ is a homotopy inverse for $f$ on the interior of $\sigma$, but we must patch together $g^{\prime}$ on the interior with $g$ on the boundary. 
Definition 8.5. The section $g: \sigma \rightarrow X$ is given by

$$
g(y)=\left\{\begin{aligned}
F_{1} g^{\prime}(y) & \text { for } y \in \sigma^{o} \\
g(y) & \text { for } y \in \partial \sigma
\end{aligned}\right.
$$

To verify that $g$ is continuous, choose a strong deformation retraction

$$
\psi: N\left(f^{-1}(\partial \sigma)\right) \rightarrow f^{-1}(\partial \sigma)
$$

from a regular neighborhood of $f^{-1}(\partial \sigma)$ to $f^{-1}(\partial \sigma)$. Suppose $y_{i} \in \sigma^{o}$ is a sequence of points converging to some point $y_{0} \in \partial \sigma$. Then for any choice of metric on $X$ we have

$$
\operatorname{dist}\left(F_{1} g^{\prime}\left(y_{i}\right), g\left(y_{0}\right)\right) \leq \operatorname{dist}\left(F_{1} g^{\prime}\left(y_{i}\right), F_{1} \psi g^{\prime}\left(y_{i}\right)\right)+\operatorname{dist}\left(g f \psi g^{\prime}\left(y_{i}\right), g\left(y_{0}\right)\right)
$$

(since $F_{1}=g f$ on $f^{-1}(\partial \sigma)$ ). The first term goes to 0 because $F_{1}$ is continuous and $\operatorname{dist}\left(g^{\prime}\left(y_{i}\right), \psi g^{\prime}\left(y_{i}\right)\right) \rightarrow 0$ (since $\psi$ is a strong deformation retraction which approaches the identity as the point $g^{\prime}\left(y_{i}\right)$ approaches the boundary $\left.f^{-1}(\partial \sigma)\right)$. To show that the second term goes to 0 , it suffices to show that $f \psi g^{\prime}\left(y_{i}\right) \rightarrow y_{0}$. But

$$
\operatorname{dist}\left(f \psi g^{\prime}\left(y_{i}\right), y_{0}\right) \leq \operatorname{dist}\left(f \psi g^{\prime}\left(y_{i}\right), f g^{\prime}\left(y_{i}\right)\right)+\operatorname{dist}\left(f g^{\prime}\left(y_{i}\right), y_{0}\right)
$$

Again the first term goes to 0 because $\psi \rightarrow I$ on $f^{-1}(\partial \sigma)$ while the second term is $\operatorname{dist}\left(y_{i}, y_{0}\right) \rightarrow 0$.

Now let us define the extension of the homotopy $H$. Choose a collaring of the boundary of the simplex,

$$
r: \sigma \rightarrow[0,1]
$$

so that $r^{-1}(0)=\partial \sigma$ and define the function $T:[0,1] \times[0,1] \rightarrow[0,1]$ by

$$
T(r, t)= \begin{cases}t & \text { if } r+t \geq 1 \\ \frac{r t}{1-t} & \text { if } r+t \leq 1\end{cases}
$$

With respect to the trivialization (8.4.1) define

$$
H_{t}(y, a)=F_{t}\left(y, \phi_{T(r(y), t)}(a)\right) .
$$

Although the function $T$ fails to be continuous at $(r=0, t=1)$ it is easy to see (using the same argument involving $\psi: N\left(f^{-1}(\partial \sigma)\right) \rightarrow f^{-1}(\partial \sigma)$ ) as above) that the function $H_{t}$ is continuous as $y \rightarrow \partial \sigma$. Furthermore for 
$(y, a) \in f^{-1}\left(\sigma^{o}\right)$,

$$
\begin{gathered}
H_{0}(y, a)=F_{0}\left(y, \phi_{0}(a)\right)=(y, a) \\
H_{1}(y, a)=F_{1}\left(y, \phi_{1}(a)\right)=F_{1}\left(g^{\prime}(y)\right)=g f(y, a)
\end{gathered}
$$

as desired. This completes the proof of Proposition 8.2.

\section{Automorphic vectorbundles.}

9.1. Throughout this section, $\mathbf{G}$ denotes a semisimple algebraic group defined over $\mathbb{Q}$, with group of real points $G=\mathbf{G}(\mathbb{R})$. We assume that $G=\operatorname{Aut}^{0}(D)$ is the connected component of the group of automorphisms of a Hermitian symmetric space $D=G / K$, where $K$ is the maximal compact subgroup of $G$ corresponding to a choice of basepoint $x_{0} \in D$. Let $\Gamma \subset \mathbf{G}(\mathbb{Q})$ be a neat arithmetic subgroup. Let $X=\Gamma \backslash D$ and let $\mu: \bar{X}^{R B S} \rightarrow \bar{X}^{B B}$ be its reductive Borel-Serre and Baily-Borel Satake compactifications.

Let $\lambda: K \rightarrow G L(E)$ be a representation of $K$ on some (finite dimensional) complex vectorspace $E$. the homogeneous vectorbundle

$$
\mathbf{E}^{\prime}=G \times_{K} E
$$

is the quotient of $G \times E$ under the equivalence relation $(g, e) \sim\left(g k, \lambda\left(k^{-1}\right) e\right)$ (for all $g \in G, k \in K$, and $e \in E$ ); we will denote the associated equivalence class by $[g, e]$. The group $G$ acts by $g^{\prime}[g, e]=\left[g^{\prime} g, e\right]$ and the quotient under $\Gamma \subset G$ is an automorphic vectorbundle on $X=\Gamma \backslash D$, which we denote by

$$
\mathbf{E}=\Gamma \backslash G \times{ }_{K} E .
$$

Except in special cases, the vectorbundle $\mathbf{E}$ does not admit an extension (even as a topological vectorbundle) to the Baily-Borel compactification.

Proposition 9.2. The automorphic vectorbundle $\mathbf{E}$ has a canonical extension $\overline{\mathbf{E}}^{R B S} \rightarrow \bar{X}^{R B S}$ (as a topological complex vectorbundle) over the reductive BorelSerre compactification.

Proof 9.3. As in $\S 1.1$, let $\mathbf{P} \subset \mathbf{G}$ be a proper rational parabolic subgroup with unipotent radical $\mathcal{U}_{P}$ and Levi quotient $\mathrm{v}_{P}: P \rightarrow L_{P}=A_{P} M_{P}$. Let $\mathrm{v}_{M}: P \rightarrow M_{P}$ denote the further projection to $M_{P}$. Then $K_{P}=K \cap P \subset M_{P}\left(x_{0}\right)$ is a maximal compact subgroup of $M_{P}$ and $\Gamma_{L}=\mathrm{v}_{P}(\Gamma \cap P) \subset M_{P}$ is an arithmetic subgroup of $M_{P}$ and the associated RBS boundary stratum is $X_{P}=\Gamma_{L} \backslash M_{P} / K_{P}$. Restricting the representation $\lambda$ to $K_{P} \subset L_{P}$ we obtain a homogeneous vectorbundle $\mathbf{E}_{\mathbf{P}}^{\prime}=$ 
$M_{P} \times_{K_{P}} E$ on $D_{P}$, which passes to an automorphic vectorbundle

$$
\mathbf{E}_{\mathbf{P}}=\Gamma_{P} \backslash M_{P} \times_{K_{P}} E
$$

on the boundary stratum $X_{P}$. The extension $\overline{\mathbf{E}}^{R B S}$ will be obtained by gluing $\mathbf{E}$ together with these vectorbundles $\mathbf{E}_{\mathbf{P}}$ on the strata.

The projection $\Phi_{P}: P \rightarrow D_{P}$ of (1.3.1) is covered by a mapping of $P$ homogeneous vectorbundles

$$
\Theta_{P}: \mathbf{E}^{\prime}=P \times_{K_{P}} E \rightarrow M_{P} \times_{K_{P}} E=\mathbf{E}_{\mathbf{P}}^{\prime}
$$

which is given by $[g, e] \mapsto\left[\mathrm{v}_{M}(g), e\right]$. It is well defined (since $\lambda(k)=\lambda\left(\mathrm{v}_{M}(k)\right.$ ) for all $k \in K_{P}$ ) and is $P$-equivalent, (where $P$ acts on $M_{P} \times_{K_{P}} E$ by $p \cdot[m, e]=$ $\left.\left[\mathrm{v}_{M}(p) m, e\right]\right)$ and it is an isomorphism on each fiber. It follows that the union $\mathbf{E}^{\prime} \cup \mathbf{E}_{\mathbf{P}}^{\prime}$ admits the unique structure of a $P$-homogeneous vectorbundle on $D \cup D_{P}$ such that any choice of trivialization $\beta: \mathbf{E}_{\mathbf{P}}^{\prime} \rightarrow E$ of $\mathbf{E}_{\mathbf{P}}^{\prime}$ extends continuously to a trivialization of $\mathbf{E}^{\prime} \cup \mathbf{E}_{\mathbf{P}}^{\prime}$ which is given by the composition

$$
\mathbf{E}^{\prime} \underset{\Theta_{P}}{\longrightarrow} \mathbf{E}_{\mathbf{P}}^{\prime} \underset{\beta}{\longrightarrow} \mathbf{E}
$$

If $\mathbf{P} \subset \mathbf{Q}$ then we similarly obtain a $P$-equivariant vectorbundle mapping $\Theta_{P Q}$ : $\mathbf{E}_{\mathbf{Q}}^{\prime} \rightarrow \mathbf{E}_{\mathbf{P}}^{\prime}$ which is compatible with the mappings $\Theta_{P}$ and $\Theta_{Q}$, that is, $\Theta_{P}$ is the composition

$$
\mathbf{E}^{\prime} \underset{\Theta_{Q}}{\longrightarrow} \mathbf{E}_{\mathbf{Q}}^{\prime} \underset{\Theta_{P Q}}{\longrightarrow} \mathbf{E}_{\mathbf{P}}^{\prime}
$$

This endows the union

$$
\mathbf{E}^{\prime} \cup \coprod_{\mathbf{P} \subset \mathbf{G}} \mathbf{E}_{\mathbf{P}},
$$

with the structure of a $\mathbf{G}(\mathbb{Q})$-homogeneous vectorbundle on $\bar{D}^{R B S}$, which then passes to a vectorbundle structure on the quotient by $\Gamma$,

$$
\overline{\mathbf{E}}^{R B S}=\mathbf{E} \cup \coprod_{\{\mathbf{P}\}} \mathbf{E}_{\mathbf{P}},
$$

where the union is taken over a collection of representatives, one from each $\Gamma$-conjugacy class $\{\mathbf{P}\}$ of proper rational parabolic subgroups of $\mathbf{G}$.

9.4. Toroidal compactification. Let $\Sigma=\left\{\Sigma_{F}\right\}$ be a sufficiently fine $\Gamma$ admissible collection of polyhedral cone decompositions of the homogeneous cones $C_{F}$ (as $F$ runs over all the rational boundary components of $D$ ), and let $\bar{X}_{\Sigma}^{\text {tor }}$ 
be the resulting toroidal compactification with its canonical mapping $g: \bar{X}_{\Sigma}^{t o r} \rightarrow$ $\bar{X}^{B B}$. In [M], D. Mumford shows that every homogeneous vectorbundle $\mathbf{E}$ on $X$ admits a canonical extension $\overline{\mathbf{E}}_{\Sigma}^{t o r}$ to the toroidal compactification. In Theorem A and its corollary $(\S 7.2, \S 7.3)$ a mapping

$$
h=\theta_{1} \circ \tau: \bar{X}_{\Sigma}^{t o r} \rightarrow \bar{X}^{R B S}
$$

is constructed, so that $g^{\prime}=\mu \circ h: \bar{X}_{\Sigma}^{t o r} \rightarrow \bar{X}^{B B}$ is homotopic to and is $C^{0}$-close to $g$.

Conjecture 9.5. The topological (complex) vectorbundles $\overline{\mathbf{E}}_{\Sigma}^{\text {tor }}$ and $h^{*}\left(\overline{\mathbf{E}}^{R B S}\right)$ are isomorphic.

School of Mathematics, Institute for Advanced Study, Princeton NJ 08540

DePARTMENT OF MATHEMATics, HAVERford College, HaVerford PA 19041

\section{REFERENCES}

[A] J. Arthur, The $L^{2}$ Lefschetz numbers of Hecke operators, Invent. Math. 97 (1989), 257-290.

[AMRT] A. Ash, D. Mumford, M. Rapoport, and Y. S. Tai, Smooth Compactification of Locally Symmetric Varieties, Math. Sci. Press, Brookline, MA, 1975.

[BB] W. L. Baily, Jr. and A. Borel, Compactification of arithmetic quotients of bounded symmetric domains, Ann. of Math. 84 (1966), 442-528.

[B] A. Borel, Linear Algebraic Groups, 2nd ed. Graduate Texts in Math., vol. 126, Springer-Verlag, New York, 1991.

[BS] A. Borel and J. P. Serre, Corners and arithmetic groups, Comment. Math. Helv. 48 (1973 ), $436-491$.

[BT] A. Borel and J. Tits, Groupes Réductifs, Inst. Hautes Études Sci. Publ. Math. 27 (1965), 55-150.

[FK] J. Faraut and A. Korányi, Analysis on Symmetric Cones, Oxford University Press, Oxford, UK, 1994.

[G] M. Goresky, Triangulation of stratified objects, Proc. Amer. Math. Soc. 72 (1978), 193-200.

[GHM] M. Goresky, G. Harder, and R. MacPherson, Weighted cohomology, Invent. Math. 116 (1994), 139-213.

[GKM] M. Goresky, R. Kottwitz, and R. MacPherson, Discrete series characters and the Lefschetz formula for Hecke operators, Duke Math. J. 89 (1997), 477-554.

[GM1] M. Goresky and R. MacPherson, Local contribution to the Lefschetz fixed point formula, Invent. Math. 111 (1993), 1-33.

[GM2] Lefschetz numbers of Hecke correspondences, The Zeta Function of Picard Modular Surfaces (R. P. Langlands and D. Ramakrishnan, ed.), Centre de Recherches Mathématiques, Univ. de Montréal, Montréal, Canada, 1992, pp. 465-478.

[Ha] R. Hardt, Topological properties of subanalytic sets, Trans. Amer. Math. Soc. 211 (1975), 57-70.

[HC] Harish-Chandra, Representations of semi-simple Lie groups VI, Amer. J. Math. 78 (1956), 564-628.

[HZ] M. Harris and S. Zucker, Boundary cohomology of Shimura varieties II. Hodge theory at the boundary, Invent. Math. 116 (1994), 243-307.

[He] S. Helgason, Differential Geometry, Lie Groups, and Symmetric Spaces, Academic Press, San Diego, CA, 1978

[Hi] H. Hironaka, Subanalytic sets, Number Theory, Algebraic Geometry and Commutative Algebra, Kinokunya, Tokyo, 1973, pp. 453-493. 
[Hu] H. Hudson, Piecewise Linear Topology, W. A. Benjamin, New York, 1969.

[Ji] L. Ji, GCQ of Borel-Serre and toroidal compactifications of locally symmetric spaces, Geom. Funct. Anal. 8 (1998), 978-1015.

[KW] A. Korányi and J. Wolf, Generalized Cayley transformations of bounded symmetric domains, Amer. J. Math. 87 (1965), 899-939.

[Le] E. Leuzinger, An exhaustion of locally symmetric spaces by compact submanifolds with corners, Invent. Math. 121 (1995), 389-410.

[L] E. Looijenga, $L^{2}$ cohomology of locally symmetric varieties, Compositio Math. 67 (1988), 3-20.

[Mc] M. McConnell, Cell decompositions of Satake compactifications for $\operatorname{SL}(n, \mathbb{R})$, preprint, Oklahoma State University, 1994.

[M] D. Mumford, Hirzebruch's proportionality theorem in the non-compact case, Invent. Math. 42 (1977), 239-272,

[MF] D. Mumford and J. Fogarty, Geometric Invariant Theory, 2nd ed., Springer-Verlag, New York, 1982.

[R] M. Rapoport, letter to M. Goresky and R. MacPherson, 1991.

[Sa] L. Saper, Tilings and finite energy retractions of locally symmetric spaces, Comment. Math. Helv. 72 (1997), 167-202.

[S1] I. Satake, On compactifications of the quotient spaces for arithmetically defined discontinuous groups, Ann of Math. 71 (1960), 555-580.

[S2] Algebraic Structures of Symmetric Domains, Princeton Univ. Press, Princeton, NJ, 1980.

[T] Y.-S. Tai, Peirce domains, Canad. Math. Bull. 42 (1999), 412-416.

[Z1] S. Zucker, $L^{2}$ cohomology of warped products and arithmetic groups, Invent. Math. 70 (1982), 169-218.

[Z2] Satake compactifications, Comment. Math. Helv. 58 (1983), 312-343. 